\title{
THE DESIGN, MANUFACTURE, AND TESTING OF A NOVEL ADHESION SYSTEM FOR A CLIMBING VEHICLE
}

\author{
A Thesis \\ presented to \\ the Faculty of California Polytechnic State University, \\ San Luis Obispo
}

In Partial Fulfillment

of the Requirements for the Degree

Master of Science in Mechanical Engineering

by

Michael William Schier

June 2017 
(C) 2017

Michael William Schier

ALL RIGHTS RESERVED 


\section{COMMITTEE MEMBERSHIP}

TITLE: $\quad$ The Design, Manufacture, and Testing of a Novel Adhesion System for a Climbing Vehicle

AUTHOR: $\quad$ Michael William Schier

DATE SUBMITTED: June 2017

COMMITTEE CHAIR: $\quad$ Thomas Mackin, Ph.D.

Professor of Mechanical Engineering

COMMITTEE MEMBER: $\quad$ Thomas Mase, Ph.D.

Professor of Mechanical Engineering

COMMITTEE MEMBER: $\quad$ Saeed Niku, Ph.D.

Professor of Mechanical Engineering 


\begin{abstract}
The Design, Manufacture, and Testing of a Novel Adhesion System for a Climbing Vehicle Michael William Schier
\end{abstract}

We present the design and fabrication of a prototype wall-climbing vehicle employing a unique combined locomotion and adhesion system in which the adhesive vacuum is transmitted through moving, perforated treads. Implementing the adhesion/drive system involved a broad range of design challenges, including: developing reliable sealing of sliding and static interfaces, understanding the frictional interactions between the drive treads and various vehicle components and surfaces on which they ride, as well as designing for lightness, manufacturability, and adjustability. The clean sheet design presented in this thesis was taken from concept to functioning prototype in less than 6 months, requiring a considered mix of off-the-shelf components, custom fabrication, and outsourced production. Proof of concept testing is reviewed, including static pressure and force results as well as dynamic vertical surface maneuverability trials. 


\section{ACKNOWLEDGMENTS}

I would like to extend my thanks and appreciation to Dr. Thomas Mackin for providing invaluable support, advice, and good conversation though all stages of this project. Thanks to the numerous professors, mentors, and club advisors at Cal Poly who instilled in me such a deep appreciation for the art of design. Thanks as well to my family for their unwavering patience as I worked my way through too many extra years of higher education, and to my friends and classmates for getting through it all with me. 


\section{TABLE OF CONTENTS}

Page

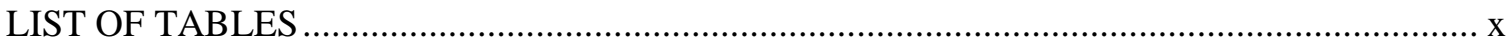

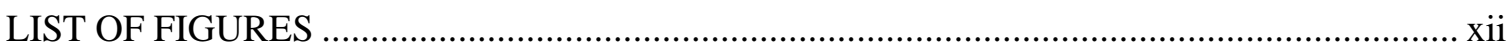

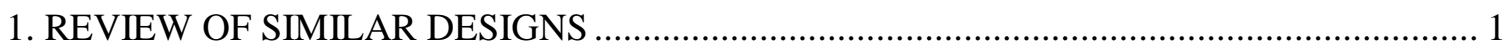

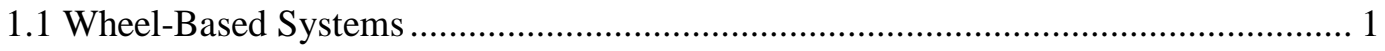

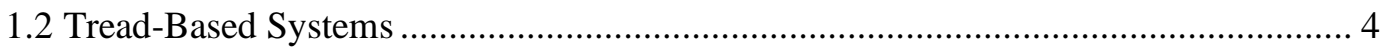

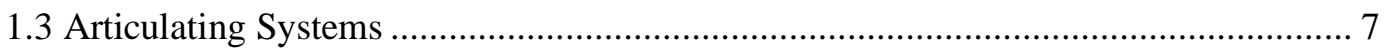

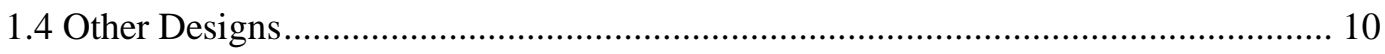

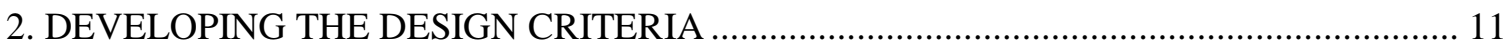

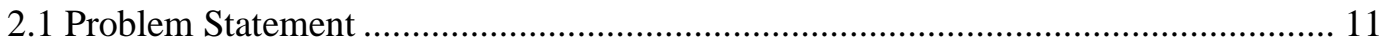

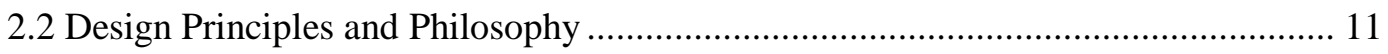

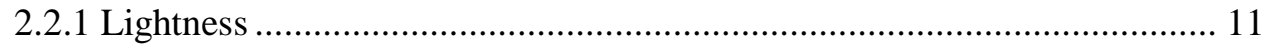

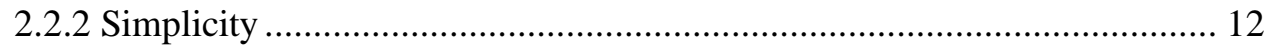

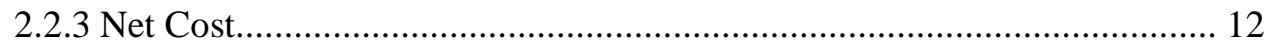

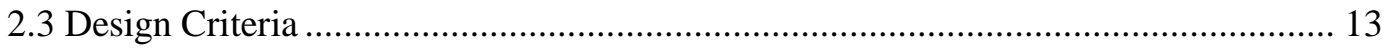

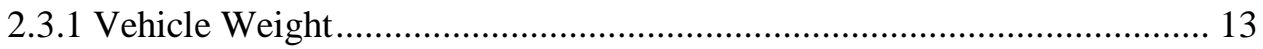

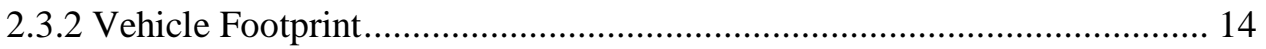

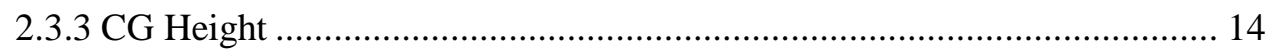

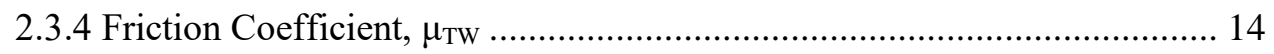

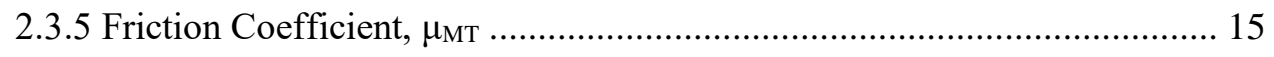

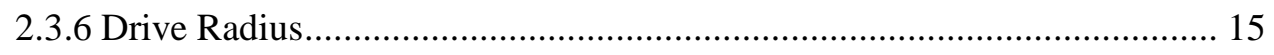

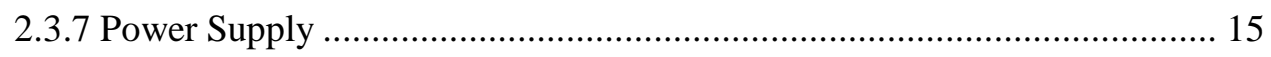

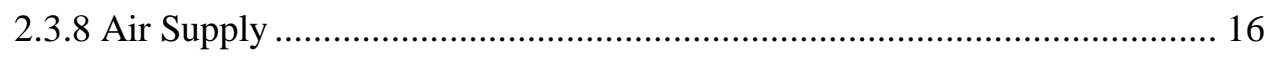

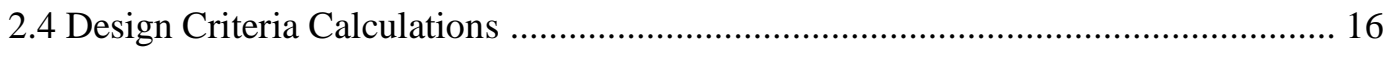




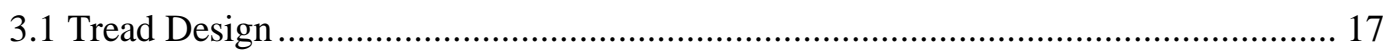

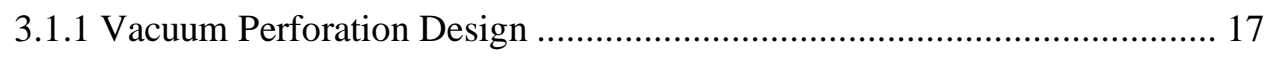

3.1.2 Tread Base Material Selection ............................................................. 19

3.1.3 Tread Backing Material Selection ....................................................... 20

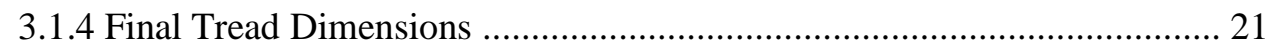

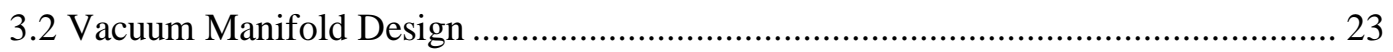

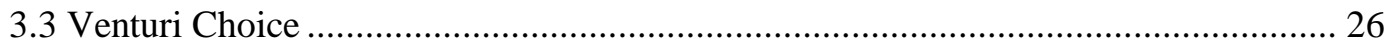

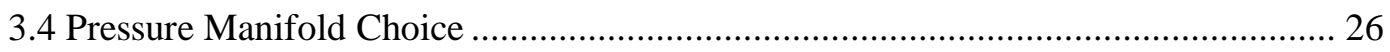

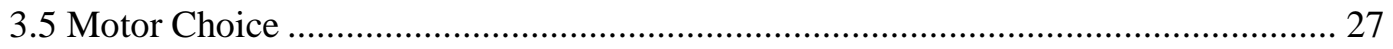

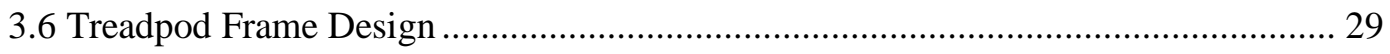

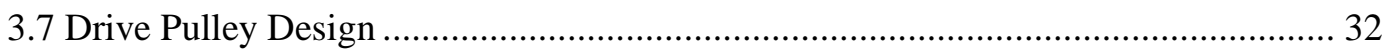

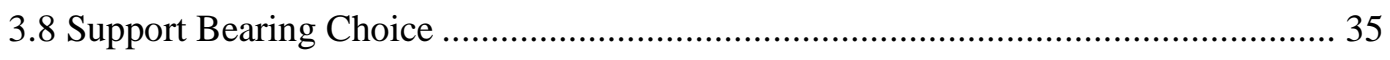

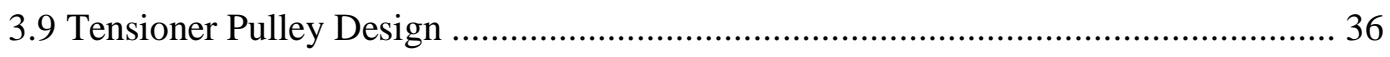

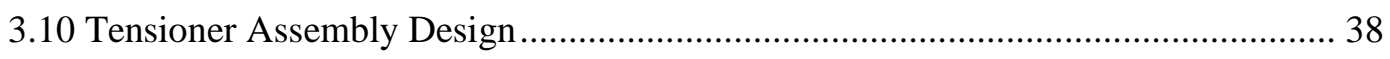

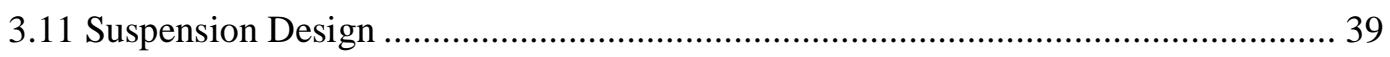

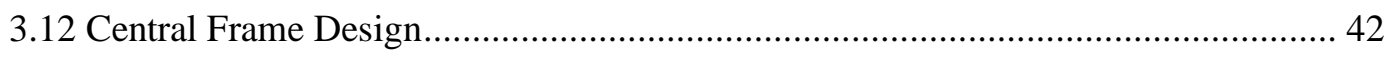

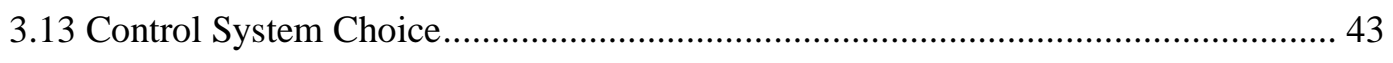

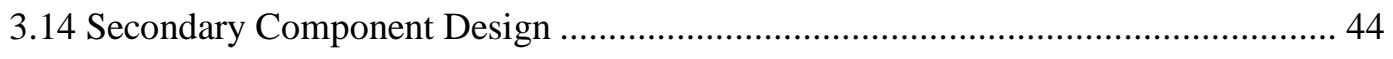

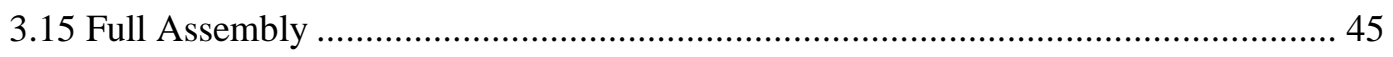

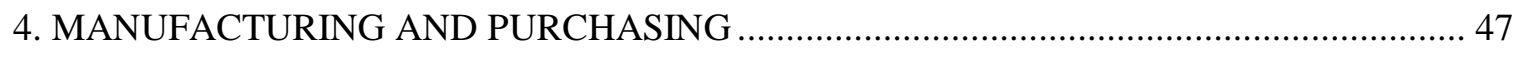

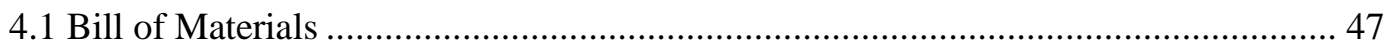

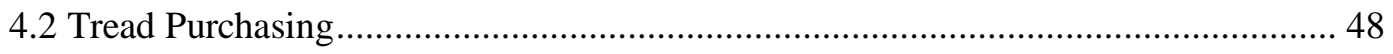

4.3 Vacuum Manifold Manufacturing ................................................................. 50

4.4 Motor Subassembly Manufacturing …........................................................... 52

4.5 Drive and Tensioner Pulley Manufacturing .......................................................... 52 


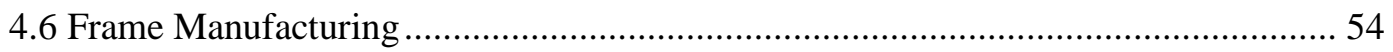

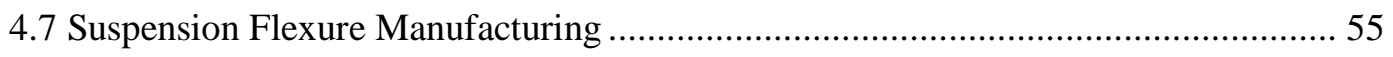

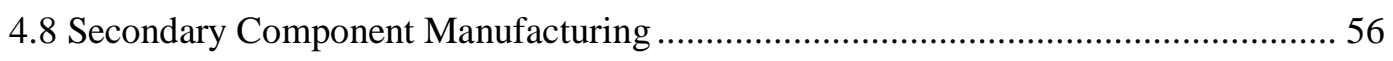

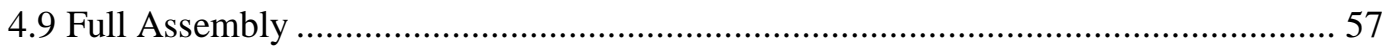

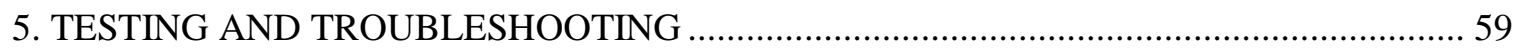

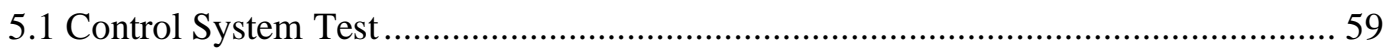

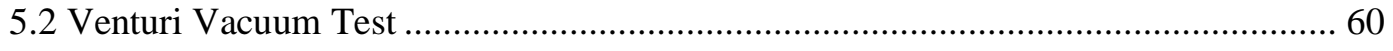

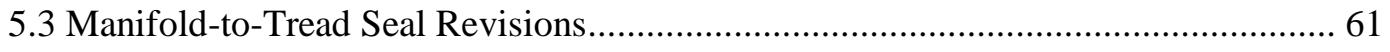

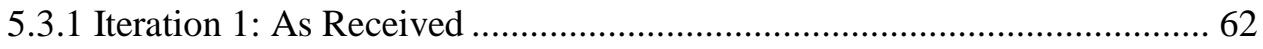

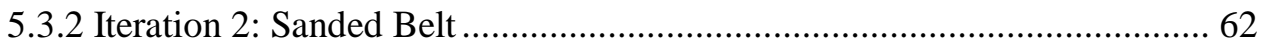

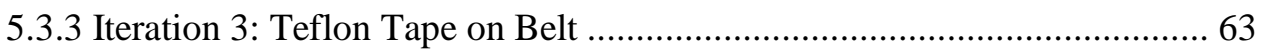

5.3.4 Iteration 4: Cast Silicone Caulking Manifold Seal.................................. 64

5.3.5 Iteration 5: Silicone Rubber Manifold Seal ......................................... 65

5.3.6 Iteration 6: EPDM Foam Manifold Seal .............................................. 66

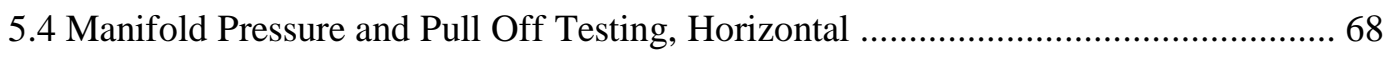

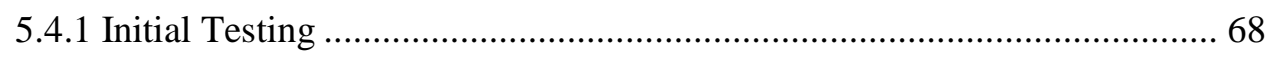

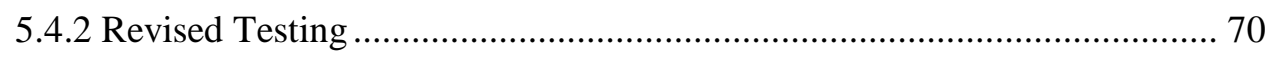

5.5 Vertical Maneuvering Testing on Multiple Surfaces ............................................ 74

5.5.1 Glass Surface Testing ................................................................... 74

5.5.2 Painted Concrete and Painted Plywood ............................................... 76

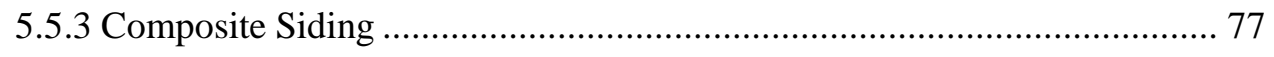

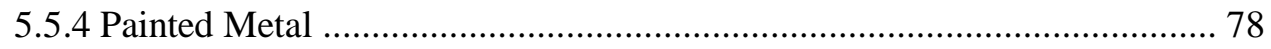

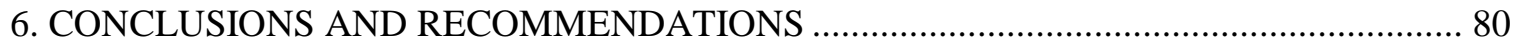

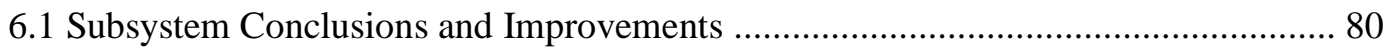

6.1.1 Tread Perforation Design ................................................................ 80

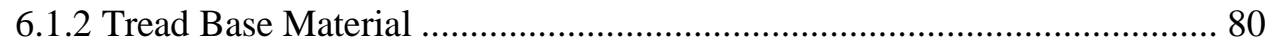




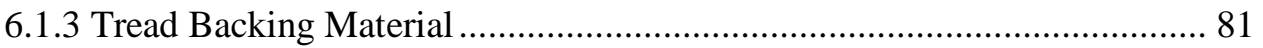

6.1.4 Vacuum Manifold and Manifold Seal .................................................... 81

6.1.5 Venturi Pumps and Pressure Manifold ................................................. 82

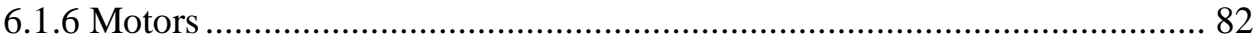

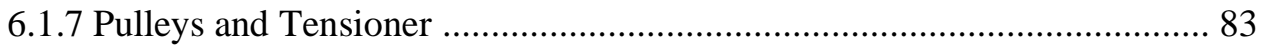

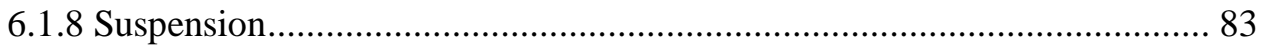

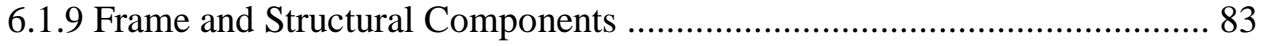

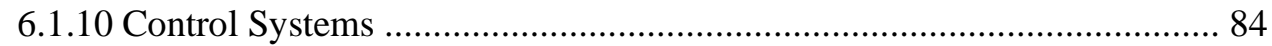

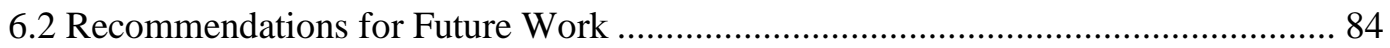

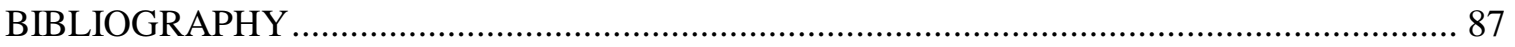

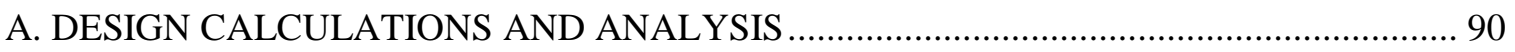

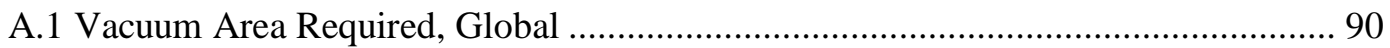

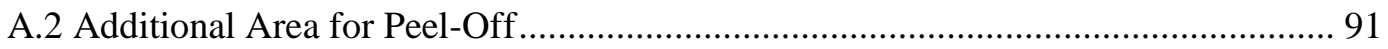

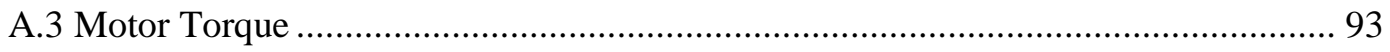

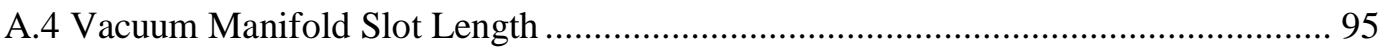

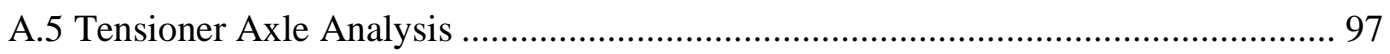

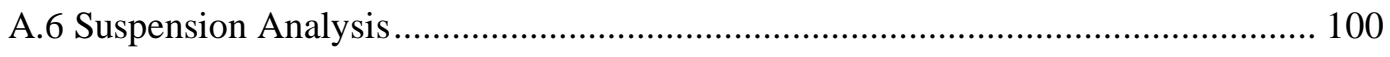

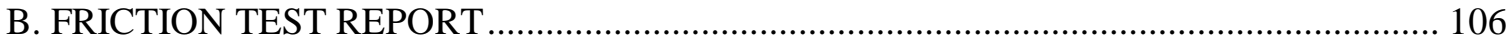




\section{LIST OF TABLES}

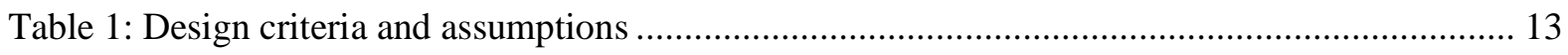

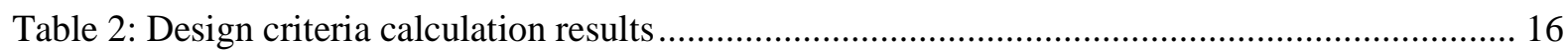

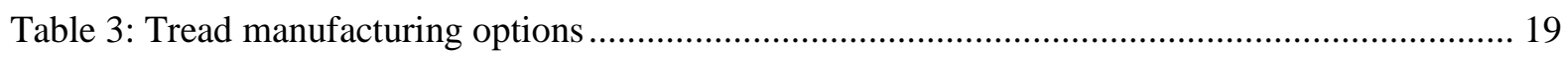

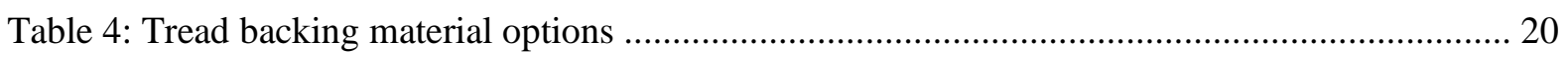

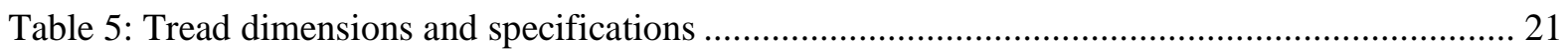

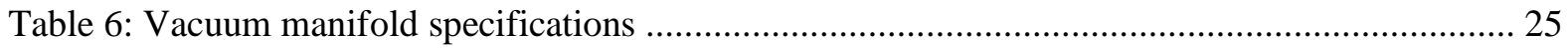

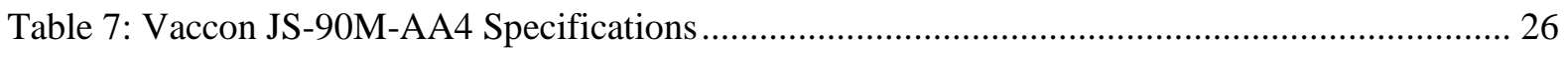

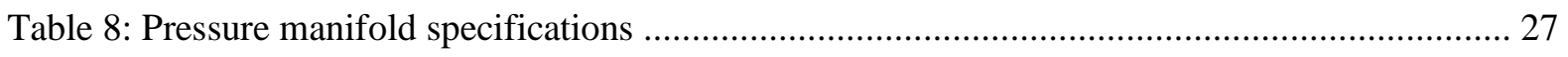

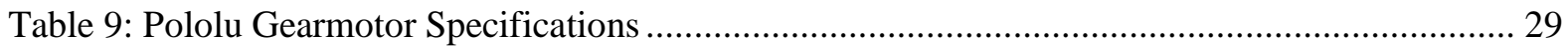

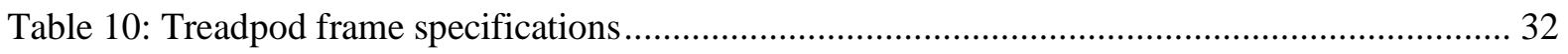

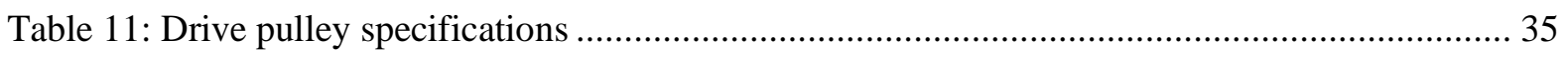

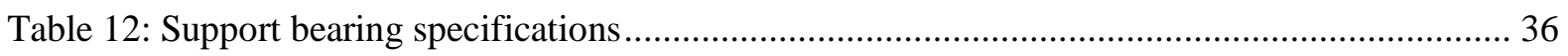

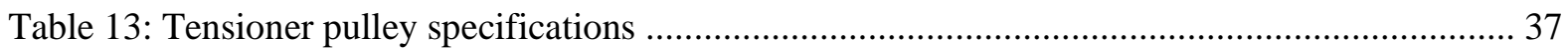

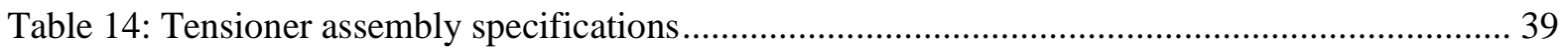

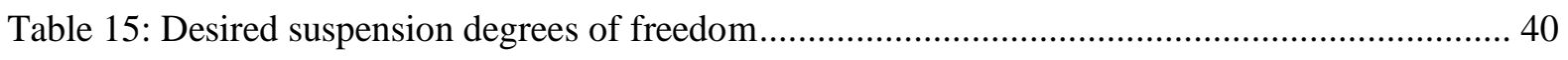

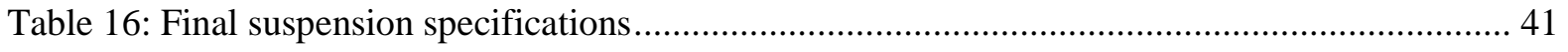

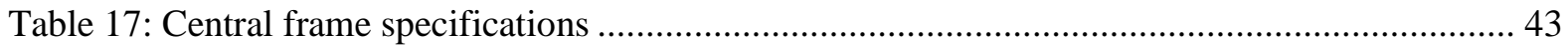

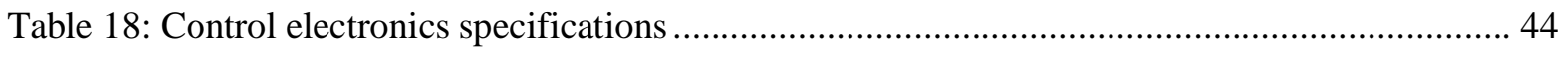

Table 19: Secondary component specifications …................................................................. 45

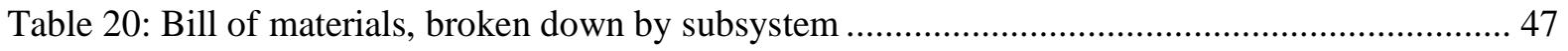

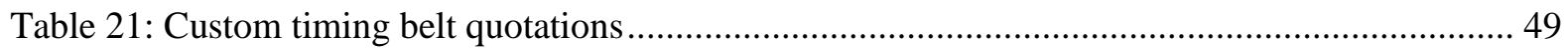

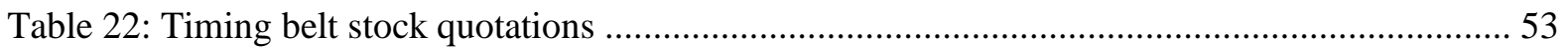




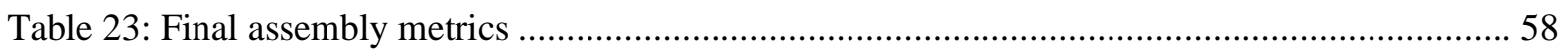

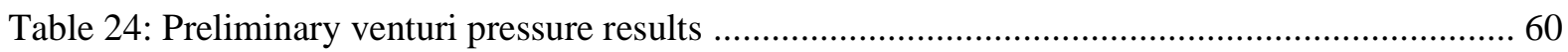

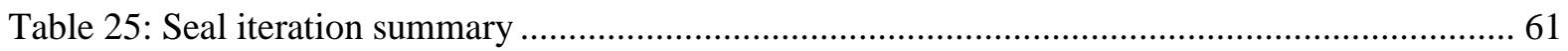

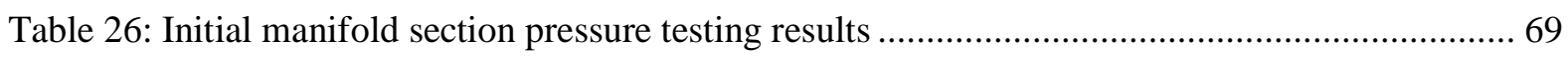

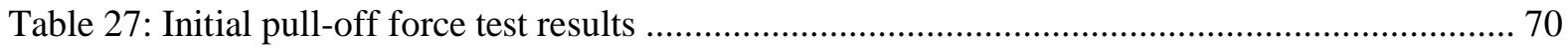

Table 28: Final manifold section pressure testing results ........................................................... 73

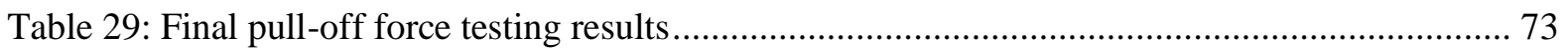

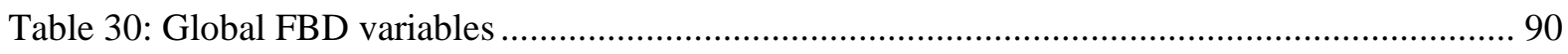

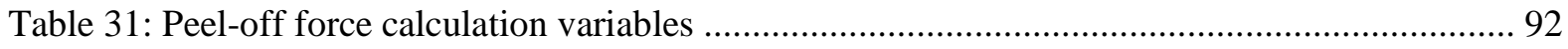

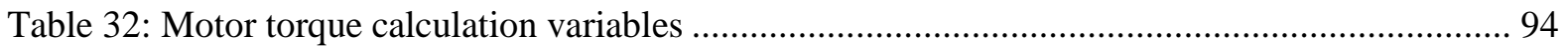

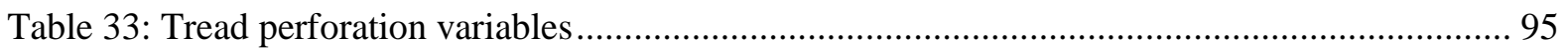

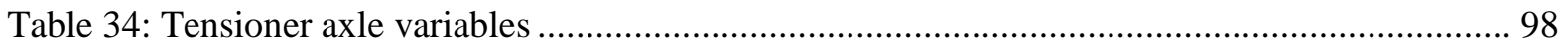

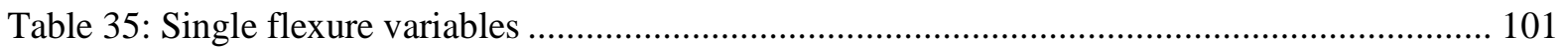

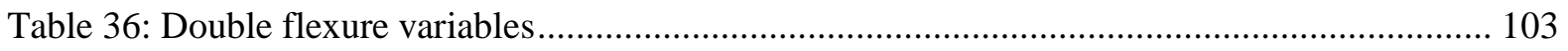

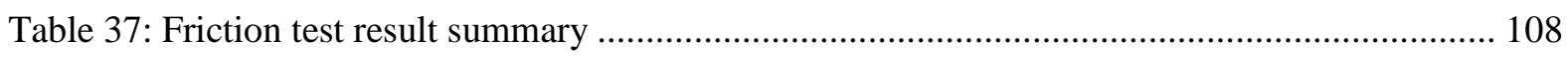

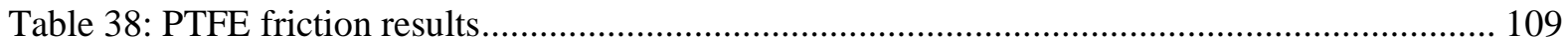

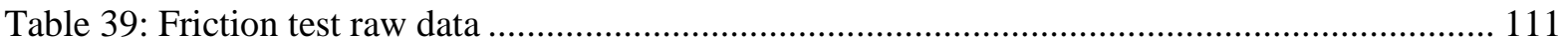




\section{LIST OF FIGURES}

Page

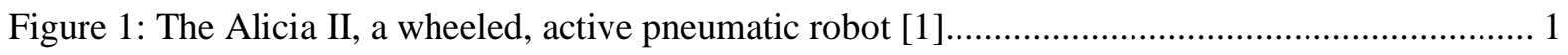

Figure 2: A wheeled robot utilizing the Bernoulli effect [2] ....................................................... 2

Figure 3: TheVertiGo, a wheeled robot using directed thrust [3] ................................................. 3

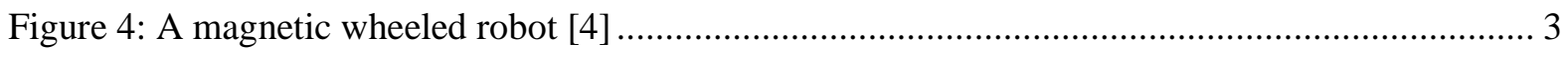

Figure 5: Multi-sectioned magnetic wheeled climber [5] .......................................................... 4

Figure 6: A treaded robot utilizing permanent magnets [6] ........................................................ 5

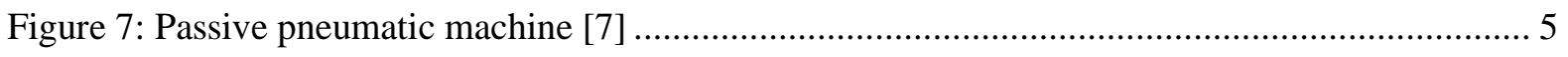

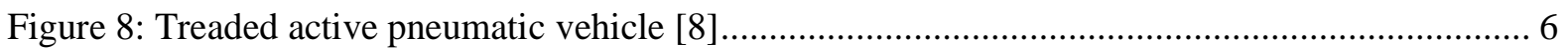

Figure 9: Treaded vehicle with central active pneumatic system [9] ............................................ 6

Figure 10: Small vehicle based on electrostatic adhesion [10] ................................................ 7

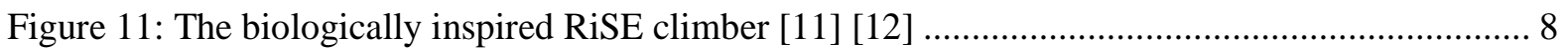

Figure 12: "Treebot" clawed climber [13] …....................................................................... 9

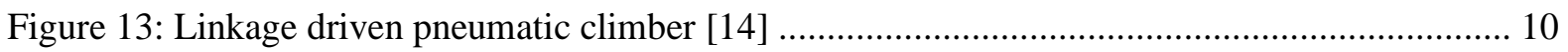

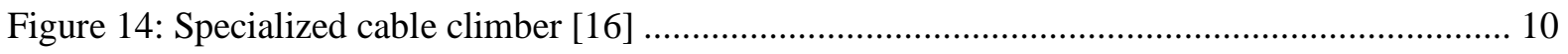

Figure 15: Three comparable tread perforation designs............................................................ 18

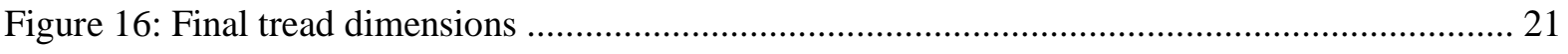

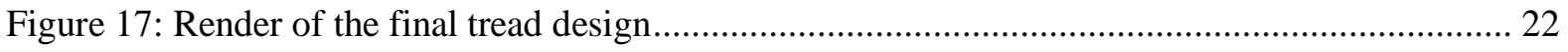

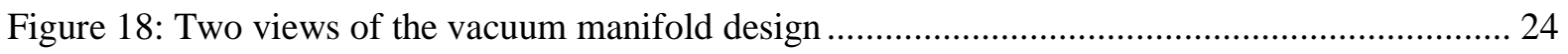

Figure 19: Detail views of the short, bottoming NPT tapped holes in the manifold ......................... 25

Figure 20: Render of the Vaccon JS-90M-AA4 venturi pump ….................................................. 26

Figure 21: Render of the SMC Pneumatics KM11-07-11-10 …................................................. 27

Figure 22: Render of the Pololu 131:1 37D Metal Gearmotor..................................................... 28 
Figure 23: Render of a single treadpod.

Figure 24: Renders of both frame configurations, motor-side (top) and outboard (bottom) .............. 30

Figure 25: Cutaway render of a treadpod, showing internal structure .......................................... 31

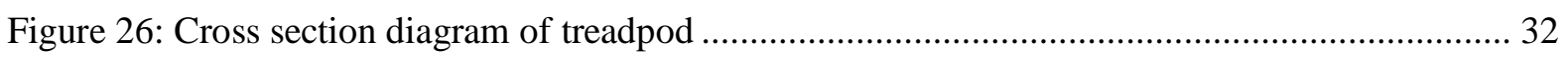

Figure 27: Chart of minimum pulley diameters provided by F.N. Sheppard [21] ........................... 33

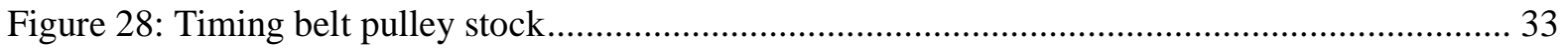

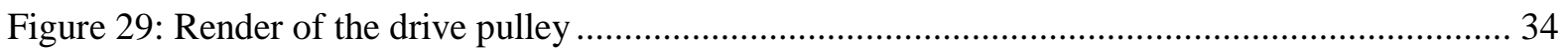

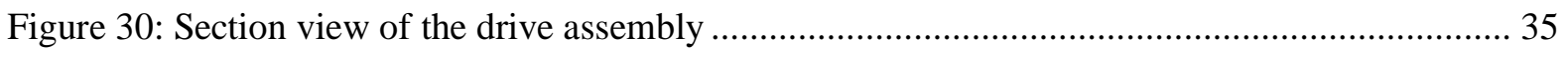

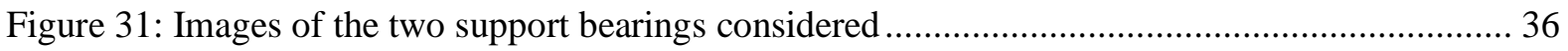

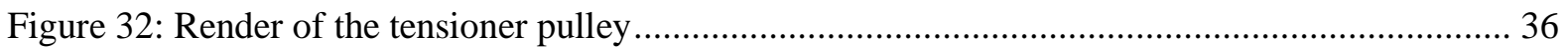

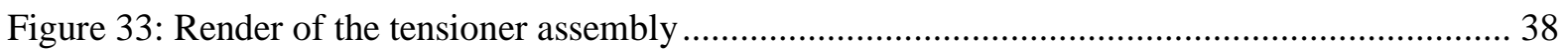

Figure 34: Full assembly render with overlaid coordinate axes ................................................. 39

Figure 35: The preliminary (left) and final (right) suspension flexure designs ............................... 41

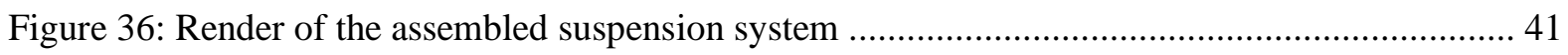

Figure 37: The central frame, bare (left) and assembled (right) ................................................ 42

Figure 38: The speed controller, receiver, and transmitter used in the control system..................... 43

Figure 39: Renders of the secondary machined components ...................................................... 44

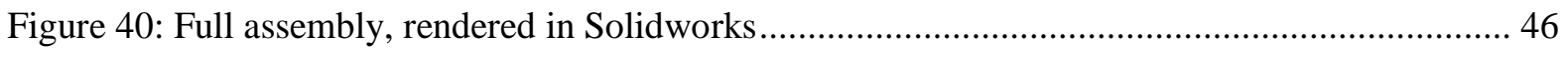

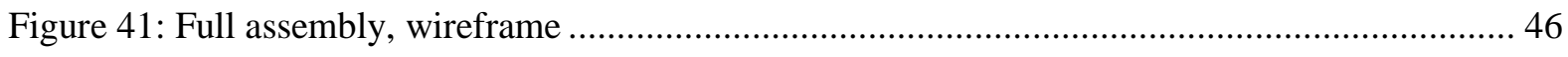

Figure 42: In-progress images of the belts provided by F.N. Sheppard ...................................... 49

Figure 43: Top view of the vacuum manifold, assembled ........................................................ 50

Figure 44: Bottom view of the vacuum manifold, assembled .................................................... 51

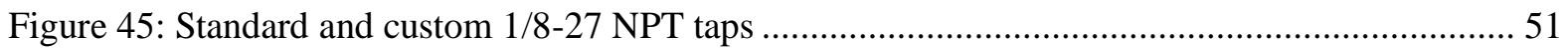

Figure 46: Assembled and soldered motor system, one side ..................................................... 52 
Figure 47: Remaining pulley stock with the machined parts for one treadpod

Figure 48: Nested parts ready for waterjet on a 12"x24" sheet ................................................... 54

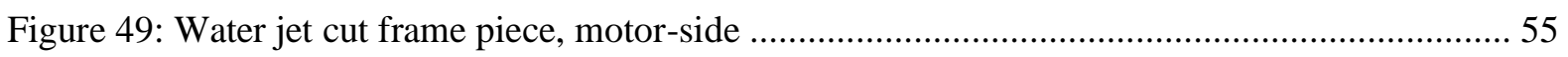

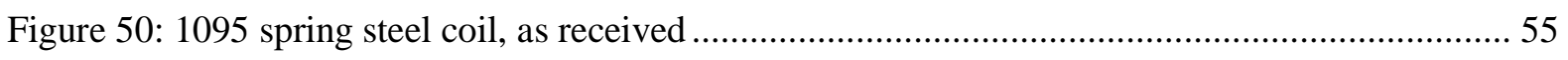

Figure 51: All machined parts for one treadpod ….............................................................. 56

Figure 52: Photograph of the vehicle when first assembled ....................................................... 57

Figure 53: Full assembly, venturis and manifold sections numbered ......................................... 61

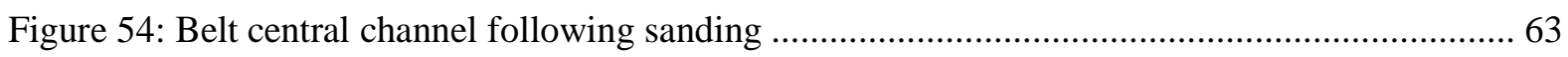

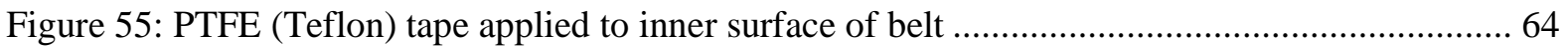

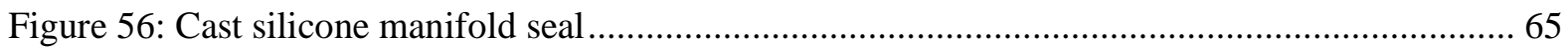

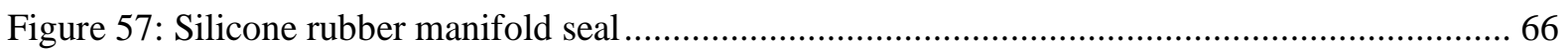

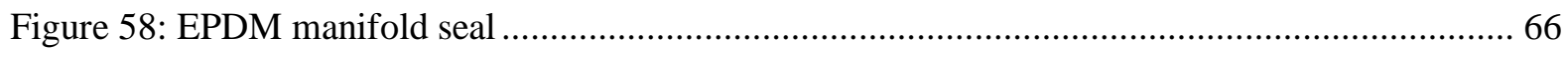

Figure 59: Still from video showing the first successful climbing test on adjustable glass surface .... 67

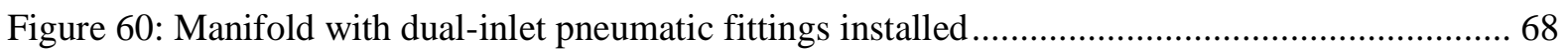

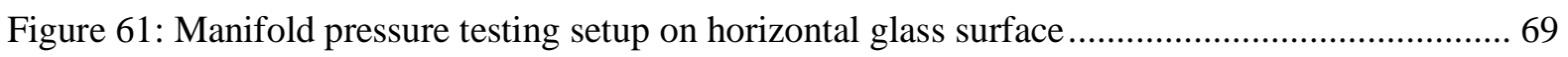

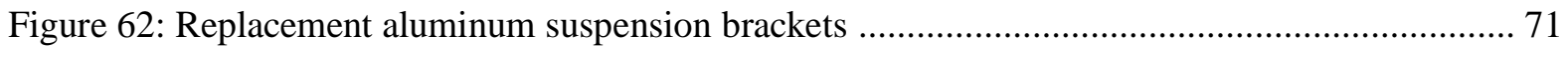

Figure 63: Manifold height excessive, causing gaps at either end ............................................ 71

Figure 64: Manifod height properly adjusted, creating flush sealing surface .................................. 72

Figure 65: Damaged PTFE tape due to loose drive pulley set screws........................................... 72

Figure 66: Video frame, horizontal traverse on glass ................................................................ 74

Figure 67: Video frame, vertical climb on glass ................................................................... 75

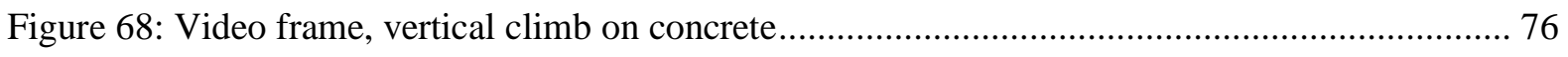

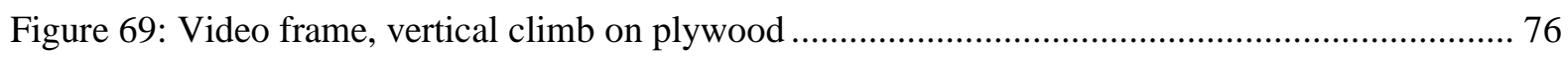

Figure 70: Video frame, horizontal traverse on composite siding .............................................. 77 


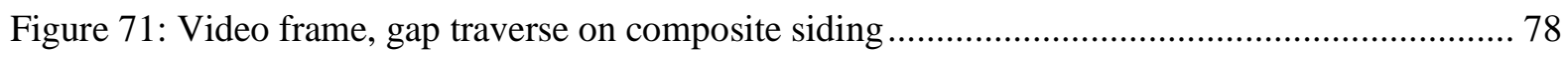

Figure 72: Video frame, final horizontal traverse in square route .............................................. 79

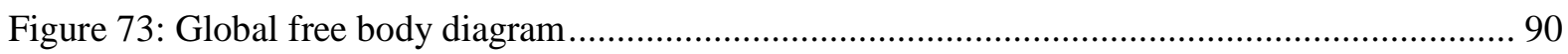

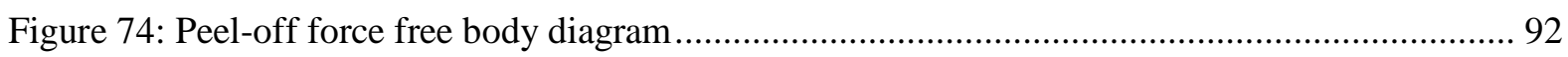

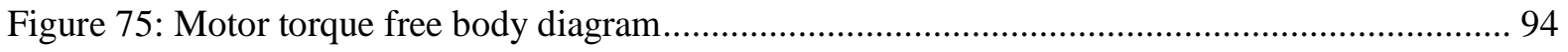

Figure 76: Tread perforation area diagram …........................................................................ 95

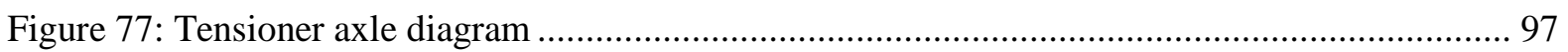

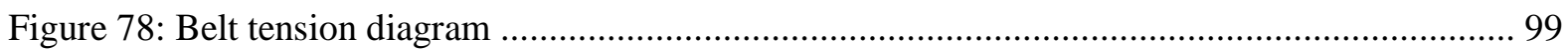

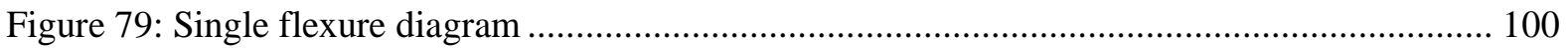

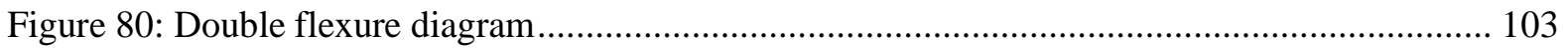

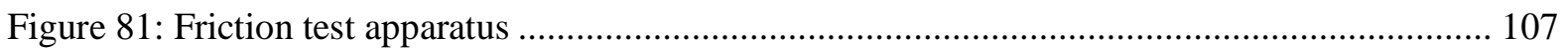

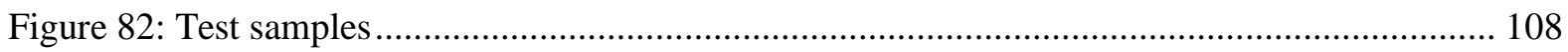

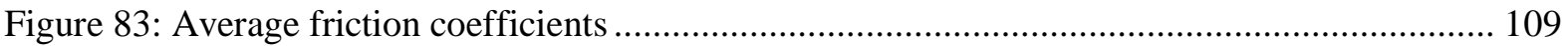




\section{REVIEW OF SIMILAR DESIGNS}

Wall climbing vehicles and robots currently occupy a niche but critical market in industry. Many design firms have released robots to fill a wide range of roles and requirements. The vehicles are often designed to operate in environments that would be hazardous to human workers, such as the interiors of nuclear reactor reservoirs or the exteriors of high-rise skyscrapers, and are designated to perform tasks similar to standard automated systems, but with the added difficulty of performing unsupported on a vertical surface. These tasks can include nondestructive inspection with either cameras or sophisticated sensors, surface preparation and cleaning, including mechanical abrasion, media blasting, or chemical cleaning, and machining processes such as drilling and slotting, Regardless of the objective, the engineering leadership behind each design must decide on both an adhesion method and a locomotion system. Some of the more popular methods are outlined in the following sections.

\subsection{Wheel-Based Systems}

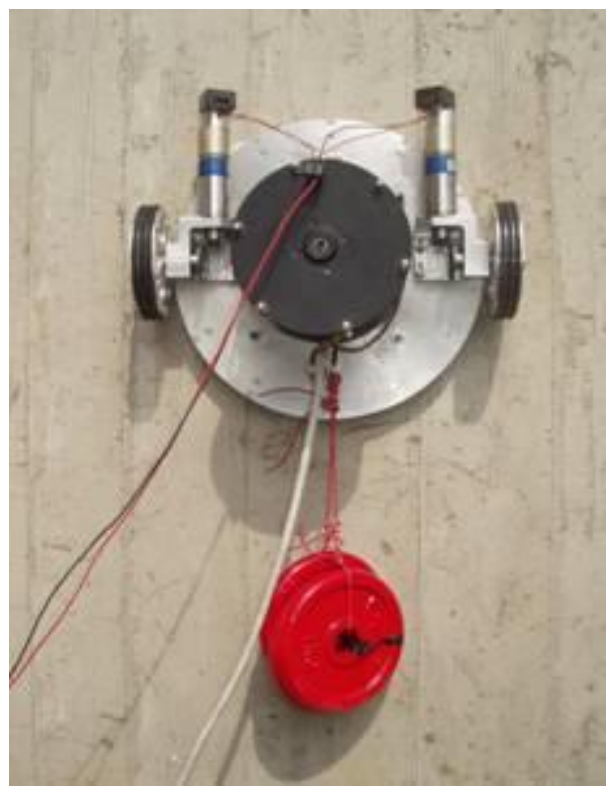

Figure 1: The Alicia II, a wheeled, active pneumatic robot [1] 
One of the most common means of locomotion on climbing vehicles is a wheeled system. This is especially true for many prototype vehicles, as the simplicity of a motor and wheel combination pairs well with many different adhesion methods. For example, the Alice II vehicle in Figure 1 uses a large, central vacuum system to adhere itself to the wall, while powered wheels provide the propulsion required for navigation. Figure 2 demonstrates another interesting concept developed by the University of Canterbury, using pressurized air to create a supersonic air layer underneath two metal platens, allowing the vehicle to hold itself to the wall using the Bernoulli effect. This effect states that fluid traveling faster will be at a lower pressure. Thus, the vehicle uses those pressure effects to produce a non-contact adhesive force.

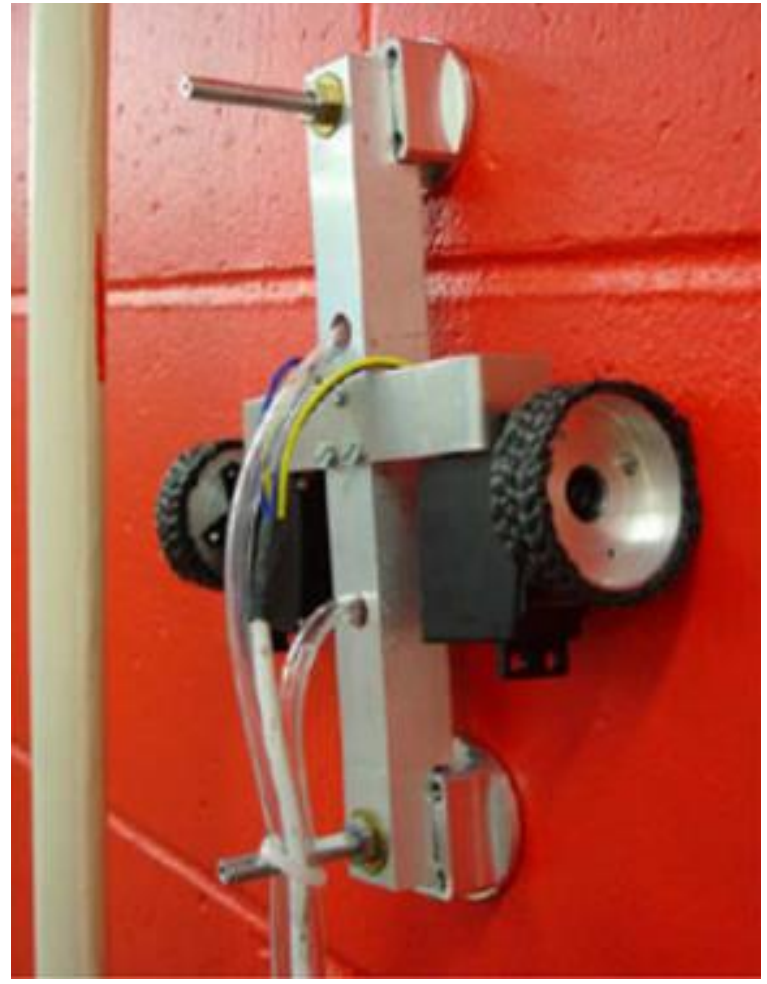

Figure 2: A wheeled robot utilizing the Bernoulli effect [2]

Another wheeled vehicle with a unique adhesion method is Disney Research's VertiGo vehicle. While it is equipped with four steerable wheels, two directionally adjustable propellers built into the central frame exert all propulsive or adhesive forces on the vehicle, from wall 
adhesion to movement thrust. Thus, the wheels are not powered, but simply provide friction against horizontal or vertical running surfaces to assist in maneuvering. These few examples show the diversity of uses of wheels on climbing vehicles.

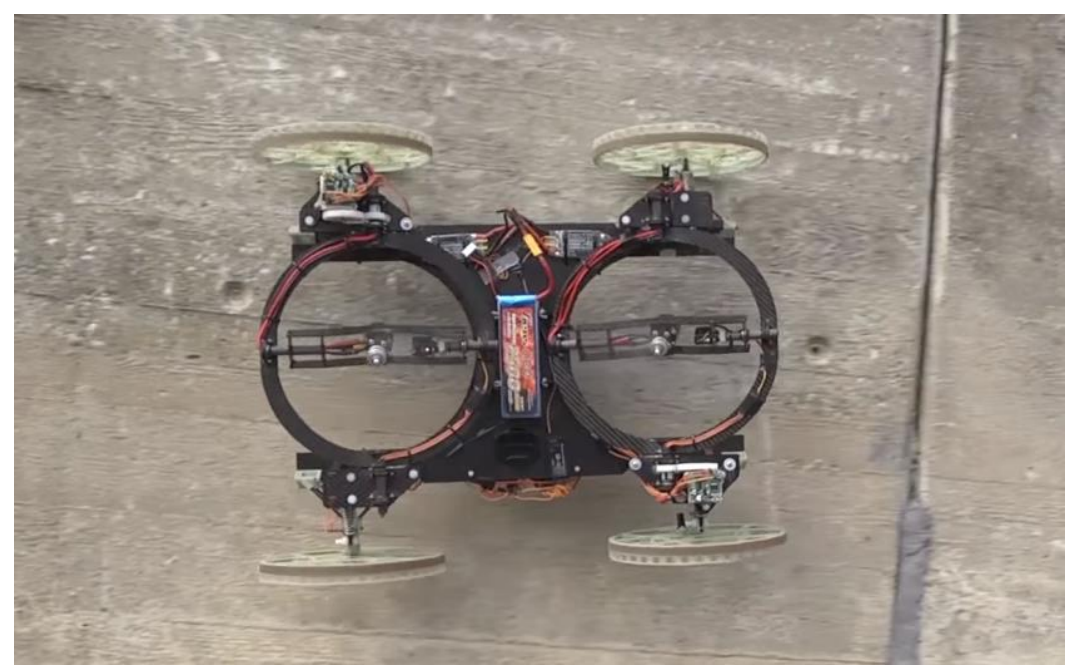

Figure 3: TheVertiGo, a wheeled robot using directed thrust [3]

None of the vehicles above, however, integrated their adhesion methods into the wheels themselves. Because of the limited contact patch of a round disk on a flat surface, most adhesion methods which require surface contact are not feasible for this class of vehicle. There are several exceptions to this generalization, and magnetic adhesion is the most common.

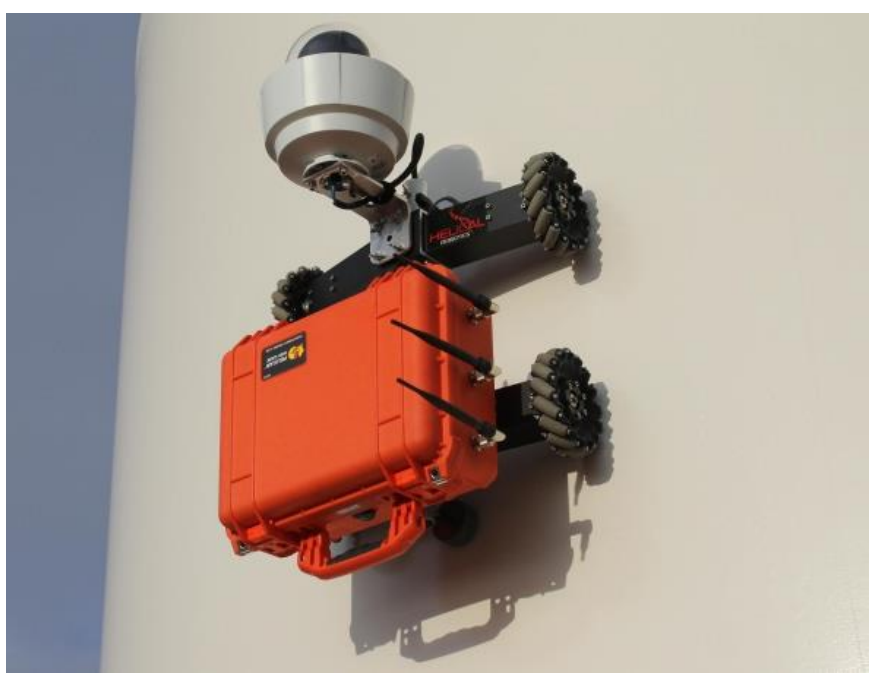

Figure 4: A magnetic wheeled robot [4] 
A wide range of vehicles using magnetic adhesion on the drive wheels are on the market, thanks to the ease of achieving high magnetic forces when paired with ferrous surfaces. Figure 4 shows a vehicle designed for inspection of wind turbine towers. Using helical wheels with many rubber-coated magnets, the vehicle can maneuver easily on ferrous surfaces.
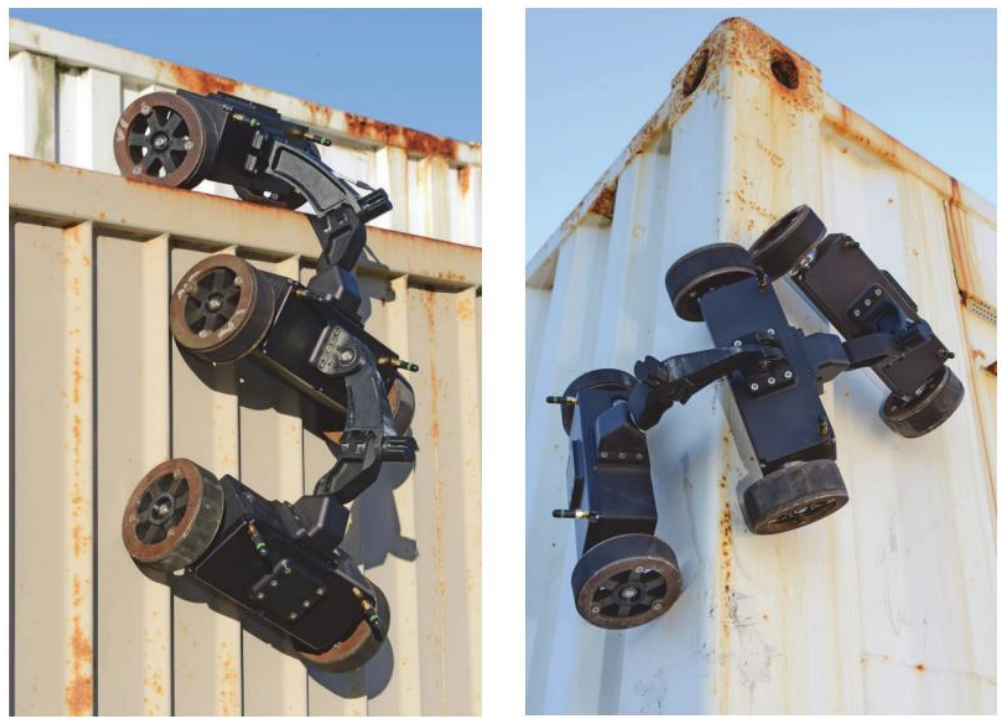

Figure 5: Multi-sectioned magnetic wheeled climber [5]

Similarly, Figure 5 illustrates a complicated, sectioned vehicle using magnetic wheels to climb the sides of steel shipping containers. While the control systems required for this vehicle are sophisticated, the adhesion method is simple and straightforward. The obvious disadvantage of such systems is their inability to function on any surface that is not magnetically attractive.

\subsection{Tread-Based Systems}

Another popular and common locomotion method are tank treads. Inherently providing a large tread area in contact with the surface being climbed, many designers have integrated adhesion methods onto the drive treads themselves. Figure 6 illustrates the simple concept of 
attaching permanent magnets to the drive belts to allow for adhesion and locomotion on ferrous surfaces.

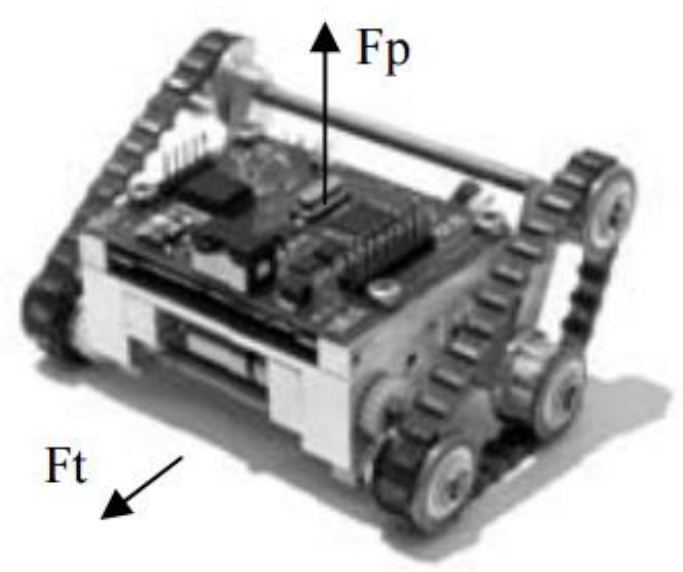

Figure 6: A treaded robot utilizing permanent magnets [6]

Pneumatic adhesion systems are commonly combined with treaded designs as well.

Passive pneumatics, or suction cups, simply rely on the initial generated suction force to hold the vehicle on the wall, as in Figure 7. Most vehicles attempt to avoid the difficulties of a passive system by employing active pneumatic by generating their own vacuum. Figure 8 shows a vehicle using active pneumatics in the form of vacuum cups fed by a rotating valve.

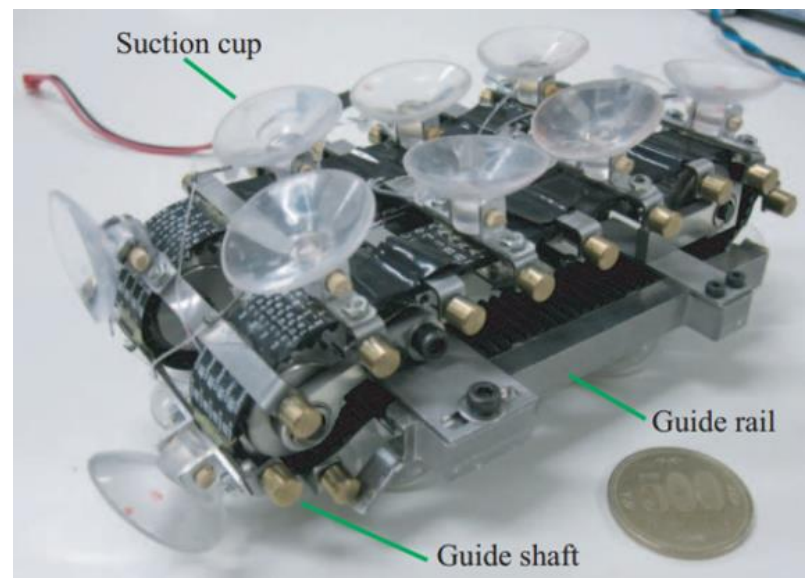

Figure 7: Passive pneumatic machine [7] 


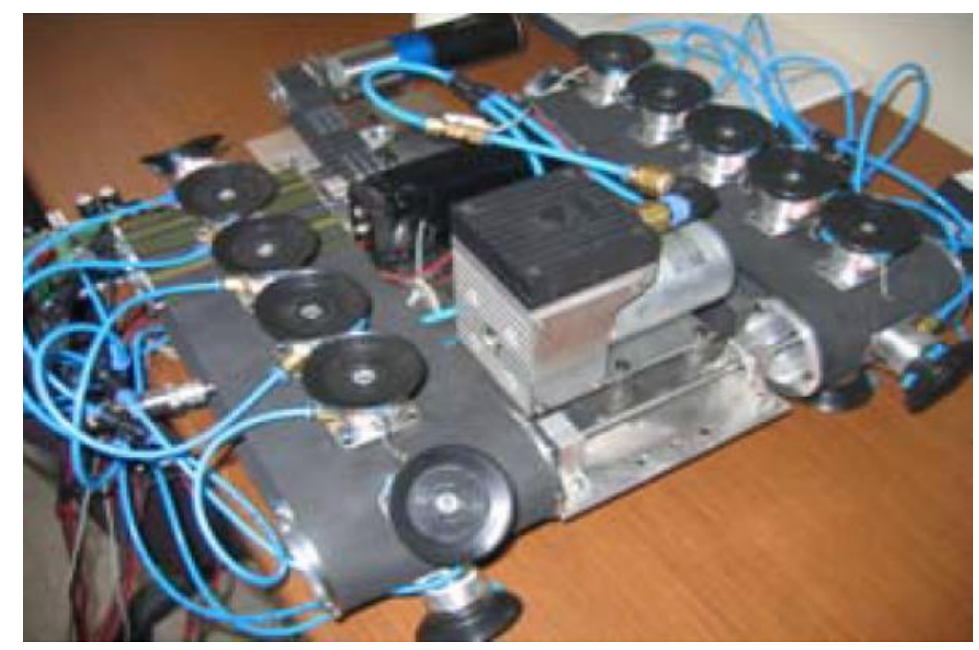

Figure 8: Treaded active pneumatic vehicle [8]

Similar in concept to Figure 1 above, Figure 9 shows a climbing vehicle developed by International Climbing Machines (ICM). ICM's vehicle uses a central active pneumatic system to provide adhesive force and two compliant drive treads to provide propulsion. The unique design outlined in the following report is most similar to these designs, as it will employ a combined active pneumatic system integrated into the drive treads.

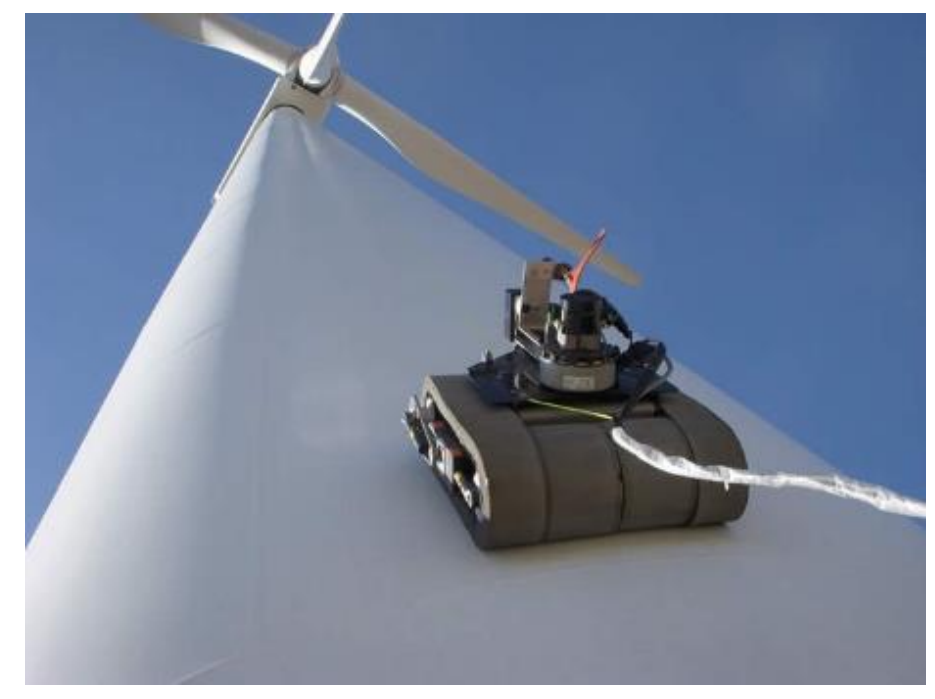

Figure 9: Treaded vehicle with central active pneumatic system [9] 
Several alternative adhesion methods are used on treaded vehicle as well. Figure 10 shows a prototype vehicle utilizing electrostatic adhesion on a moving tread to generate sufficient force to hold the robot to the wall. This is another clear demonstration of the advantage of the large surface area provided by treads, as electrostatic forces are typically very weak unless acting over a large area.

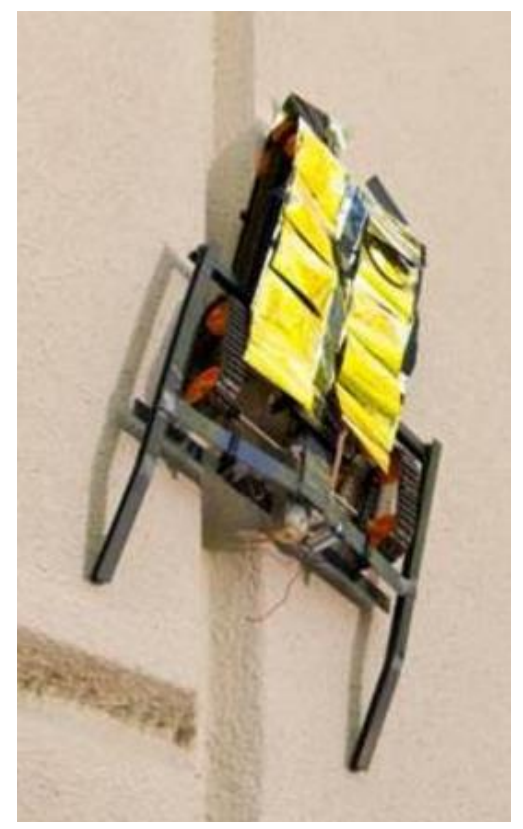

Figure 10: Small vehicle based on electrostatic adhesion [10]

\subsection{Articulating Systems}

The third common style of climbing vehicle design utilizes articulated limbs to grip and manipulate the climbing surface, providing propulsion. The theoretical advantage of this class of climbing vehicle is the ability to adapt to irregular, rough, or rugged terrain. Unlike many of the prototypes outlined above, this capability typically requires sophisticated control systems and many motors and actuators. A prime example of this methodology is the RiSE robot created by Boston Dynamics. Using a hexapodal, biologically inspired design, the RiSE machine uses end effectors with hooks, gecko-like fibrous feet, or rubber feet to climb a variety of surfaces. 


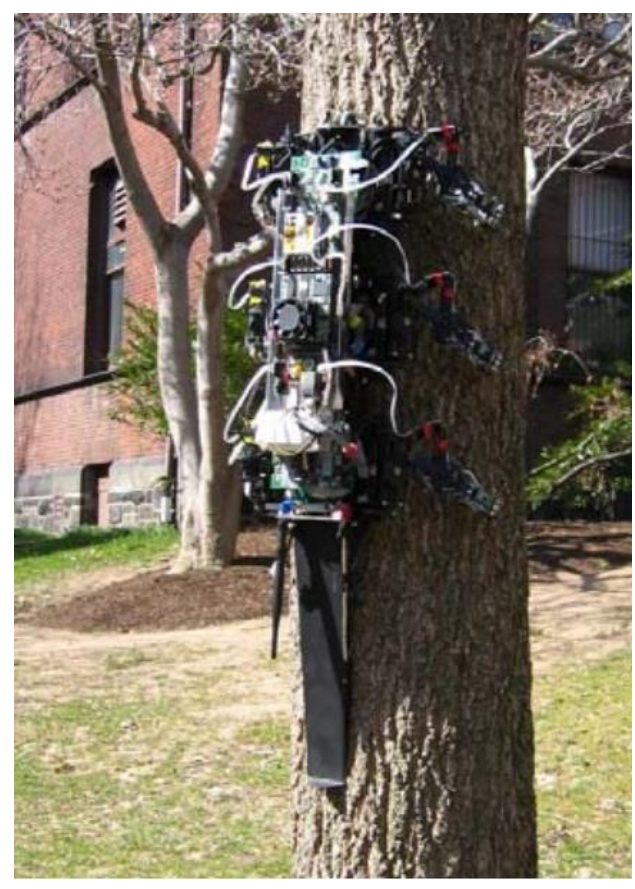

Figure 11: The biologically inspired RiSE climber [11] [12]

This is not to imply that simpler articulated robots are not designed. The Treebot, shown in Figure 12, is an inchworm style robot that uses several motors to extend, retract and actuate two grasping claws in order to hold onto branches. While simpler than RiSE, the Treebot still requires some control feedback from the grippers in order to climb reliably. Additionally, its movement speed is limited, a common problem for articulated robots. 


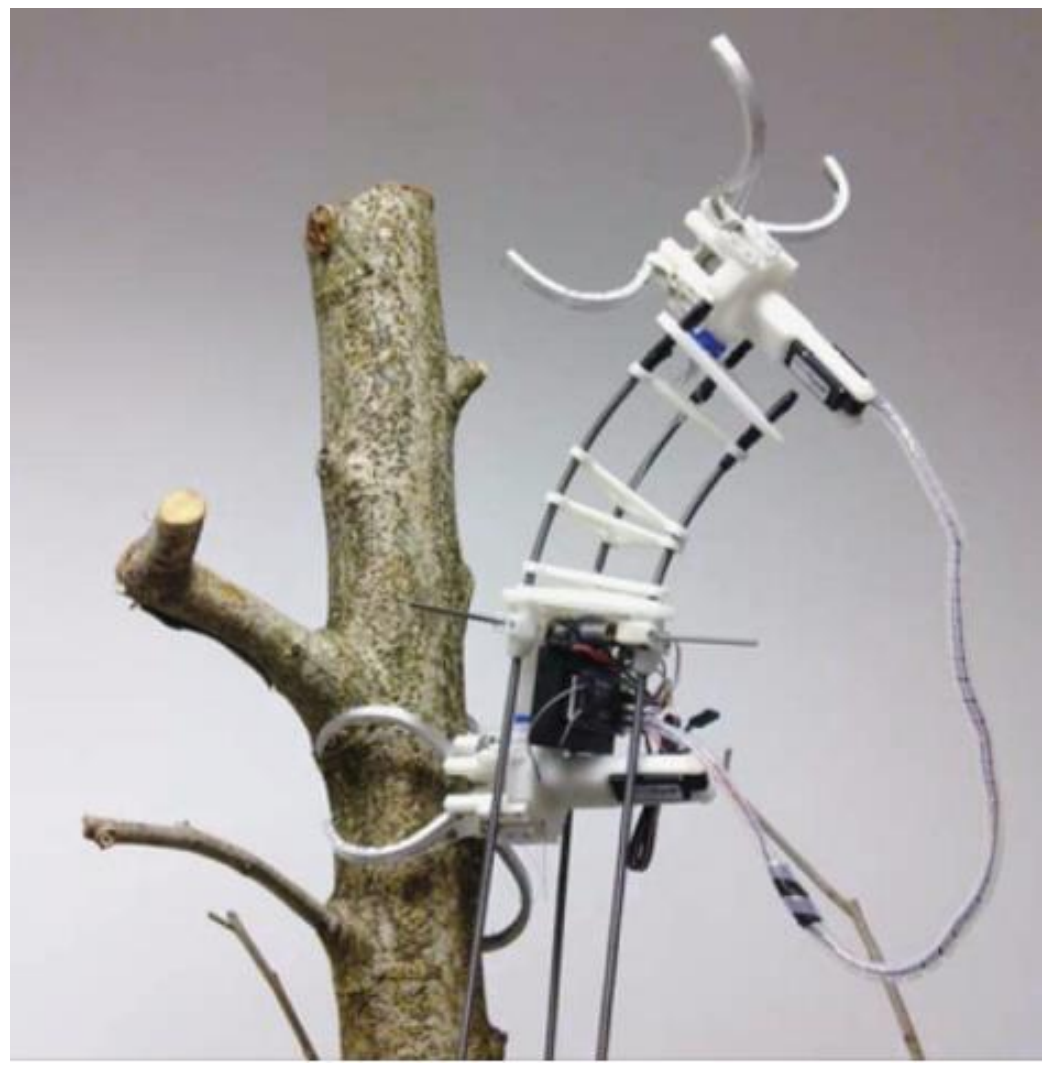

Figure 12: "Treebot" clawed climber [13]

An even simpler articulating design is shown in Figure 13. Using pressurized water to generate vacuum pressure through venturis, actuate switches, power hydraulic actuators, and finally to wash a window, this vehicle uses a simple linkage system to pivot the vacuum feet and climb a glass surface. While an intriguing concept, it lacks the adaptability that is the primary advantage of articulating systems. 


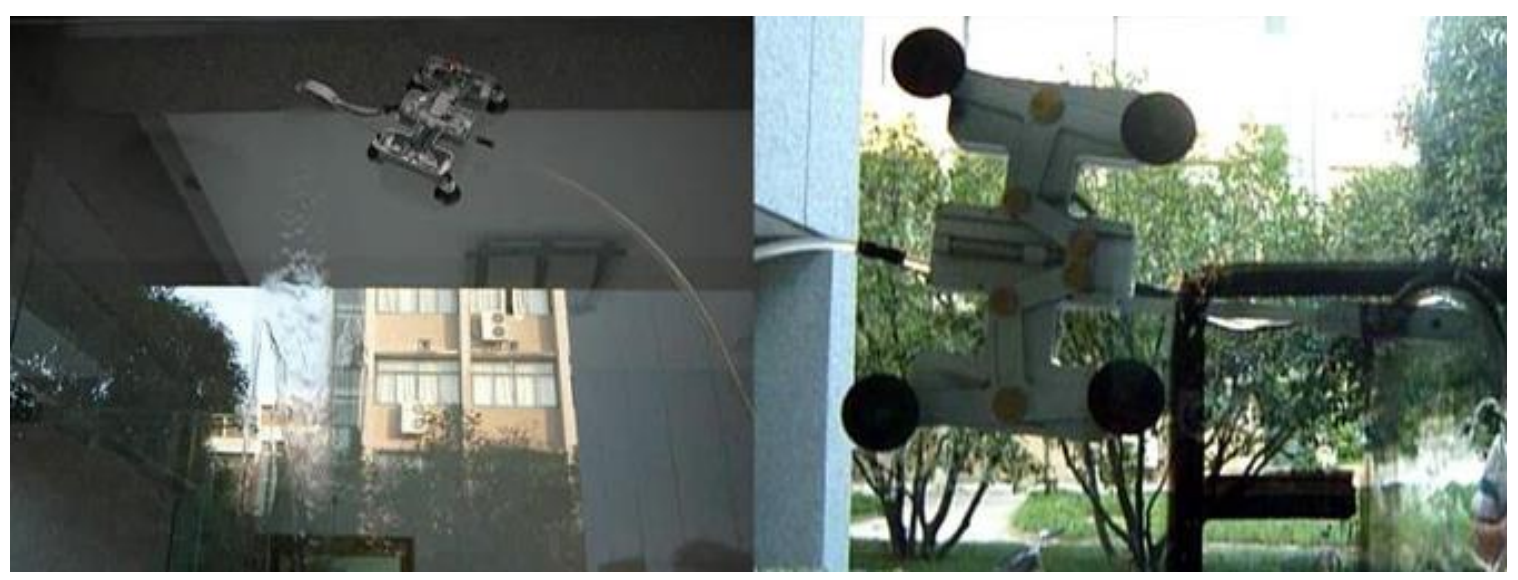

Figure 13: Linkage driven pneumatic climber [14]

\subsection{Other Designs}

There are certainly many other methods to climb a wall. Specialized robots are often designed for a single purpose, such as the cable climber in Figure 14. Sliding frame mechanisms employ two adhesive units that can move relative to each other in order to move, inchworm-style, along a surface. Adhesive or elastomer-based adhesion is gaining traction in research and industry as well. A very wide variety of articulating climbing bots have been built and documented.

Reference the bibliography, and specifically reference [15] for more information.

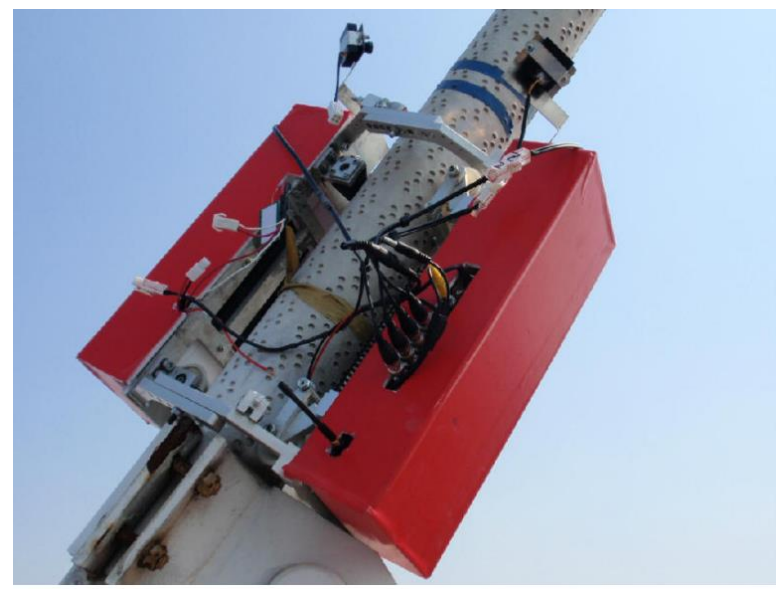

Figure 14: Specialized cable climber [16] 


\section{DEVELOPING THE DESIGN CRITERIA}

\subsection{Problem Statement}

The project goal was to design, build, and test a prototype of a wall climbing vehicle employing a novel adhesion/drive concept and to perform proof-of-concept testing on vertical surfaces demonstrating adhesion and maneuvering ability. By using perforated, moving treads and a static vacuum manifold on the vehicle's body, vacuum can be drawn through the treads in order to adhere the vehicle to the climbing surface. This unique concept differentiates the following design from the other wall climbing robots currently on the market. Using lessons learned from previous design iterations using the novel adhesion method [15], combined with new research and development, a clean sheet design has been created and a prototype built and tested subject to the constraints of a month timeline.

\subsection{Design Principles and Philosophy}

In order to approach the task of designing this vehicle from scratch, a short list of principle design philosophies was used to guide the form and function of every component of the system.

\subsubsection{Lightness}

Vehicle weight is directly and inextricably linked to the force and power required to climb a wall. Previous vehicles were substantially overbuilt. By drastically reducing weight in all non-critical subsystems and performing necessary analysis and research to cut weight from all parts of the system, the vehicle will stand a measurably better chance of ascending and 
maneuvering upon a vertical wall. A clear, simple way to lighten the vehicle is to reduce vehicle size and minimize the physical volume of all parts inside the vehicle.

\subsubsection{Simplicity}

Simplicity in design is a critical element in the creation of novel prototypes. This principle can be related to design for manufacturability, optimization of the combination of offthe-shelf and custom parts, and reduction in assembly size. All parts requiring machining or forming after purchase will be completed personally, and as such, all parts should be simple enough to be made quickly with manual machining tools or two dimensional numerical control tools such as water jet or laser cutters and without requiring time consuming jigs or fixtures. Intelligent part sourcing contributes heavily to simplification as well. If an off-the-shelf part fully or partially matches the required design form or function, and fits the lightness, schedule, and budgetary constraints, there is little reason to sink unnecessary time and effort to create a functionally identical custom part. The principle of simplicity can, lastly, be interpreted as a reduction of the number of parts required. In addition to directly reducing weight, minimizing part count will expedite manufacturing, assembly, integration, and troubleshooting. For example, using adhesive backed cable tie anchors instead of metal brackets, screws, and nuts to secure venturi pumps and electronic equipment to the vehicle reduces the overall part count, the weight of the vehicle, the number of hole features required in the frame, and the assembly time necessary to install or remove components.

\subsubsection{Net Cost}

While designing and prototyping an unproven concept under a tight timetable, reductions in cost allow for more ease in design changes on the fly and provide freedom to adapt to changing design requirements quickly. Additionally, as a self-funded design, the budget available for the 
project is quite limited. Coincidentally, reducing size/weight of the vehicle and decreasing complexity often perform the bulk of the cost reduction. For example, the drive belts used on previous iterations were priced at over $\$ 300$ each, while the newer, smaller, and lighter belts from the revised design, despite a more intricate perforation design, were quoted at $\$ 156$ each.

\subsection{Design Criteria}

Table 1 lists design values used to perform the initial calculations required to size vehicle components. Assumptions were made where necessary, and support for these assumptions is below, in order of appearance in the table.

Table 1: Design criteria and assumptions

\begin{tabular}{lll}
\hline Criteria & Value & Notes \\
\hline Vehicle Weight, $\mathrm{W}_{\mathrm{V}}[\mathrm{lbf}]$ & 7.5 & Planned weight of prototype vehicle \\
Design Weight, $\mathrm{W}_{\mathrm{D}}[\mathrm{lbf}]$ & 15 & System design weight with safety factor \\
Vehicle Footprint [in. x in.] & $12 \times 12$ & Desired maximum vehicle footprint \\
Vehicle CG Height, h [in.] & 3 & Desired maximum CG height, measured from wall \\
Friction Coefficient, $\mu_{\mathrm{TW}}$ & 0.75 & Coefficient between tread backing and wall \\
Friction Coefficient, $\mu_{\mathrm{MT}}$ & 0.4 & Coefficient between tread and vacuum manifold \\
Pressure, Vacuum, $\mathrm{P}_{\mathrm{V}}[\mathrm{psi}]$ & 13 & Operating vacuum pressure of venturi system \\
Drive Radius, R [in.] & 1 & Estimated net radius of pulley incl. tread thickness \\
Power Supply & N/A & Off-vehicle DC supply, capability for onboard battery \\
Vacuum Supply & N/A & Onboard venturi system, off-vehicle air supply \\
\hline
\end{tabular}

\subsubsection{Vehicle Weight}

As discussed above, vehicle weight has a direct influence on the force required to climb. With a preliminary weight survey of potential parts, the planned net weight of the vehicle was estimated to be $7.5 \mathrm{lbf}$. This allows the vehicle to be manageable and easily handled, avoiding the issues that plagued previous attempts with a vehicle weighing twice that amount.

However, all design was based on a design weight of $15 \mathrm{lbf}$. This safety factor of 2 was chosen to provide for several possibilities. The torque required by the motors will include a designed safety buffer to ensure no motor stall. The adhesive force will, similarly, be scaled by a 
factor of two. This theoretically provides capability, given an as-designed $7.5 \mathrm{lbf}$ vehicle, to operate with half of the vehicle's maximum adhesion. This redundancy can come in the form of broken seals on one or several vacuum chambers, which directly reduces vacuum force, or a decrease in the coefficient of friction between the wall and the tread backing material. Finally, while payload capacity is not a design concern for this prototype, the additional weight capability may permit some secondary weight to be added.

\subsubsection{Vehicle Footprint}

Limiting the vehicle footprint provided a guideline to ensure that excess size, and thus excess weight, did not become a problem during the design of the vehicle. Additionally, this value was used to determine the influence of the "peel-off" force which would attempt to pull the upper vacuum manifolds off of a vertical wall. The magnitude of this force is related to the vehicle length and the CG height from the wall.

\subsubsection{CG Height}

Minimizing CG height off of a vertical surface will minimize the aforementioned "peeloff" force. A designed CG height of 3 in. will allow for sufficient flexibility in component placement while still maintaining a sleek overall profile.

\subsubsection{Friction Coefficient, $\mu_{T W}$}

The friction coefficient between the tread backing material and the wall surface was estimated at 0.75 . This conservative estimate was derived from several sources: a simple friction coefficient test performed on backing material samples obtained from the belt supplier, FN Sheppard, and literature from Gates Mectrol, a secondary belt supplier, as well as other sources. 
The test write-up and data can be found in B. FRICTION TEST REPORT, while the reference literature can be found in [17], [18], and [19].

\subsubsection{Friction Coefficient, $\mu_{M T}$}

A bounding, conservative friction coefficient between the vacuum manifold and the tread was estimated to be 0.4 . This was determined through a simple friction test performed using samples of the designed manifold seal material, PTFE, and neoprene belt material provided by F.N. Sheppard. Validation of the results was obtained through literature exploring the friction and wear characteristics of PTFE [20].

\subsubsection{Drive Radius}

The total radius of the drive wheel and tread thickness was estimated at $1 \mathrm{in}$. This provides sufficient clearance for motors and allows for a radius of curvature that does not exceed the recommended minimum pulley diameter based on backing material and thickness, provided in reference [21] and in Figure 27.

\subsubsection{Power Supply}

As initially designed, an off-vehicle power supply will provide the electricity to run motors and electronics on the vehicle. As an umbilical will be required to provide the pressurized air, there is little downside to providing the option of off-board power. The electronics will, however, be chosen and wired so that any $6 \mathrm{~V}-12 \mathrm{~V}$ DC source with sufficient current capability using a standard T-connector will be able to be used. During testing of the prototype, an onboard 7.4V lithium polymer battery provided the entirety of the electrical power required. 


\subsubsection{Air Supply}

Pressurized air at the recommended 80 psi will be supplied to the Vaccon JS-90M venturi pumps through an umbilical from a remote air compressor. At this stage, there is no feasible fully onboard method to supply vacuum to the manifolds.

\subsection{Design Criteria Calculations}

Using the design criteria values outlined in the previous section, component sizing was determined to guide piece part and assembly level design. The calculations associated with the values in Table 2 are found in the appendices. These values will be used to finalize system designs and as a comparison metric in the vehicle testing described later in the report.

Table 2: Design criteria calculation results

\begin{tabular}{lll}
\hline Criteria & Value & Notes \\
\hline Vacuum Force Required [lbf] & 32.50 & Net force required to climb a vertical surface \\
Wall Area, Vacuum [in. ${ }^{2}$ ] & 2.50 & Total area of surface exposed to vacuum \\
Torque, Each Tread [in. ${ }^{*}$ lbf] & 15.3 & Net torque required to drive each tread \\
Torque, Each Motor [in. $\left.{ }^{*} \mathrm{lbf}\right]$ & 7.7 & Minimum torque required for each motor in a pair \\
\hline
\end{tabular}




\section{DETAIL DESIGN AND COMPONENT SELECTION}

The initial design criteria calculations provided valuable data to guide the design of the component parts of the vehicle. The following sections examine the design decisions that allowed for the form and function of the final prototype vehicle.

\subsection{Tread Design}

Essential for the operation of the prototype as well as the primary component of the unique vehicle locomotion/adhesion design, the treads are constructed from modified industrial timing belts. Simultaneously the most expensive single part on the vehicle and the component with the longest lead time, the high friction, perforated treads desired must be manufactured custom in a specialty belt shop. Due to these constraints, the treads were the first finalized design on the vehicle, and their specifications drove the design of the majority of other vehicle components.

\subsubsection{Vacuum Perforation Design}

Using the required exposed wall area of 2.50 in. $^{2}$ as a guide, several possible methods of perforating the drive treads were considered. The three most likely methods are shown below in Figure 15. Each of these methods have the same specific area (vacuum area per unit tread length) of 0.22 in. ${ }^{2} / \mathrm{in}$, requiring about 6 in. of tread length to be exposed to vacuum on each side of the vehicle. Each method's advantages and disadvantages are discussed briefly below. 


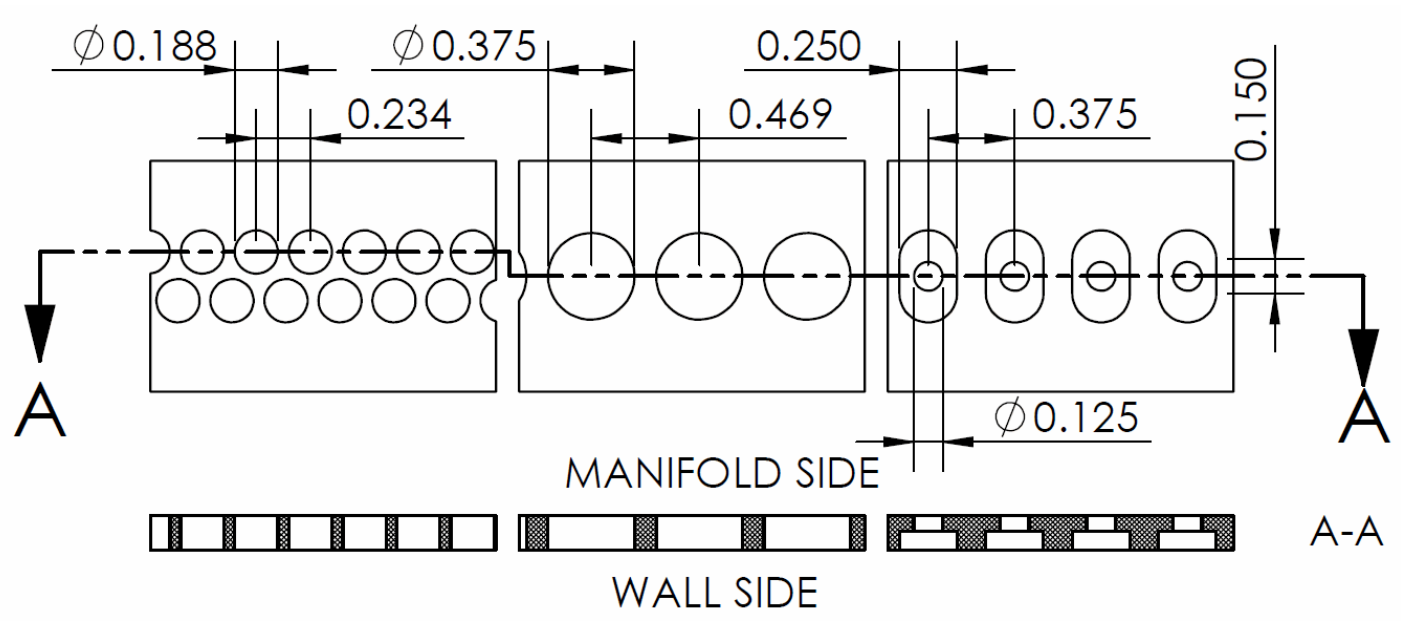

Figure 15: Three comparable tread perforation designs

Staggered Holes - The benefit that two or more rows of small, staggered holes provide is a high specific area while still allowing discrete vacuum sections to be closely spaced along the belt without the risk of a single perforation hole overlapping into two separate manifold sections, breaking manifold independence. Several disadvantages of this method include a large length of sealing edge required around a large number of hole features and a wide sealing width required between the manifold and the tread running surface.

Single Row of Holes - A single row of larger holes provides a similar manifold sealing width to the staggered hole design, but reduces the length of sealing edge between the wall and tread as well as the number of features. This would provide a cost reduction as well as improvement in design simplicity. However, in order to prevent breaching of two adjacent manifolds at a single time by one of the larger holes, the spacing of the manifolds would need to increase, lengthening the vehicle and adding weight.

Counter-bored Slots - Using a counter-bored slot in the belt backing material paired with a small through hole in the belt base material allows for a large vacuum surface area to be obtained while reducing the width of the sealing required between the manifold and the tread by $2 / 3$ when compared to the alternate designs. This allows for narrower and lighter treads, less critical seal area on the vehicle, and thus a greater possibility of successful adhesion. 
Additionally, as the through-holes in the base material are smaller, fewer of the fiberglass reinforcement strands must be severed during the perforation process, increasing the strength of the final belt when compared to larger hole patterns. While the added manufacturing complexity introduced by the additional machining operations is undesirable, the cascading weight and cost benefits of a narrower tread ultimately caused this design to be the best option for the prototype vehicle.

\subsubsection{Tread Base Material Selection}

A toothed belt, or timing belt, is the clear choice of belt type for power transmission in this application, due to the abundance of options commonly available in industry. Several companies nominally offer custom belt services that meet the requirements of the prototype. Each company offers a similar range of belt materials and constructions as outlined below. The possible options for belt base construction are outlined in qualitative terms in Table 3.

Table 3: Tread manufacturing options

\begin{tabular}{|c|c|c|c|c|}
\hline Type & Price & $\begin{array}{l}\text { Lead } \\
\text { Time }\end{array}$ & $\begin{array}{l}\text { Interrupts } \\
\text { Perforation? }\end{array}$ & Notes \\
\hline Pin Spliced & Low & Low & Yes & Belt ends spliced with metal pins \\
\hline $\begin{array}{l}\text { Urethane Thermally } \\
\text { Welded }\end{array}$ & High & Med & No & $\begin{array}{l}\text { Belt ends thermally welded, creates } \\
\text { seam, lower strength/stiffness }\end{array}$ \\
\hline $\begin{array}{l}\text { Urethane Molded } \\
\text { Endless }\end{array}$ & High & High & No & $\begin{array}{l}\text { Molded belt with endless steel or } \\
\text { Kevlar reinforcement }\end{array}$ \\
\hline $\begin{array}{l}\text { Neoprene Thermally } \\
\text { Welded }\end{array}$ & Med & Med & No & $\begin{array}{l}\text { Belt ends thermally welded, creates } \\
\text { seam, lower strength/stiffness }\end{array}$ \\
\hline $\begin{array}{l}\text { Neoprene Molded } \\
\text { Endless }\end{array}$ & Med & Med & No & $\begin{array}{l}\text { Molded belt with endless fiberglass } \\
\text { reinforcement }\end{array}$ \\
\hline
\end{tabular}

Based on research and discussions with the F.N. Sheppard representatives, the choice for this application is the Neoprene Molded Endless construction. Fiberglass-reinforced neoprene provides the strength and stiffness required to withstand the required tension on the belt, while allowing for a series of perforations unbroken by seams. The additional lead time (and higher cost) of the urethane option eliminated it from consideration. 


\subsubsection{Tread Backing Material Selection}

One of the most critical components of the vehicle, the backing material on the treads must be a high friction layer that provides sufficient grip against the climbing surface, while also being compliant enough to form and seal to small imperfections in the surface. In order to determine the proper material choice, several approaches were undertaken.

F.N. Sheppard supplied samples of their preferred high friction backing materials in order to facilitate testing. Using an electronic spring scale, a set of calibration weights, and a formed steel sled, all ten materials (and 14 material configurations in total) were tested for static friction coefficient against a representative slab of sheetrock. The full test report is included in the appendices.

To supplement the physical validation test performed, several resources provided tabulated friction data for their backing materials. Another timing belt manufacturer, Gates Mectrol, supplied this data in their design pamphlet seen in reference [17].

Table 4: Tread backing material options

\begin{tabular}{lllclcc}
\hline Material & $\begin{array}{l}\mu, \\
\text { Test }\end{array}$ & $\begin{array}{l}\mu, \\
\text { Lit. }\end{array}$ & Machinable? & $\begin{array}{l}\text { Hardness, } \\
\text { Shore A }\end{array}$ & $\begin{array}{l}\text { Vacuum } \\
\text { Suitable? }\end{array}$ & $\begin{array}{l}\text { Consultant } \\
\text { Recommended }\end{array}$ \\
\hline Black Rubber & 1.05 & -- & & $30-35$ & $\mathrm{X}$ & \\
Sponge Urethane & 0.94 & -- & $\mathrm{X}$ & Low & & \\
Natural Rubber & 0.93 & 1.5 & $\mathrm{X}$ & 40 & $\mathrm{X}$ & $\mathrm{X}$ \\
Linatex & 0.89 & 1.6 & $\mathrm{X}$ & 38 & $\mathrm{X}$ & $\mathrm{X}$ \\
Sponge Neoprene & 0.89 & 0.9 & $\mathrm{X}$ & $20-40$ & $\mathrm{X}$ & \\
\hline
\end{tabular}

Upon examination of Table 4, a summary of the five highest friction materials tested juxtaposed with several other factors and recommendations, Linatex was chosen as the backing material for the prototype treads. Linatex is a custom high performance rubber produced by The Weir Group. Specially formulated for high wear/high friction in conveying applications, it is used widely in industry and was recommended by F.N. Sheppard representatives. In my tests, its friction properties were virtually identical to the two sponge materials and the natural rubber. During review of industry literature, however, Gates Mectrol ranked the friction coefficient of 
Linatex higher than their natural rubber material. Machinability according to F.N Sheppard, hardness, and vacuum suitability (determined by open or closed cell structures in the material), provided the remaining data necessary make the decision to pursue Linatex as the designed tread backing.

\subsubsection{Final Tread Dimensions}

Table 5: Tread dimensions and specifications

\begin{tabular}{ll}
\hline Dimension & Value \\
\hline Material & Neoprene 240 Belt with 1/8 in. Linatex Backing \\
Weight, Each [lbf] & 0.22 \\
Perforation Pitch [in.] & 0.375 \\
Number of Perforations & 64 \\
Backing Material Thickness [in.] & 0.125 \\
Tread Pitch [in.] & $0.375(\mathrm{~L})$ \\
Pitch Length [in.] & 24 \\
Number of Teeth & 64 \\
Tread Width [in.] & 1.25 \\
Width of Teeth, Total [in.] & 0.750 \\
Width of Removed Teeth [in.] & 0.500 \\
\hline
\end{tabular}

The detail design of the treads to the specifications shown in Table 5 are shown in Figure 16. This task was completed quickly to allow for the lengthy lead time required for tread production.
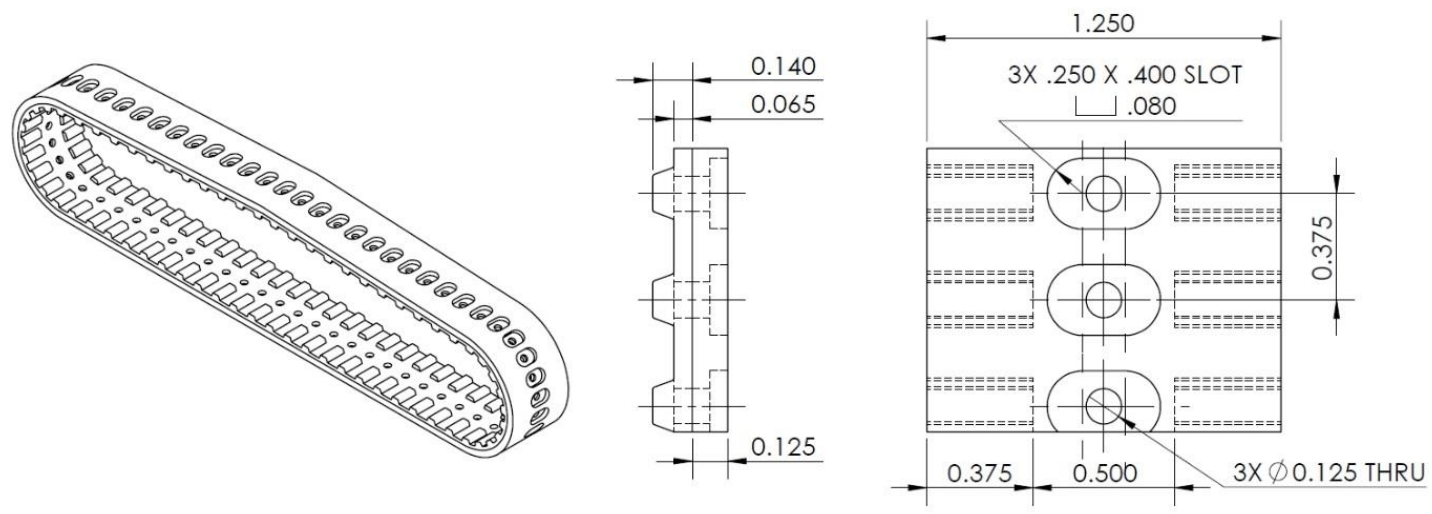

Figure 16: Final tread dimensions 
As discussed above, belt perforation details were derived in an iterative process using design criteria calculations, belt supplier info, and design capabilities for other parts in the vehicle. Perforation spacing of 0.375 in. was determined iteratively to allow sufficient vacuum area to be achieved in 6 in. of manifold length using 0.400 in. long slots with 0.25 in. radiused ends. Slot counter-bore depth of 0.080 in. was chosen to allow unrestricted airflow to all points of the counter-bore without risk that minor tread deformations could cause an unintended constriction in the counter-bored region. Backing material thickness of 0.125 in. was driven by counter-bore depth and validated using belt flexibility design tools provided by F.N. Sheppard seen in reference [21], using the chosen drive pulley diameter. The relatively thick backing should provide additional compliance to form to uneven surfaces.

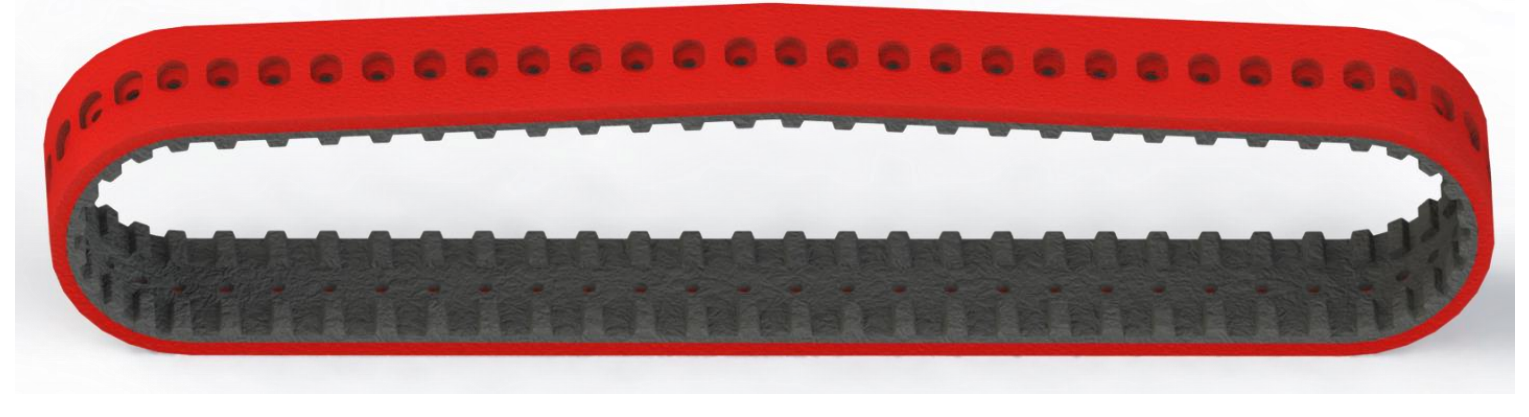

Figure 17: Render of the final tread design

Additional critical tread dimensions were determined. Tread pitch, or the spacing of the individual timing belt teeth, was chosen in an iterative process with the perforation design. A pitch designation " $L$ " designates a 0.375 in. spacing of the teeth, which aligns with the spacing of the belt perforation. This was chosen to ensure that any stiffness and flexibility variations between toothed and empty belt locations would be uniform across all perforation locations, giving a more predictable and uniform sealing surface to the vacuum manifold.

The tread pitch length was chosen to be the length sufficient to integrate a vacuum manifold with the required length of vacuum chamber between drive wheels, while allowing sufficient part clearances. 6 in. of vacuum chamber length were required to fulfill the design 
criteria, which, when part clearances and seal surface length were added, increased manifold part length to 7.15 in. Thus, setting center-to-center distance at 9 in. with the chosen 16 tooth drive pulleys resulted in a pitch length of 24 in. These values then directly determined the number of teeth (and holes) along the length of the belt. At 0.375 in. pitch and 24 in. pitch length, the belt will include 64 teeth and 64 perforations along its length.

Tread width was determined with input from sizing data available from Gates Mectrol, reference [22]. An allowance of $0.75 \mathrm{in}$. of total tread width, split into two $0.375 \mathrm{in}$. runs on either side, should permit an effective tension on the belt of approximately $100 \mathrm{lbf}$. This estimate, conservatively assuming a welded-end belt, provides a large safety factor of 6.5 over the estimated tread force of $15.3 \mathrm{lbf}$ calculated from vacuum-induced friction and vehicle weight. This buffer will allow for substantial tensioning or frictional exceedances without risk of slipping or damaging the belt teeth. A sliding toothless bed width of 0.500 in. will allow sufficient sealing area on either side of the 0.125 in. tread perforation holes to provide a high chance of achieving a good seal, driving the total belt width to 1.25 in.

\subsection{Vacuum Manifold Design}

Responsible for applying the vacuum pressure generated by the eight venturi pumps to the treads themselves, the vacuum manifolds are a critical part for vehicle functionality Several primary design aspects drove the development of the final manifolds.

Primarily, the manifold must provide enough exposed vacuum length to satisfy the design criteria. As discussed above, 6 in. of vacuum length is required, and that length will be split into four separate sections, each fed by an independent venturi pump. Some spacing between each manifold section is required to ensure no hole overlap between sections. 
Stiffness is critical to ensure that the manifold does not deflect due to the forces or pressures exerted on it during operation. Any deflection could cause the sealing surface to become deformed, interrupting the seal.

Friction between the manifold and the sliding tread bed should be minimized in order to reduce required motor torque, reducing the chance of tread buckling and other unwanted behaviors as it slides past the manifold.
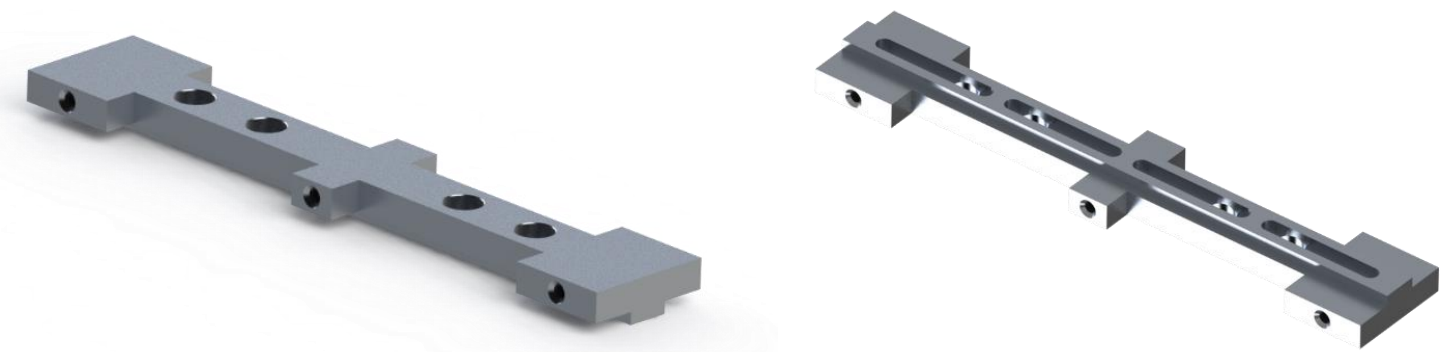

Figure 18: Two views of the vacuum manifold design

Considering each critical design component, the lightweight, four-section manifold displayed in Figure 18 was designed. Constructed of aluminum due to its stiffness and machinability characteristics, the manifold consists of an embossed central rail, sized to fit within the central channel of removed teeth on the tread, into which the four manifold slots are machined. Each independent slot is plumbed with a 1/8-27 NPT push-to-connect elbow fed by a $.25 \mathrm{in}$. OD vacuum line from the venturi pumps. In addition to providing the necessary seal to the treads, the sides of the running rail will interact with the belt teeth to ensure the belt remains centered during lateral force maneuvers, such as skid steering. In order to reduce friction between the manifold and the treads, adhesive-backed PTFE (Teflon) pieces will be applied to the main running surface as well as the sides of the rail. Critical specifications of the manifold are tabulated in Table 6. 
Table 6: Vacuum manifold specifications

\begin{tabular}{ll}
\hline Property & Value \\
\hline Material & $6061-$ T6 Extruded Bar \\
Weight [lbf] & 0.19 \\
Number of Slots & 4 \\
Length of Slots, Each [in] & 1.5 \\
Width of Slots [in] & 0.1875 \\
Distance Between Slots [in] & 0.1875 \\
Overall Length [in] & 7.15 \\
Pneumatic Fittings & 4x 1/8-27 NPT, Push-to-Connect Elbows \\
Friction Layer & PTFE Sheet, Adhesive Backed, .030 Thick \\
\hline
\end{tabular}

Efforts were made to reduce part size and weight. Non-essential metal is removed along both sides of the manifolds. Additionally, the 1/8-27 NPT holes shown in Figure 19 are designed to be close bottoming, reducing the required depth of hole by approximately a factor of 2 to achieve full thread engagement. Because of this, a two-stage tap process must be used to accommodate the push-to-connect fittings. Refer to the manufacturing sections for more information on this non-standard process.
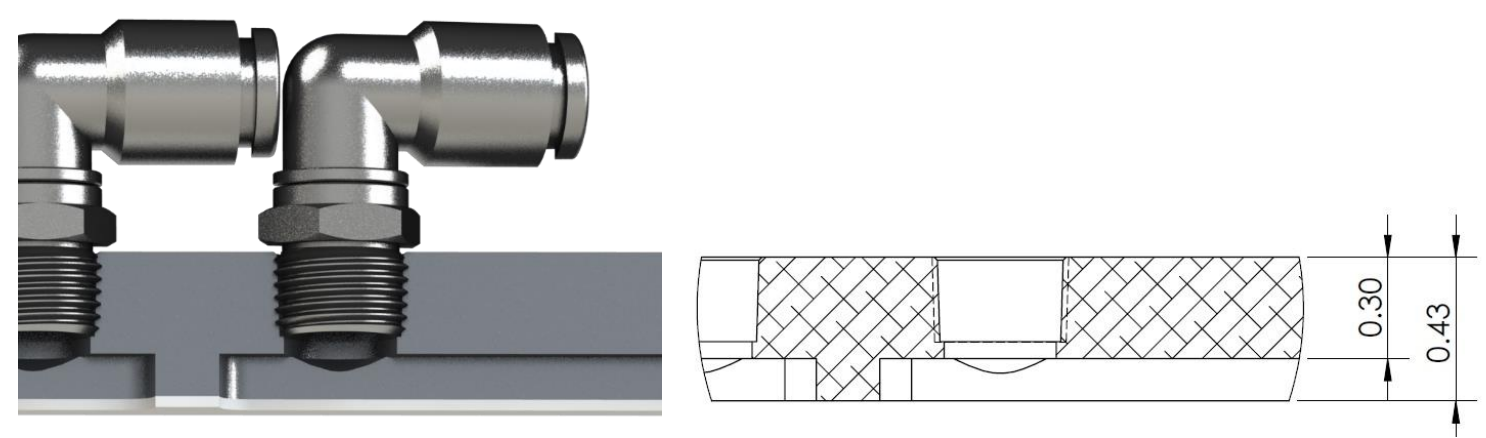

Figure 19: Detail views of the short, bottoming NPT tapped holes in the manifold 


\subsection{Venturi Choice}

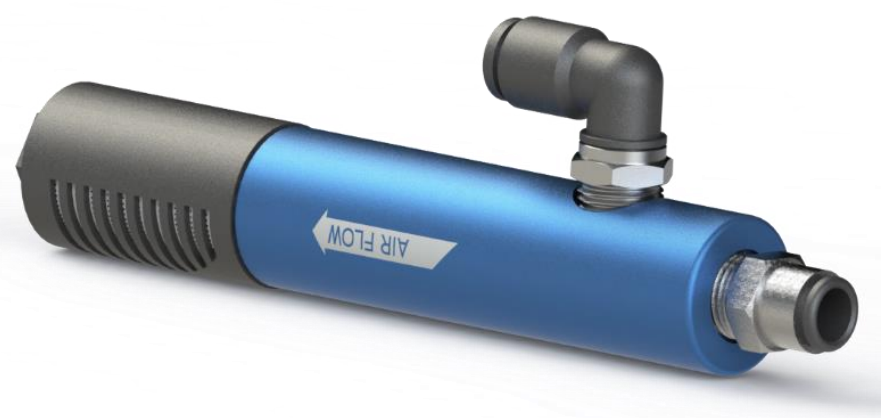

Figure 20: Render of the Vaccon JS-90M-AA4 venturi pump

The venturi system converts the input air supply pressure to the vacuum pressure required for the vehicle adhesion. The venturi system used on previous iterations [15], and thus the hardware available for use, are eight Vaccon JS-90M miniature venturi pumps. Rated to a high vacuum level of up to $28 \mathrm{inHg}$ and currently outfitted with the small, integrated -AA4 silencers and push-to-connect fittings for input and vacuum ports, the Vaccon venturis described in Table 7 meet the requirements of the project as-is and will be repurposed.

Table 7: Vaccon JS-90M-AA4 Specifications

\begin{tabular}{ll}
\hline Property & Value \\
\hline Venturi Type & Vaccon JS-90M-AA4 \\
Input Air Pressure [psi] & 80 \\
Maximum Vacuum [inHg (psi)] & $28(13.75)$ \\
Evacuation Rate [s/ft $\left.{ }^{3}\right]$ & 10.5 \\
Weight as Pictured [lbf] & 0.13 \\
Cost, Each (Excluding Fittings) & $\$ 71.50$ \\
\hline
\end{tabular}

\subsection{Pressure Manifold Choice}

The 80 psi input air for the venturi pumps must be supplied from one air source to all eight pumps simultaneously. Custom manifolds could be manufactured, but with limited machining time and facilities, as well as the added cost of purchasing at least nine separate pneumatic fittings, an off-the-shelf option is the superior choice. 


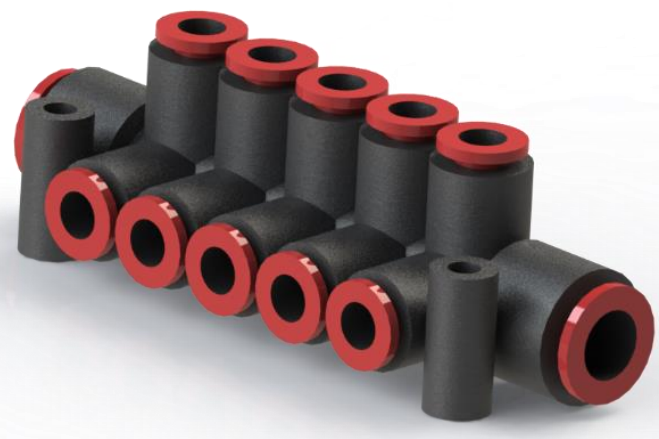

Figure 21: Render of the SMC Pneumatics KM11-07-11-10

Though not ideal, as the SMC pressure manifold will need the extraneous $0.375 \mathrm{in}$. fitting and two of the 0.25 in. fittings to be plugged, the light weight and low cost of the manifold made it an easy choice for the vehicle. Table 8 provides an overview of the manifold's properties.

Table 8: Pressure manifold specifications

\begin{tabular}{ll}
\hline Property & Value \\
\hline Part & SMC Pneumatics KM11-07-11-10 \\
Weight [lbf] & 0.08 \\
Pressure Ports & $2 \times 0.375$ OD Tubing, Push-To-Connect \\
Exhaust Ports & $10 x 0.25$ OD Tubing, Push-To-Connect \\
Proof Pressure [psi] & 435 \\
Price & $\$ 23.37$ \\
\hline
\end{tabular}

\subsection{Motor Choice}

Considerations when choosing locomotion hardware for the vehicle consisted of motor size, overall weight, and torque capability. Two motors will be used on each tread unit. This ensures that the tread is always being pulled past the vacuum manifold, as pushing could cause local buckling of the tread as the frictional force increases between the manifold and belt backing, potentially breaking the vacuum seal.

To ease integration, the chosen motor should be able to fit within the projected outline of the primary drive pulleys. Additionally, it should be face-mountable and should be able to direct drive the primary pulleys in order to minimize additional brackets, couplers, and axles. The 
outlined design criteria specify a required torque per tread of 15.3 in.*lbf, or approximately 7.7 in.*lbf per motor.

As the vehicle is able to be powered, at least initially, by a remote power supply, voltage and current requirements are flexible. Note that while vehicle speed is not a driving concern for this proof-of-concept, it is important to determine the range of speeds that will be available.

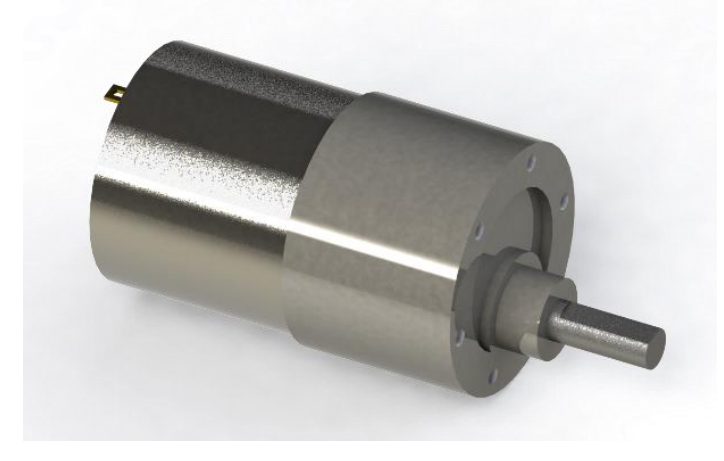

Figure 22: Render of the Pololu 131:1 37D Metal Gearmotor

The chosen motor, rendered in Figure 22, is a high-reduction brushed DC gearmotor supplied by the robotics vendor Pololu. Pololu's motor met or exceeded all requirements outlined by the design criteria. Low amperage $12 \mathrm{VDC}$ power requirements facilitate easy power and control. Rated at 80 RPM, the maximum vehicle speed (assuming a 1 -in. drive radius) is approximately $8.35 \mathrm{in} . /$ second. An eccentrically aligned output shaft allows the motors to be positioned such that their centers of gravity are below the midplane of the drive pulleys, which helps keep the center of gravity of the vehicle as close to the climbing surface as possible, reducing peel-off forces. Most importantly, the Pololu motor provides 15.6 in.*lbf of torque in a compact, lightweight $0.45 \mathrm{lbf}$ package. With two motors on each tread, an additional safety factor on torque requirements of just over 2 will allow for any unanticipated friction and ensure the vehicle does not stall. 
Table 9: Pololu Gearmotor Specifications

\begin{tabular}{ll}
\hline Property & Value \\
\hline Motor Name & Pololu 37D 131:1 Metal Gearmotor \\
Dimensions [in. x in.] & $1.45 \mathrm{D} \times$ x 2.25L \\
Weight [lbf] & 0.45 \\
Operating Voltage [VDC] & $6-12$ \\
Stall Torque [in.*lbf] & 15.6 \\
Stall Current [A] & 5 \\
Free Run Speed [RPM] & 80 \\
Free Run Current [A] & 0.3 \\
Mounting Screws & $6 \times \mathrm{M} 3$ \\
Shaft & $6 \mathrm{~mm} \mathrm{D-Shaft}$ \\
Cost Each & $\$ 24.95$ \\
\hline
\end{tabular}

\subsection{Treadpod Frame Design}

Design requirements for the treadpod frame include providing integration for two motors, two drive pulleys, one idler pulley and accompanying tensioner system, mounting and adjustment for the vacuum manifold, connection points to the central frame and opposite tread pod, and the required brackets and stiffeners to ensure adequate structural capability to withstand the vehicle's loading.

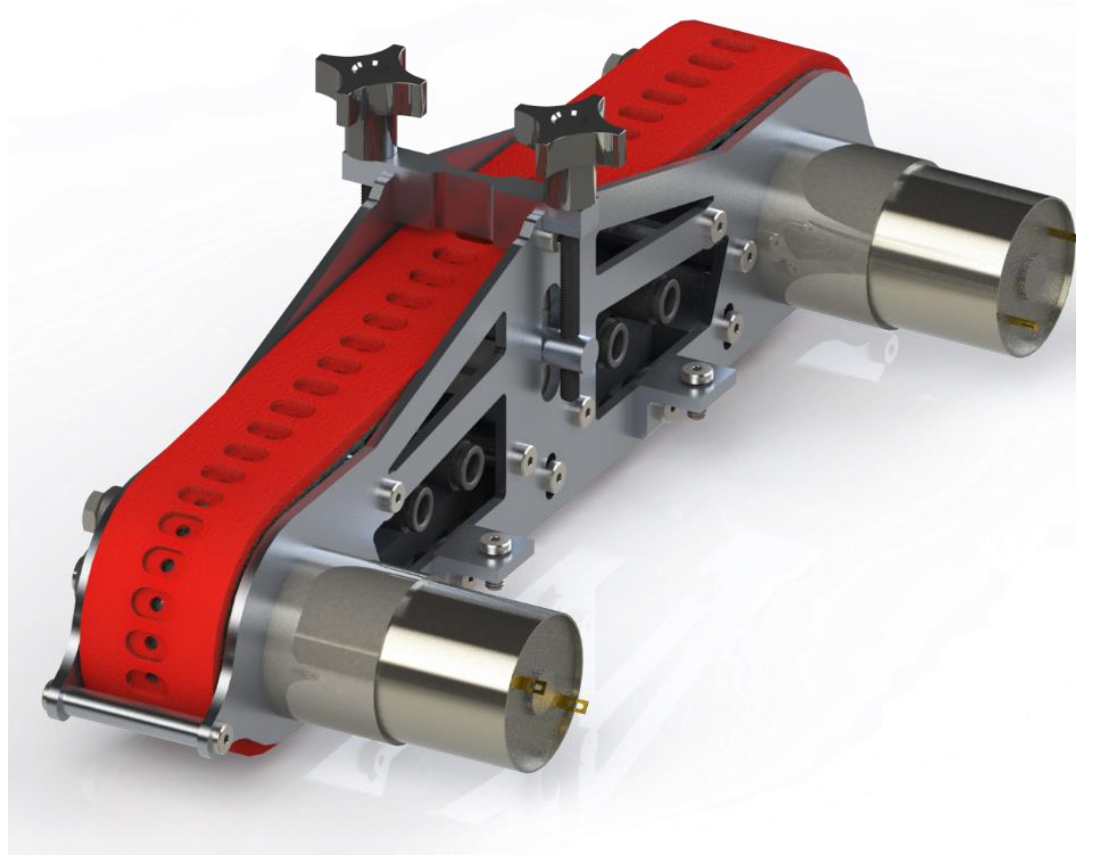

Figure 23: Render of a single treadpod 
From the beginning, a largely symmetrical, self-contained treadpod, as modeled in Figure 23 , was seen as the best way to simplify and reduce weight while ensuring that the vehicle was not performance-biased in any particular direction. Thus, two configurations of a similar part profile were designed; each treadpod would consist of the tread itself flanked by two frame pieces. Both configurations, shown in Figure 24, share fastener holes for the spacers, brackets, and stiffeners required for treadpod structure. Specifically, the shared features include accommodations for four structural standoffs, two shear support brackets, mounting holes and retaining features for the idler support bracket as well as the guide slot for the idler axle, and three slots to allow for vertical adjustment of the vacuum manifold relative to the tread.
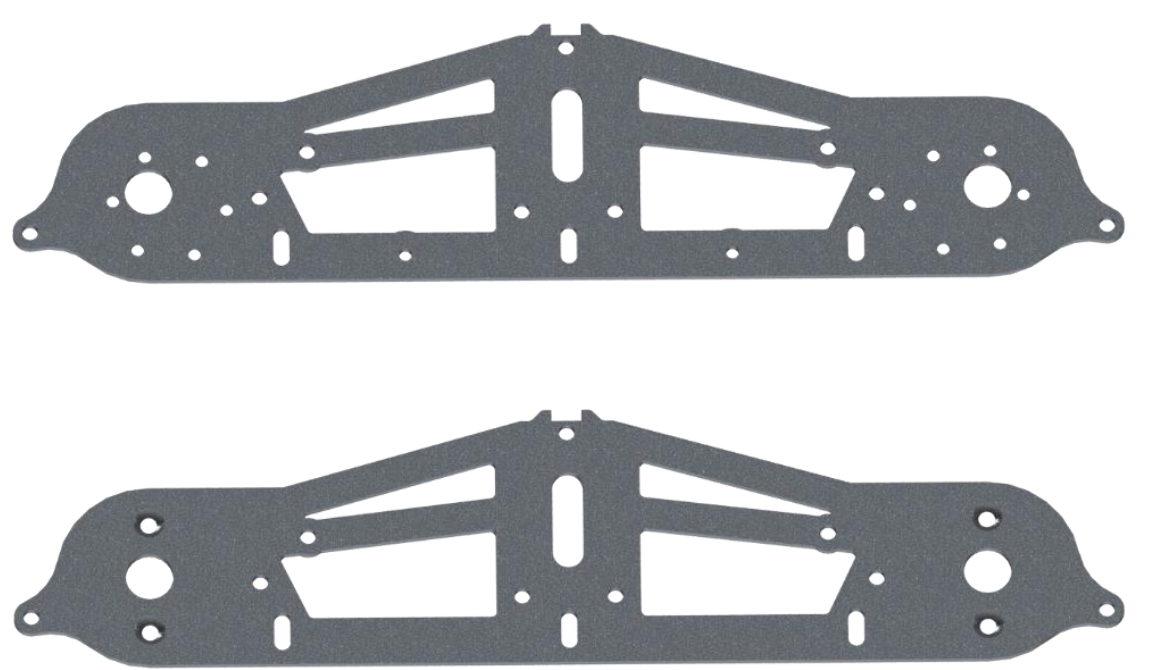

Figure 24: Renders of both frame configurations, motor-side (top) and outboard (bottom)

The first of the configurations would accommodate mounting features for the two Pololu motors and the connection to the remainder of the vehicle. The opposite configuration would provide mounting for the support bearing on the outboard drive pulley axle. As both the motors and the support bearings are mounted directly to the frame, the mounting hardware required countersinks as to not interfere with the rotation of the drive pulleys or the movement of the tread. 
As such, the parts are 0.100 in. thick to allow for adequate countersink depth without adding unnecessary weight.

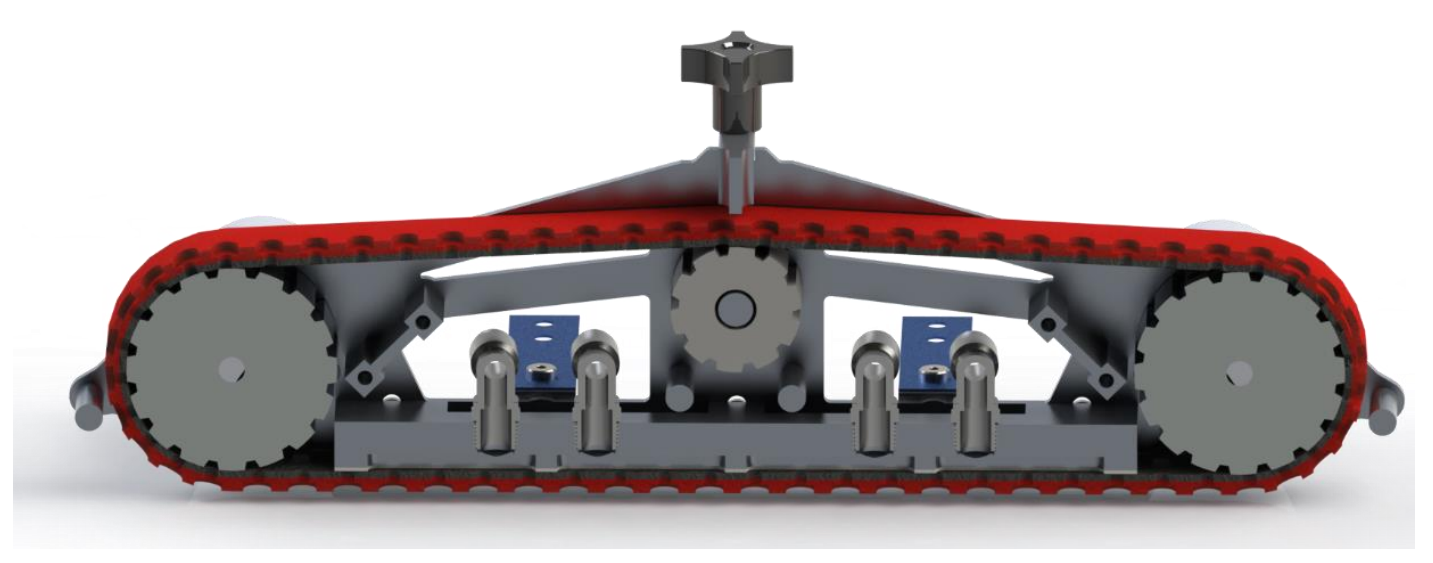

Figure 25: Cutaway render of a treadpod, showing internal structure

Structurally, the forces borne by the frame pieces include vehicle weight (15 lbf design), belt tension, and various frictional forces caused by the belt-to-manifold interactions. These stresses are insignificant compared to the yield strength of the aluminum. Buckling, however, is important to consider, as the belt tension could cause a .100 in. thick span to fail in buckling. Shortening unsupported spans through the addition of standoffs and stiffening the assembly with the shear supports ensures that the frame does not deflect out-of-plane. These supports can be clearly seen in Figure 25 and Figure 26. 

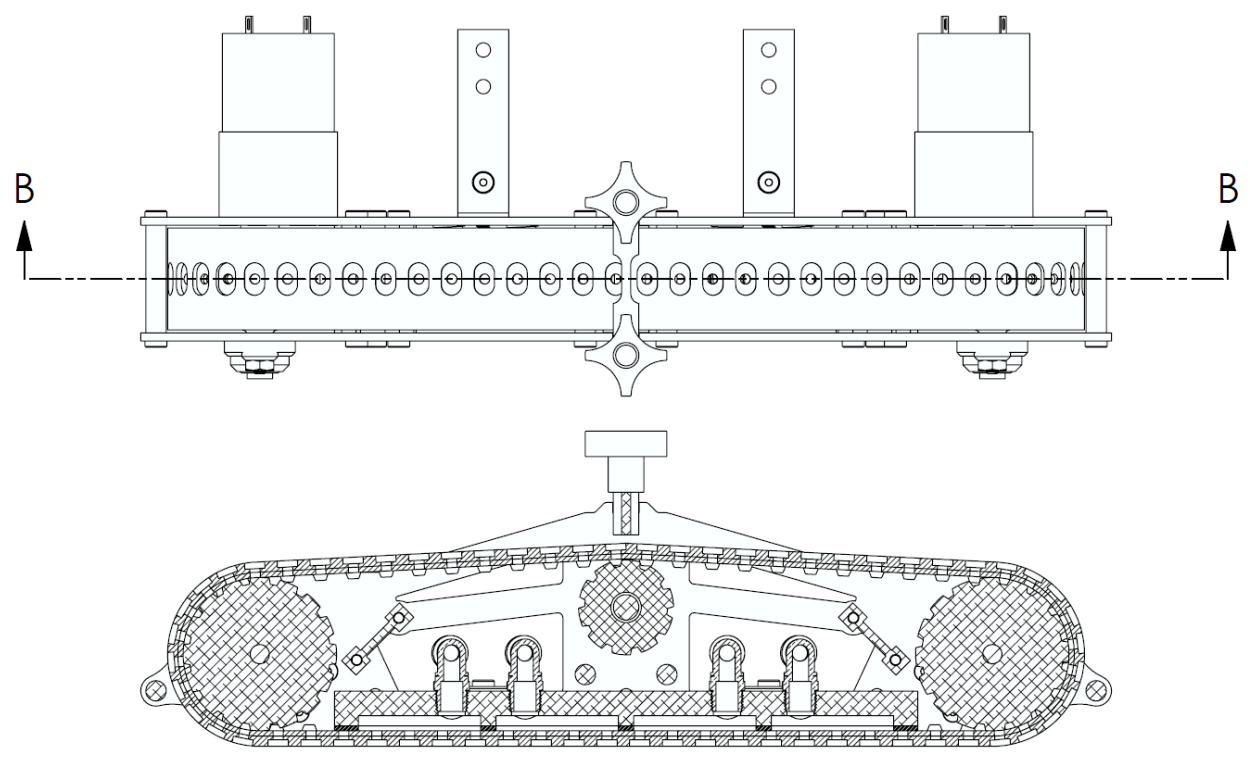

SECTION B-B

Figure 26: Cross section diagram of treadpod

Table 10: Treadpod frame specifications

\begin{tabular}{ll}
\hline Property & Value \\
\hline Material & $6061-$ T6 Sheet \\
Thickness [in.] & 0.100 \\
Weight [lbf] & 0.18 (Both Configurations) \\
Overall Dimensions [in.] & $11.75 \times 2.9$ \\
Motor Center-to-Center Distance [in.] & 9 \\
\hline
\end{tabular}

\subsection{Drive Pulley Design}

Sizing and design of the drive wheels combined inputs from the design criteria with integration and packaging concerns, weight, and material limitations provided by the belt manufacturer, F.N. Sheppard. Preliminary calculations were performed with a approximated drive wheel and tread radius of $1 \mathrm{in}$. A pulley with a diamter of slightly less than $2 \mathrm{in.}$ would, as determined during initial structural layout, provide sufficient area within the path of the treads to accommodate the necessary pneumatic fittings, idler assembly, and any other structure that must be contained within the treadpod itself.

Beyond integration concerns, F.N. Sheppard provides guidelines for minimum pulley diameter in Figure 27 when given belt backing material type and thickness. Linatex, which F.N. 
Sheppard considers a "Soft Type A" material, at 0.125 in. thickness requires a minimum pulley diameter of approximately 0.75 in., as conservatively extrapolated from the avialble information. Thus, a 2 in. diameter pulley will cause no problems with the belt or backing material.

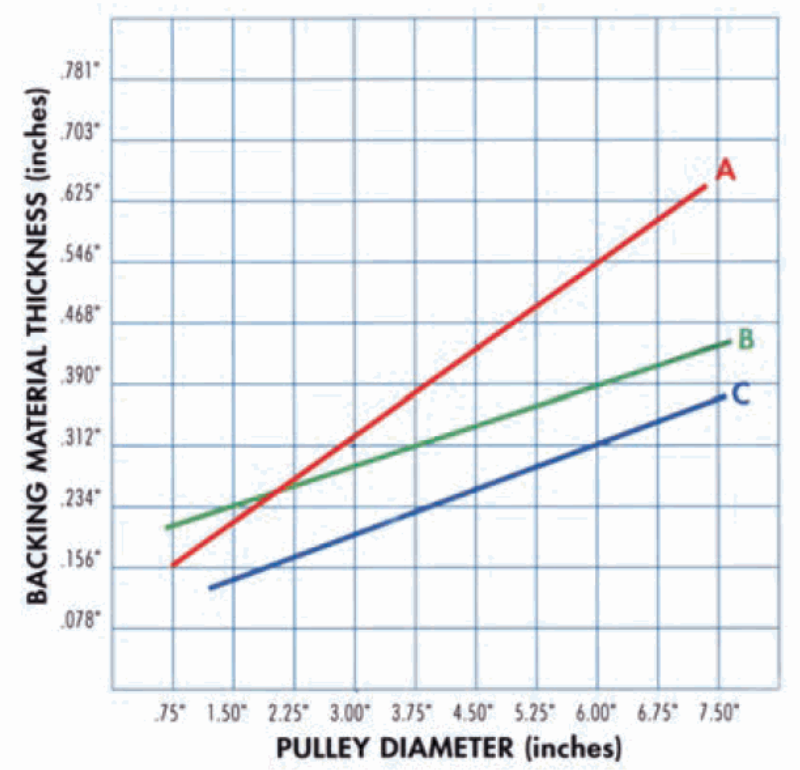

Figure 27: Chart of minimum pulley diameters provided by F.N. Sheppard [21]

The drive pulleys were sized to L-pitch, 16-tooth pulleys. This provided a pulley OD of 1.91 in., which, when accouting for the thickness of the belts, places the final drive diameter at just over 2 in.

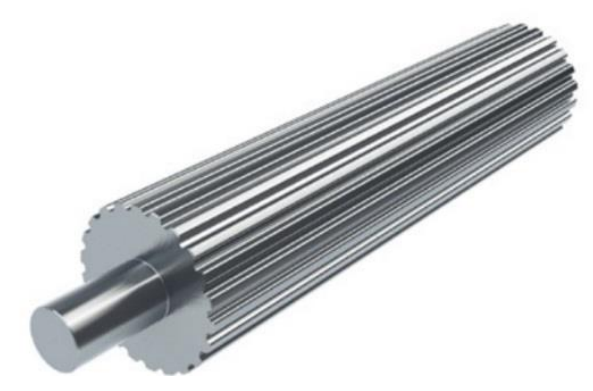

Figure 28: Timing belt pulley stock

Due to the unusual 1.25 in. width of the drive belts, falling between standard belt sizes of 1.0 in. and 1.5 in., off-the-shelf options for drive pulleys are rare and expensive to obtain. 
Additionally, the majority of timing belt pulleys are design for ground operations, where weight and size are not a concern. As such, many pulleys on the market are steel, and most include bulky set screw hubs and thick belt alignment flanges. As none of these aspects are desirable for this application, another option needed to be found.

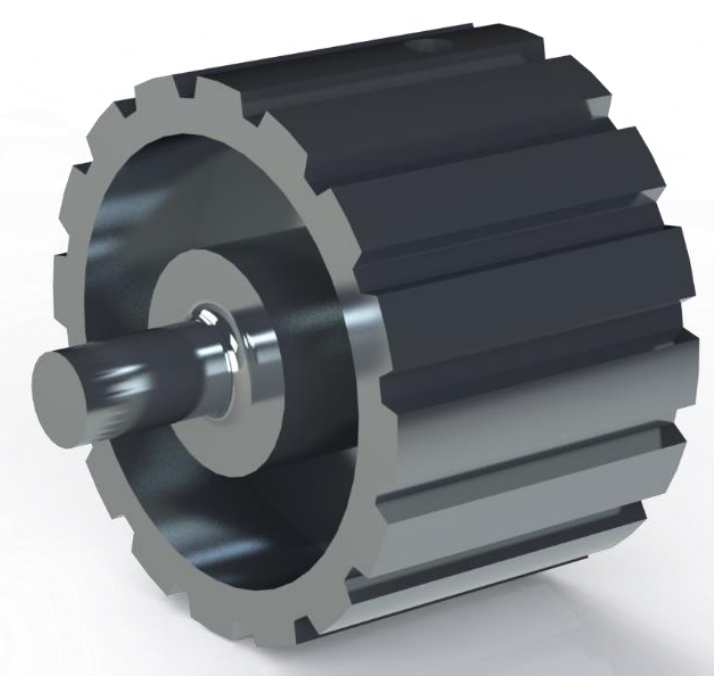

Figure 29: Render of the drive pulley

In order to create a custom pulley while avoiding the difficulty of machining the pulley teeth, the drive pulleys will be machined from aluminum timing belt stock, which is a type of offthe-shelf round stock with timing belt tooth profiles pre-machined into the outer surface. This stock can be purchased in the form shown in Figure 28. Postmachining will be performed on manual machines at Cal Poly in order to manufacture the correct width pulley and form the reamed hole for the motor axle as well as the stub shaft for the support bearing on the opposite side. In this way, no additional axles or parts are necessary to transmit torque from the motor directly through to the treads. A single \#8-32 set screw will be installed for a positive hold on the motor's $6 \mathrm{~mm}$ D-shaft, with tool access through a hole drilled in the top land of one of the pulley teeth. This assembly is illustrated in Figure 30. 


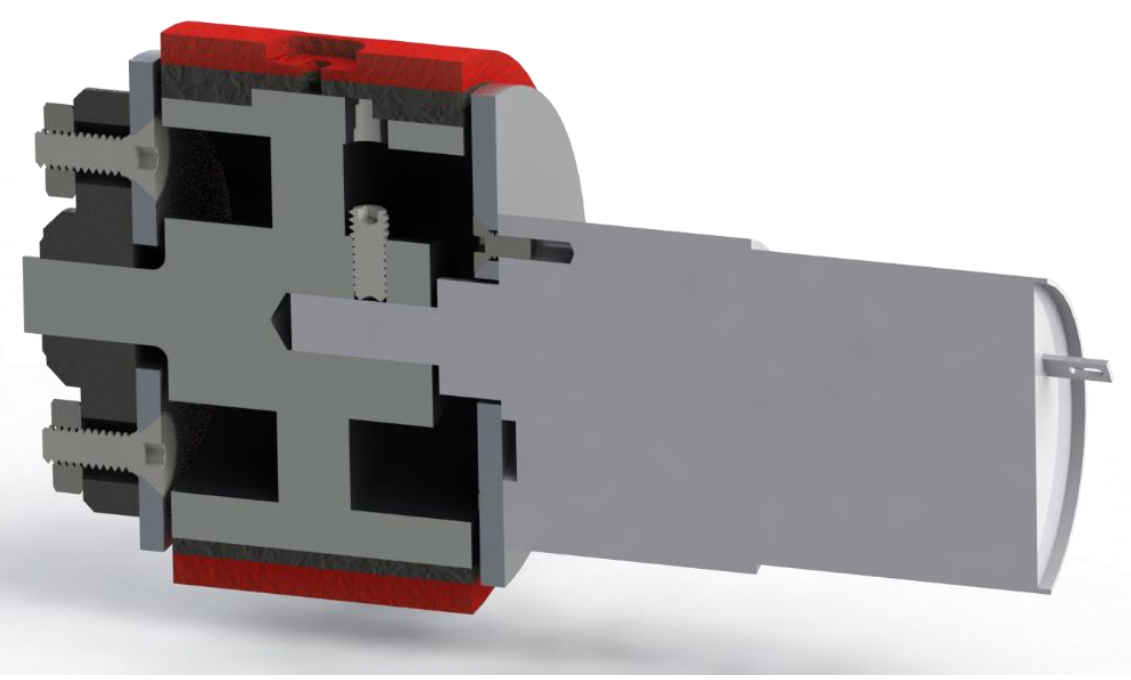

Figure 30: Section view of the drive assembly

Further weight reduction is accomplished by removing a large amount of extra radial material from both faces of the pulley. The central "spoke" of the wheel that remains to connect the toothed rim to the central hub is 0.30 in. thick, easily sufficient for torque transmission and stiffness requirements.

Table 11: Drive pulley specifications

\begin{tabular}{ll}
\hline Property & Value \\
\hline Material & Aluminum \\
Number of Teeth & 16 \\
Pulley Outer Diameter [in.] & 1.88 \\
Pitch Diameter [in.] & 1.91 \\
Weight, Each [lbf] & 0.19 \\
Weight Savings Over Solid Pulley, Each [lbf] & 0.13 \\
Face Width [in.] & 1.25 \\
Motor Shaft Hole Diameter [mm] & 6 \\
Support Axle Diameter [in.] & 0.3125 \\
\hline
\end{tabular}

\subsection{Support Bearing Choice}

In order to reduce the moment on the motor shaft, increase stiffness of the drive wheel, and prevent deflection, a bearing was chosen to support the outboard axle stub of the drive wheel. A flush-mounted pillow block style bearing would be the simplest to integrate and mount with the 
treadpod assembly. Two off-the-shelf options were found: a ball bearing style assembly in a cast zinc flange and a polymer spherical bushing in a polymer flange, both of which are shown in Figure 31. Rolling element bearings would be recommended for higher speed or higher load applications, but the light weight and smaller size of the polymer unit, in combination with the vehicle's limited loading and speed, made the polymer bushing a clear choice.
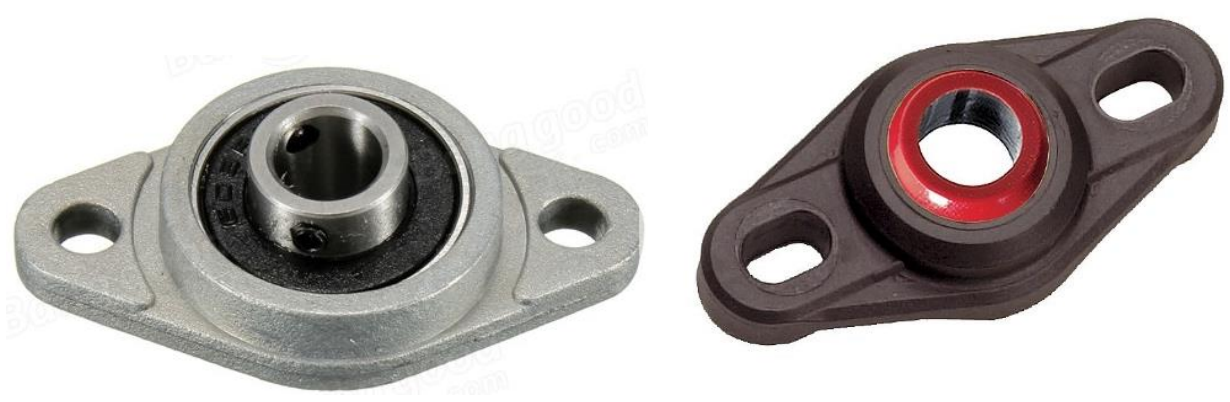

Figure 31: Images of the two support bearings considered

Table 12: Support bearing specifications

\begin{tabular}{lll}
\hline Property & Metal Ball Bearing & Polymer Bushing \\
\hline Manufacturer & China Generic & IGUS \\
Weight, Each [lbf] & 0.06 & 0.01 \\
Footprint [in. x in.] & $1.87 \times 1.02$ & $1.74 \times .82$ \\
Bore Diameter & $8 \mathrm{~mm}(0.315 \mathrm{in})$. & $0.3125 \mathrm{in}$. \\
Mounting Hardware & M6 & $\# 8-32$ \\
Cost, Each & $\$ 1.82$ & $\$ 3.13$ \\
\hline
\end{tabular}

\subsection{Tensioner Pulley Design}

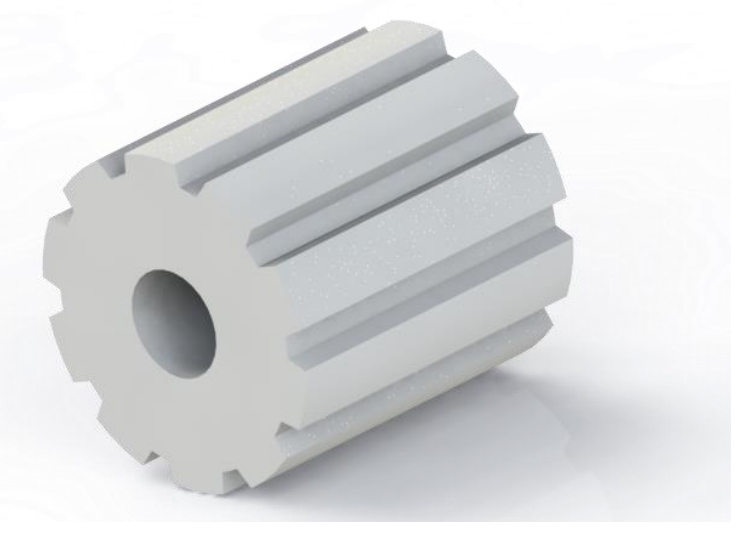

Figure 32: Render of the tensioner pulley 
The tensioner assembly includes a single idling pulley which can be adjusted to increase or decrease belt tension. Design concerns for the tensioner pulley are significantly simpler than the drive pulley, as no torque is being transmitted through the pulley itself. Weight and size are the main criteria for these parts. Thus, the smallest available pulley was chosen for the idler. Similar to the drive pulleys, the unusual 1.25 in. width of the belt showed the benefit of a partially custom pulley machined from 10-tooth pulley stock. This small size eases integration and minimizes weight, and still falls above the minimum pulley radius of $0.75 \mathrm{in}$. Unlike the drive pulleys, however, there are no tapped holes or set screws to prompt concerns of thread strength or durability. Thus, acetal or Delrin pulley stock is acceptable and saves approximately $48 \%$ in weight over a similarly sized aluminum pulley. Machining on the tensioner pulley is limited to facing to width on the lathe and drilling a though hole to accept the idler axle and the associated flanged bushings.

Table 13: Tensioner pulley specifications

\begin{tabular}{ll}
\hline Property & Value \\
\hline Material & Delrin \\
Number of Teeth & 10 \\
Pulley Outer Diameter [in.] & 1.164 \\
Pitch Diameter [in.] & 1.194 \\
Face Width [in.] & 1.25 \\
Weight, Each [lbf] & 0.06 \\
Though Hole Diameter [in.] & 0.375 \\
\hline
\end{tabular}




\subsection{Tensioner Assembly Design}

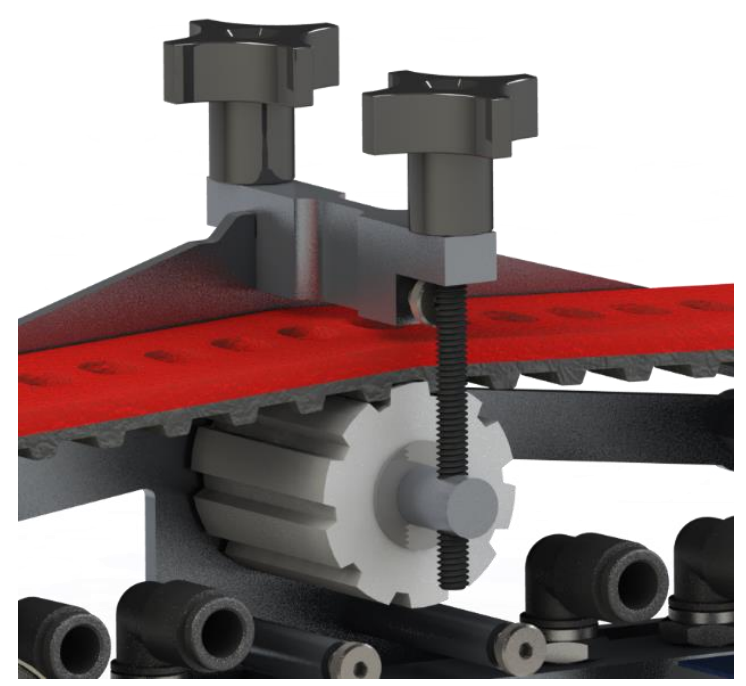

Figure 33: Render of the tensioner assembly

The role of the tensioner assembly is to adjust the position of the tensioner pulley in a controllable manner so that the drive belt can reach the desired tension. The obvious method to accomplish this is to raise the tensioner pulley's axis vertically into the underside of the belt. In order to avoid the asymmetric and mass-inefficient method of cantilevering the axle from one end, two \#8-32 threaded rods will be used on each assembly, with one on each side of the pulley. Thumb screw lobes on the top of the screws will allow for easy, tool-free adjustment. Tapped holes in the pulley's 0.3125 in. diameter axle will allow the thumb screws to, when adjusted simultaneously, raise or lower the pulley. A simple aluminum bracket above the belt will provide the support for the thumb screws, while also serving as an additional spacer for the treadpod frame pieces. To reduce frictional forces during rotation of the tensioner pulley about the axis, polymer flange bushings will be pressed into the ends of the pulley. The $0.030 \mathrm{in}$. width of the pulley flanges will also serve as spacers and axial bearing surfaces between the rotating pulley and the stationary treadpod frame pieces. All of these components are clearly visible in Figure 33. 
Table 14: Tensioner assembly specifications

\begin{tabular}{ll}
\hline Property & Value \\
\hline Vertical Adjustment Length, Measured from Tread Engagement [in.] & 0.53 \\
Effective Belt Pitch Length Adjustment [in.] & 0.10 \\
\hline
\end{tabular}

Due to the frame pod being designed nominally at tread pitch length, a relatively short tensioner travel would provide the additional tension for the vehicle. F.N. Sheppard's consultant was confident that $0.10 \mathrm{in}$. of additional pitch length would provide sufficient tension for the application. This short travel allowed the treadpod frame to remain small and light.

\subsection{Suspension Design}

Any torsional misalignment between two rigidly connected treadpods would inevitably raise one tread's sealing surface off of the wall, resulting in a broken seal. Due to the inevitability of manufacturing error at some level, a suspension system is recommended to allow both treadpods to be flush and flat against the climbing surface at all times. This suspension system should be designed to allow movement of one treadpod relative to the other in certain directions, while keeping critical alignment fixed, such as the parallelism of the treads. Figure 34 and Table 15 below capture the desired suspension characteristics.

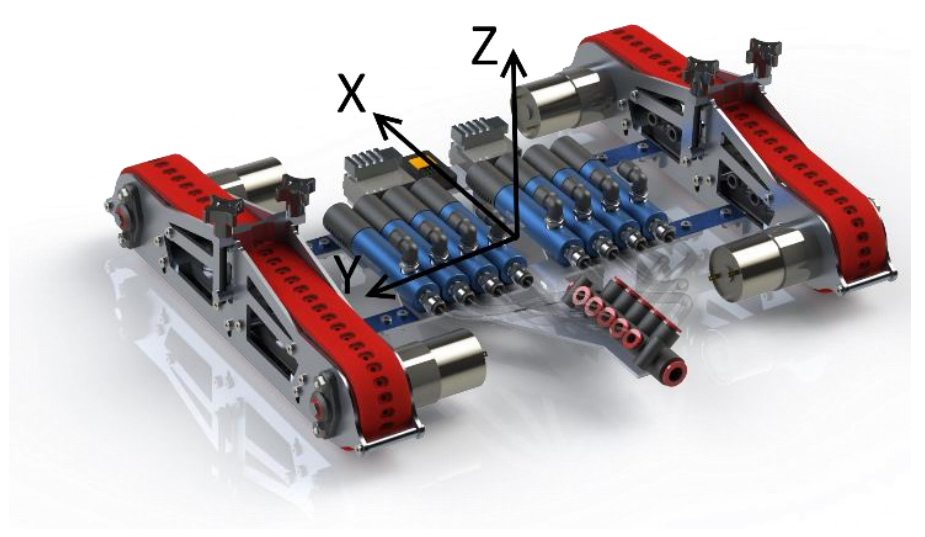

Figure 34: Full assembly render with overlaid coordinate axes 
Table 15: Desired suspension degrees of freedom

\begin{tabular}{lll}
\hline $\begin{array}{l}\text { Degrees of Freedom, Treadpod } \\
\text { Relative }\end{array}$ & Ideal Suspension States & Spring Flexure States \\
\hline $\mathrm{X}$ & Fixed & Fixed \\
$\mathrm{Y}$ & Fixed & Fixed \\
$\mathrm{Z}$ & Suspended & Suspended \\
Rotation about X & Fixed & Suspended \\
Rotation about Y & Suspended & Suspended \\
Rotation about Z & Fixed & Fixed \\
\hline
\end{tabular}

Many suspension designs were considered, including multilink systems with hobby grade shock absorbers and springs, flexible rubber bushings, coil springs, and two-axis hinges with end stops and bumpers. Following the design principles of simplicity, light weight, and manufacturability, the suspension method used for the vehicle consists of spring steel flexures. Spring flexure suspension will, unfortunately, allow for treadpod rotation along the vehicle's $\mathrm{x}$ axis as the flexures deflect. However, a key advantage of the spring flexure design is the ease of adjustment. A simple length or thickness change on the flexures can allow for manipulation of system stiffness, if required. The decision to pursue spring flexure suspension was based on the assumption that the suspension could be adjusted to fit the stiffness required for prototype operation.

Analysis was performed for flexure systems including a single, wide flexure plate at the center of each tread pod as well as a double-flexure system in order to determine an initial flexure size. Stiffness in both cantilever bending and flat plate torsion were compared and iterated over various flexure thicknesses, widths, and overhang lengths. The objective was to obtain stiffness on the same order of magnitude for each potential suspension compliance direction, so that the treadpods are able to adjust to any minor surface irregularities. Systems with two flexures provide much more control of fixed and suspended degrees of freedom, as well as being easier to integrate. Additionally, calculations show that a comparable stiffness single flexure design could be $50 \%$ or more heavier than the related double-flexure system. 

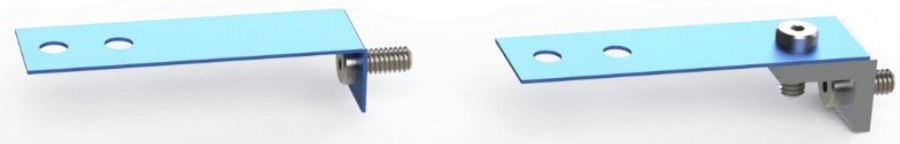

Figure 35: The preliminary (left) and final (right) suspension flexure designs

The preliminary design was a single, bent spring flange, as seen in Figure 35. In order to avoid the difficulty of tempering raw, annealed spring steel after forming, an additional aluminum bracket was designed to allow for the purchase of pre-tempered spring steel. The small weight increase was deemed worthwhile. The flexure design specifications are tabulated in Table 16.

Table 16: Final suspension specifications

\begin{tabular}{ll}
\hline Property & Value \\
\hline Material & 1095 Spring Steel, Blued and Hardened \\
Width [in.] & 0.625 \\
Length, Total [in.] & 2.25 \\
Length, Flexure [in.] & 1.18 \\
Spacing [in.] & 3.50 \\
Approx. Force to Deflect Treadpod .125 [lbf] & 11.1 \\
Approx. Torque to Twist Treadpod 3 ${ }^{\circ}\left[\mathrm{in} .{ }^{*} \mathrm{lbf}\right]$ & 13.5 \\
Weight, Full Suspension System [lbf] & 0.11 \\
\hline
\end{tabular}

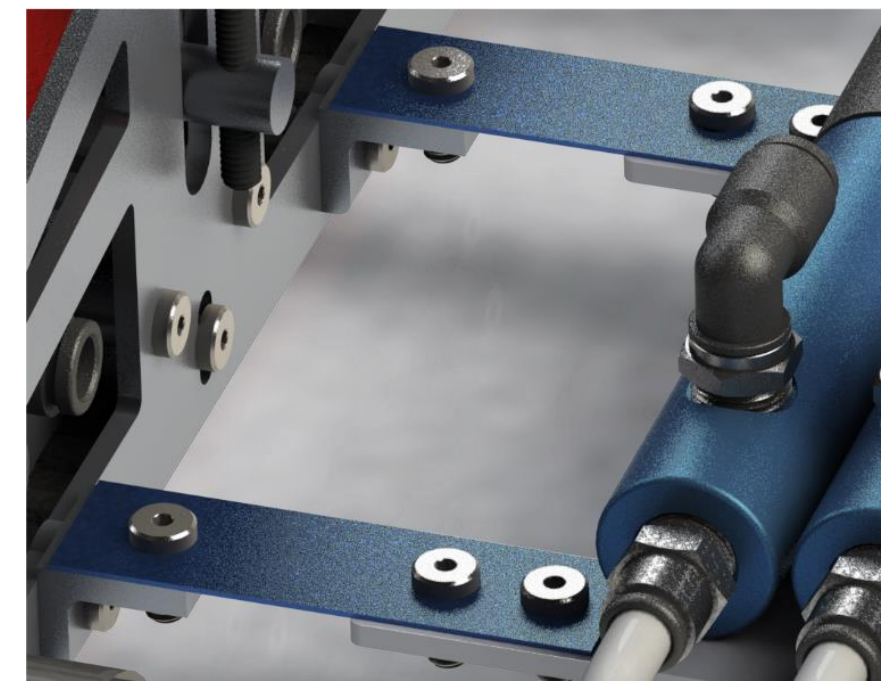

Figure 36: Render of the assembled suspension system 


\subsection{Central Frame Design}

Due to the self-contained design of the treadpods, the central frame of the vehicle simply must accommodate mounting for the pneumatic systems, including the pressure manifold and eight Vaccon venturis, as well as any control systems for the motors. Forces taken by the frame include the weight of the attached parts and minor forces transmitted by the suspension from the treadpods, including skid steering inputs. Thus, the frame was designed from the same 0.100 in. thick aluminum sheet as the treadpod frame pieces. Critical dimensions can be reduced to the mounting points for the suspension flexures, while secondary dimensions were determined by the physical size of the venturi pumps and electronics, seen in Figure 37.
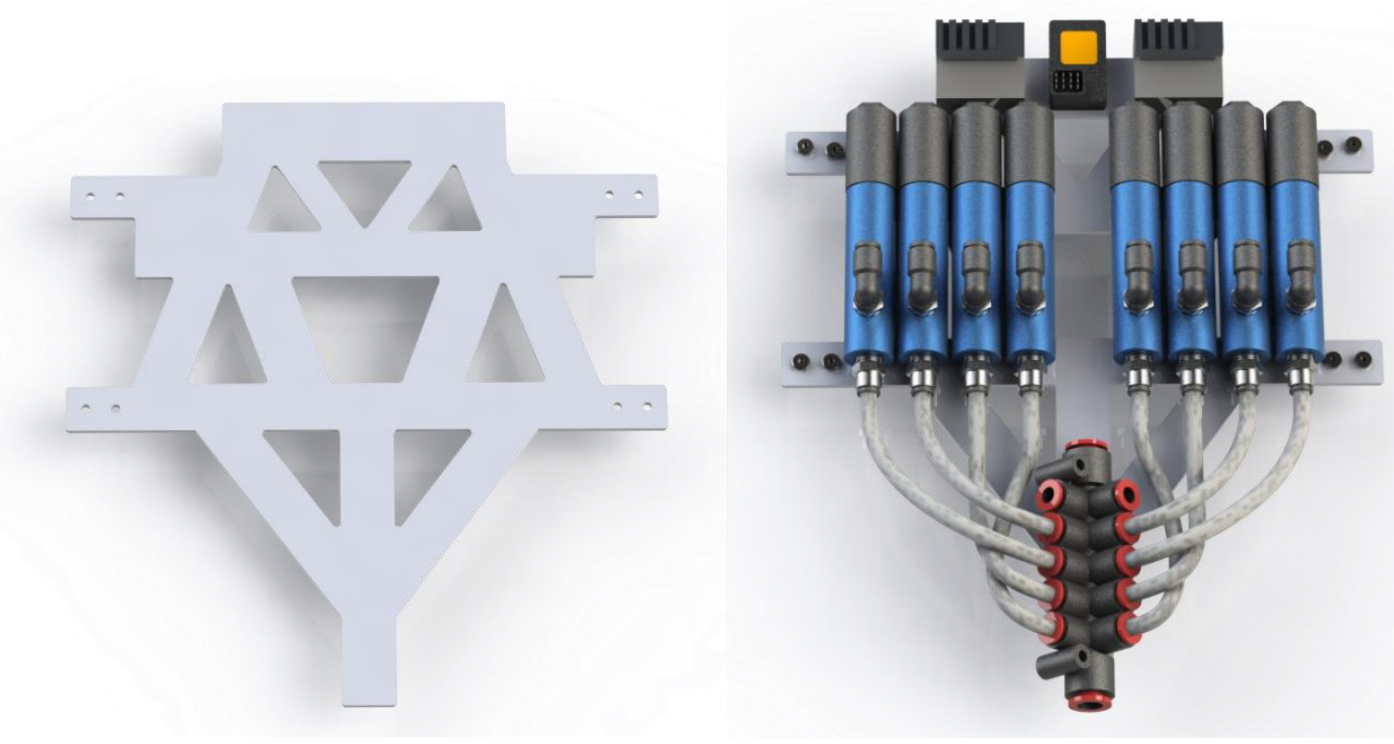

Figure 37: The central frame, bare (left) and assembled (right)

In order to reduce weight, reduce part count, and simplify manufacturing and assembly, no fasteners are required to attach any components on the frame. Instead, adhesive-backed cable tie mounts will be affixed directly to the frame itself, and each component is simply attached to the mounts with cable ties, allowing a tool-free assembly and easy maintenance if required. The three continuous horizontal rails on the frame render shown in Figure 37 will hold the cable tie 
mounts for the venturis and electronics, while the pressure manifold will be affixed to the vertical rail at the bottom of the image.

Table 17: Central frame specifications

\begin{tabular}{ll}
\hline Property & Value \\
\hline Material & $6061-$ T6 Aluminum Sheet \\
Thickness [in.] & 0.100 \\
Net Dimensions [in. $x$ in.] & $9 \times 9.75$ \\
Weight [lbf] & 0.13 \\
\hline
\end{tabular}

\subsection{Control System Choice}
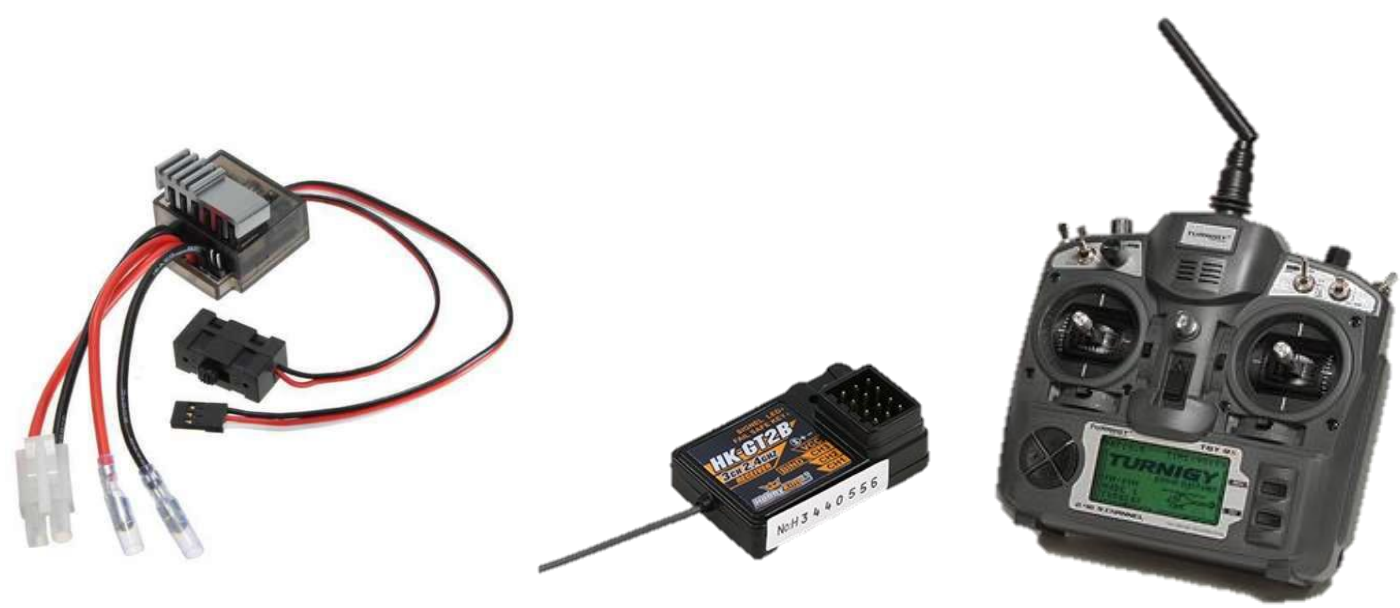

Figure 38: The speed controller, receiver, and transmitter used in the control system

The prototype vehicle is designed to be a proof-of-concept for the combined locomotion and adhesion vacuum method. Because of this, design of a control system is not within the scope of the project. In order to test the mobility of the prototype, hobby-grade remote control speed controllers and receivers $(\mathrm{RX})$ will be utilized to provide controllable power to the motors. The Turnigy $9 \mathrm{X}$, a hobbyist $2.4 \mathrm{GHz}$ transmitter (TX), will be bound to the receiver and configured to operate the left treadpod motors with the left stick and the right treadpod motors with the right stick. The choice of this specific hardware is due to its availability for use on this project. Any 
standard TX/RX pair with at least 2-channel capability can be substituted for the chosen components.

Table 18: Control electronics specifications

\begin{tabular}{ll}
\hline Property & Value \\
\hline Speed Controller Type & $320 \mathrm{~A}$ Brushed Speed Controller w/Reverse \\
Speed Controller Weight [lbf] & 0.11 \\
Speed Controller Cost, Each & $\$ 10.50$ \\
RX Type & HK-GT2B 3CH 2.4GHz Receiver \\
RX Weight [lbf] & 0.03 \\
RX Cost & $\$ 23.63$ \\
TX Type & Turnigy 9X \\
TX Cost & $\$ 69.64$ \\
\hline
\end{tabular}

\subsection{Secondary Component Design}

Several smaller components were also designed to add structure to the treadpods. The tensioner support bracket, round standoffs, and the shear support bracket all serve important functions, but are not sophisticated enough to warrant independent examination. The relative locations of each of these parts can be seen in previous sections, and specifically in Figure 25. The primary purpose of these parts is to hold the inside width of the treadpod at 1.32 in., allowing 0.070 in. of clearance from the 1.25 in. wide belts and pulleys. This clearance also allows the addition of PTFE wear surfaces on either internal surface if belt rubbing becomes an issue.
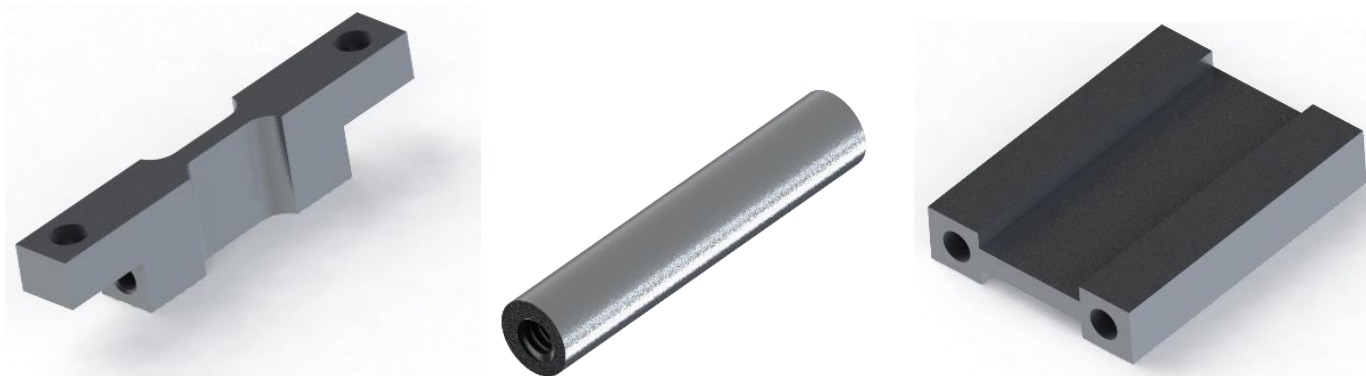

Figure 39: Renders of the secondary machined components 
Table 19: Secondary component specifications

\begin{tabular}{llll}
\hline Property & Tensioner Support & Round Standoff & Shear Support \\
\hline Material & 6061-T6 & 6061-T6 & 6061-T6 \\
Type & Billet Machined & Off-the-Shelf, Modified & Billet Machined \\
Width [in.] & 1.32 & 1.32 & 1.32 \\
Quantity Per Side & 1 & 4 & 2 \\
Weight Per Side [lbf] & 0.02 & 0.02 & 0.04 \\
\hline
\end{tabular}

While the round standoffs are easy to modify and simple to install, they are not ideal to resist shear between the two parallel treadpod frame pieces. In order to solve this problem, the two shear support brackets on each treadpod, shown on the right side of Figure 39, were designed and positioned orthogonally to each other in order to serve as the structural backbone of the treadpods, fixing the relative positions of the treadpod frame pieces.

\subsection{Full Assembly}

All components were modeled in Dassault Systemes' Solidworks. Proper material properties were applied to machined parts, and off-the-shelf parts were given appropriate masses. A full assembly model was created in Solidworks. All mechanical fasteners were included in the assembly in order to represent the mass of the vehicle as accurately as possible, as well as to verify assembly conditions and possible tool clearance issues. Not included, however, are motor wiring harnesses. The tube routing feature was used to simulate the tubing from the pressure manifold to the venturi pumps. The final assembly weight, according to Solidworks, is $6.41 \mathrm{lbf}$. A final, polished render is included below in Figure 40, and a wireframe drawing can be seen in Figure 41. 


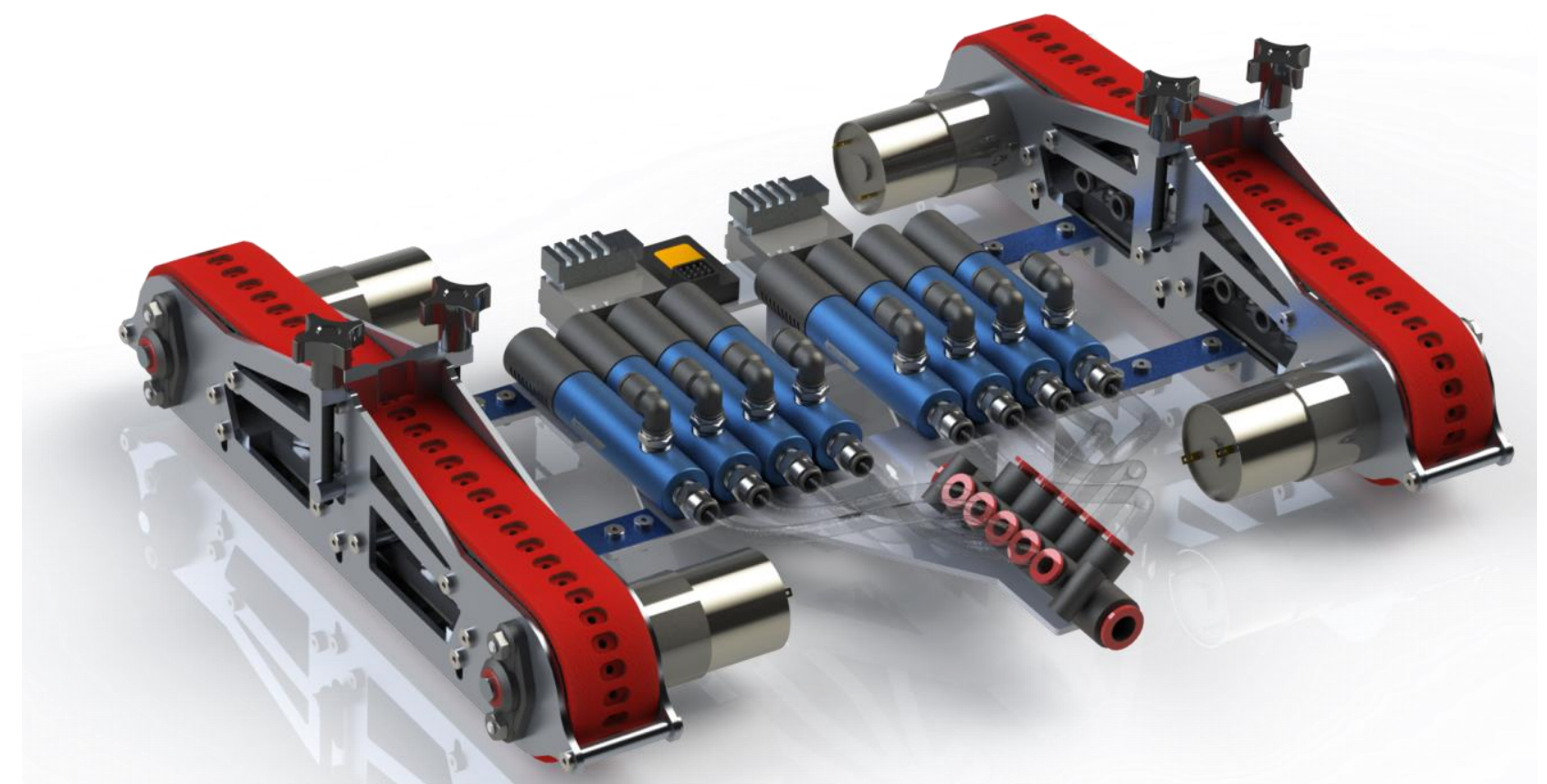

Figure 40: Full assembly, rendered in Solidworks

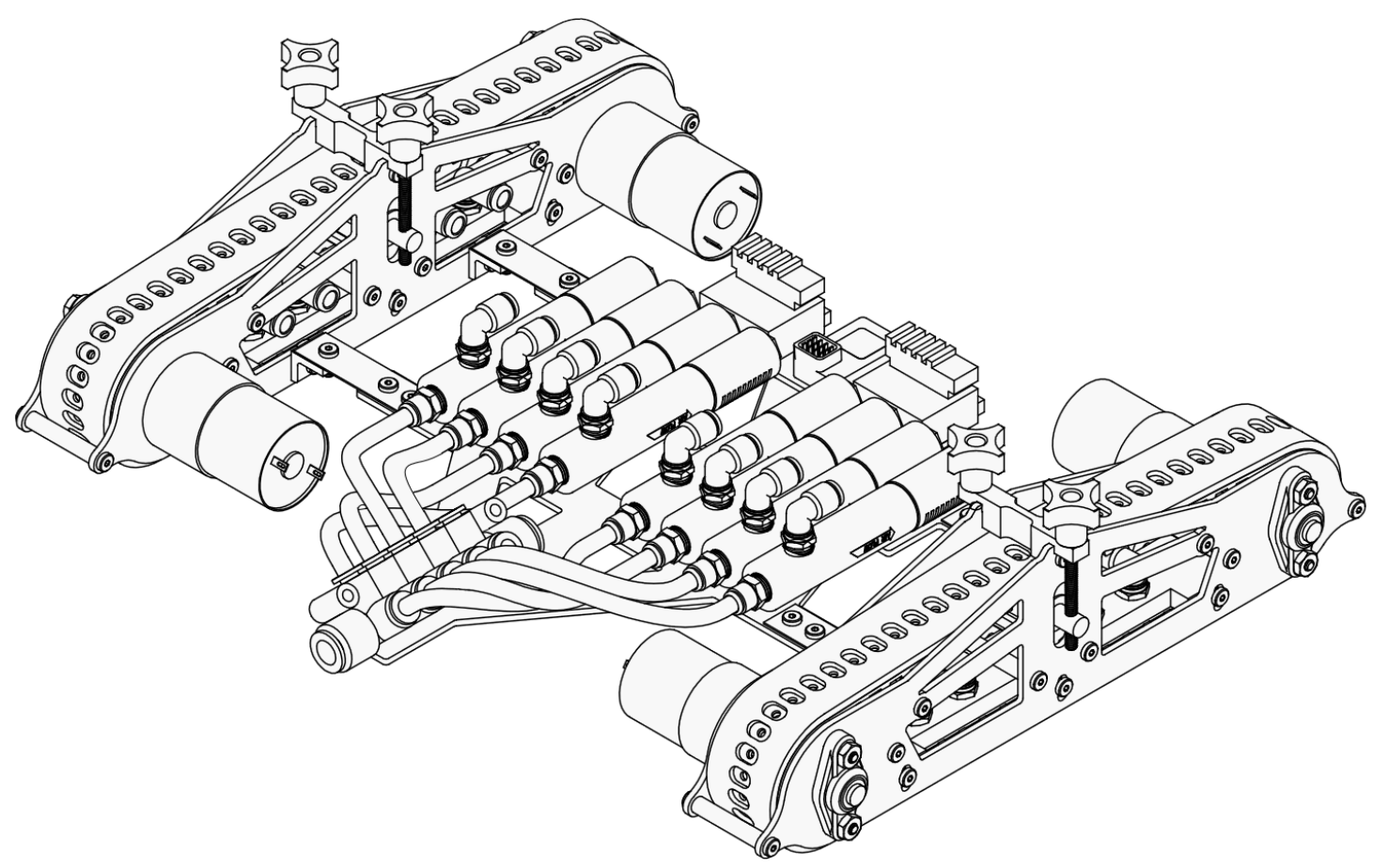

Figure 41: Full assembly, wireframe 


\section{MANUFACTURING AND PURCHASING}

Due to the prototype timeframe, manufacturing and requests for quotes were being performed in parallel with detail design for the vehicle. The following sections will overview some aspects of the part sourcing and ordering process, as well as the machining and production performed at Cal Poly.

\subsection{Bill of Materials}

Table 20: Bill of materials, broken down by subsystem

\begin{tabular}{ll}
\hline Subsystem & Subtotals \\
\hline Belt & $\$ 318.26$ \\
Pneumatic & $\$ 731.94$ \\
Motor, Drive and Tensioner & $\$ 217.63$ \\
Structural & $\$ 93.44$ \\
Control & $\$ 122.26$ \\
& \\
& Total \\
\hline
\end{tabular}

\begin{tabular}{lllll}
\hline Belt Subsystem & Vendor & Price & Qty & Total \\
\hline Neoprene 240L Belt, Linatex Backing & F.N. Sheppard & $\$ 156.00$ & 2 & $\$ 312.00$ \\
PTFE Tape, .5" Width & Amazon & $\$ 6.26$ & 1 & $\$ 6.26$ \\
& & & & \\
& & Subtotal & $\$ 318.26$ \\
\hline
\end{tabular}

\begin{tabular}{lllll}
\hline Pneumatic Subsystem & Vendor & Price & Qty & Total \\
\hline KM11-07-11-10 Push to Connect Manifold & eBay & $\$ 23.37$ & 1 & $\$ 23.37$ \\
1/4” OD Tubing, Roll & Ace Hardware & $\$ 6.99$ & 1 & $\$ 6.99$ \\
Push to Connect Fitting, Straight & McMaster Carr & $\$ 3.08$ & 8 & $\$ 24.64$ \\
JS-90-M-AA4 Miniature Venturi Pumps & Vaccon & $\$ 71.50$ & 8 & $\$ 572.00$ \\
Push to Connect Fitting, Elbow & McMaster Carr & $\$ 3.12$ & 16 & $\$ 49.92$ \\
6061-T6 Aluminum, .5”x1.25”x18 & Speedy Metals & $\$ 13.08$ & 1 & $\$ 13.08$ \\
PTFE Sheet, Adhesive Backed, 12"x12” & Amazon & $\$ 36.95$ & 1 & $\$ 36.95$ \\
EPDM Foam, Adhesive Backed, .5"Width & McMaster Carr & $\$ 4.99$ & 1 & $\$ 4.99$ \\
& & & & \\
& & & & \\
\end{tabular}

\begin{tabular}{lllll}
\hline Motor, Drive, and Tensioner Subsystems & Vendor & Price & Qty & Total \\
\hline 131:1 37D Metal Gearmotor & Pololu & $\$ 24.95$ & 4 & $\$ 99.80$ \\
Screw, Flat, M3-0.50 x 6mm, Pk50 & Grainger & $\$ 1.46$ & 1 & $\$ 1.46$ \\
L-Pitch, 16-Tooth Pulley Stock, Aluminum & CMTCo & $\$ 43.19$ & 1 & $\$ 43.19$ \\
\hline
\end{tabular}


Set Screw, \#8-32, 3/8" Length

Polymer Ball Bearing Flange, 5/16" ID

Screw, Flat, \#8-32 x .5"

Nut, Hex, \#8-32

L Pitch, 10-Tooth Pulley Stock, Delrin

Flanged Bearing, 5/16" ID

6061-T6 Aluminum Rod, .3125" Diameter

Steel Threaded Rod, \#8-32, 2" Length

Four-Arm Knob, \#8-32 Insert, 1" Diameter

6061-T6 Aluminum, .3125"x1"x12"
Ace Hardware

Grainger

Ace Hardware

Ace Hardware

CMTCo

Grainger

Grainger

McMaster Carr

McMaster Carr

Speedy Metals

$\begin{array}{lll}\$ 0.17 & 4 & \$ 0.68 \\ \$ 3.13 & 4 & \$ 12.52 \\ \$ 0.19 & 8 & \$ 1.52 \\ \$ 0.17 & 8 & \$ 1.36 \\ \$ 40.00 & 1 & \$ 40.00 \\ \$ 0.69 & 4 & \$ 2.74 \\ \$ 2.08 & 1 & \$ 2.08 \\ \$ 0.93 & 4 & \$ 3.72 \\ \$ 1.51 & 4 & \$ 6.04 \\ \$ 2.52 & 1 & \$ 2.52\end{array}$

Subtotal $\$ 217.63$

\begin{tabular}{lllll}
\hline Structural Subsystem & Vendor & Price & Qty & Total \\
\hline 6061-T6 Aluminum, .100"x12"x24" & Speedy Metals & $\$ 18.64$ & 1 & $\$ 18.64$ \\
6061-T6 Aluminum Angle, .5"x1", .125" Wall & McMaster Carr & $\$ 1.79$ & 1 & $\$ 1.79$ \\
6061-T6 Aluminum, .25"x1"x12"” & Speedy Metals & $\$ 1.68$ & 1 & $\$ 1.68$ \\
1095 Spring Steel, Hardened, Blued, 10, & McMaster Carr & $\$ 26.03$ & 1 & $\$ 26.03$ \\
Standoff, Aluminum, \#8-32x1.5", Pk10 & Grainger & $\$ 20.59$ & 1 & $\$ 20.59$ \\
Cable Tie Mounting Pads, 4-Way, Pk100 & Grainger & $\$ 9.38$ & 1 & $\$ 9.38$ \\
Cable Ties, Pk100 & Harbor Freight & $\$ 1.99$ & 1 & $\$ 1.99$ \\
Screw, Low Head, \#8-32 x 3/8" Pk50 & McMaster Carr & $\$ 6.67$ & 2 & $\$ 13.34$ \\
& & & & \\
& & Subtotal & $\$ 93.44$ \\
\hline
\end{tabular}

\begin{tabular}{lllll}
\hline & Vendor & Price & Qty & Total \\
\hline Transmitter, Turnigy 9X 2.4 GHz & Hobbyking & $\$ 69.64$ & 1 & $\$ 69.64$ \\
Receiver, HK-GT2B 2.4 GHz, 3 Channel & Hobbyking & $\$ 23.63$ & 1 & $\$ 23.63$ \\
Speed Controller, 320A DC w/ Reverse & eBay & $\$ 10.50$ & 2 & $\$ 21.00$ \\
Motor Wire & -- & - & - & -- \\
Connectors, Deans Style T-Plug, Pk20 & Amazon & $\$ 7.99$ & 1 & $\$ 7.99$ \\
& & & & \multicolumn{2}{c}{ Subtotal } & $\$ 122.26$ \\
\hline
\end{tabular}

\subsection{Tread Purchasing}

Requests for quote for the drive belts were submitted to both F.N. Sheppard and Gates Mectrol, both manufacturers of custom timing belts with similar capabilities. Gates Mectrol responded with No Quote, as they do not deal in small quantities to private parties. F.N. Sheppard returned with a quote, and after some discussion with the representative, provided two options, as outlined below in Table 21 . 
Table 21: Custom timing belt quotations

\begin{tabular}{lll}
\hline Property & Option 1 & Option 2 \\
\hline Belt Backing Material & Seamless, Overmolded Rubber & Cut and Bonded Linatex \\
Lead Time & 6 Weeks & 4 Weeks \\
Minimum Quantity & 6 Belts & 2 Belts \\
Price Per Belt & $\$ 105$ & $\$ 156$ \\
\hline
\end{tabular}

Despite the lower price per belt and seamless backing offered by the original option, the shorter lead time and lower minimum order quantity led to the selection of Option 2. The F.N. Sheppard representative claimed that the seam that would be present on the Linatex backing would be ground flat and would not cause any hardness or height discontinuities as the belt ran. Additionally, while the representative also stated that the overmolded rubber backing was comparable to Linatex, he noted that customers occasionally claim that the friction characteristics are slightly worse than brand-name Linatex. The order was placed on 4/18/2017. During the course of the production, F.N. Sheppard sent images of the belt in progress, reprinted in Figure 42.

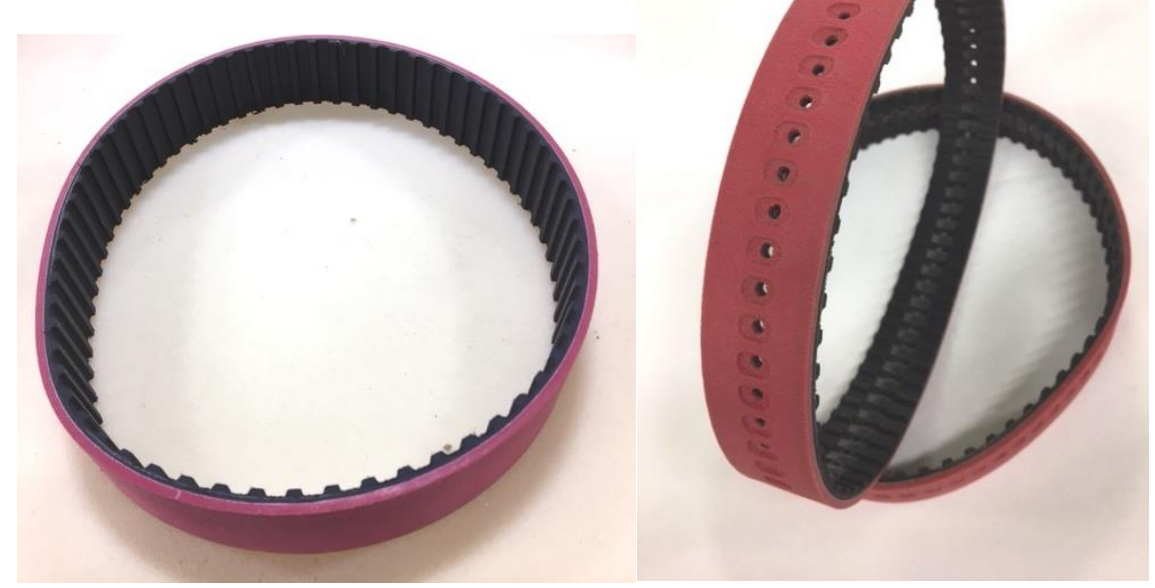

Figure 42: In-progress images of the belts provided by F.N. Sheppard

The completed belts arrived on 5/22/2017, approximately 5 weeks from the date of order. Initial inspection showed quality work on the counter-bore and perforation operations. However, 
the region of removed teeth, which was promised to be smooth to allow for a vacuum seal, instead had layers of fabric across the belt between each tooth. This discontinuous surface roughness led to the troubleshooting steps outlined in later sections, as sealing attempts with asreceived belts resulted in failure.

\subsection{Vacuum Manifold Manufacturing}

Using 6061-T6 aluminum bar stock, two vacuum manifolds were cut to net shape on a manual Bridgeport milling machine. After center drilling to ensure positional accuracy, the six \#8-32 tapped screw holes and the four 1/8-27 NPT pneumatic connections were drilled out to pilot diameter. A hand tapping machine was used to cut the treads in all holes to remove concerns of tapping off-axis.

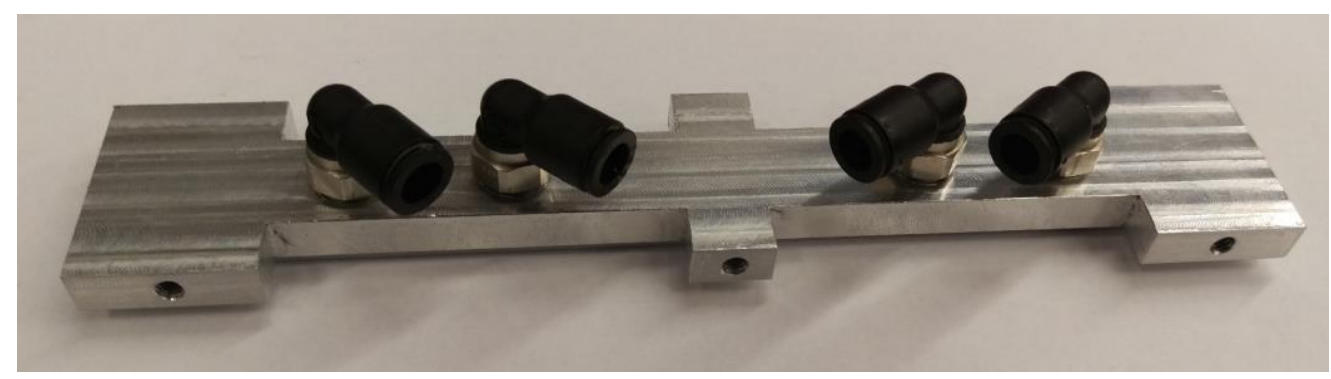

Figure 43: Top view of the vacuum manifold, assembled

In order to accurately cut the PTFE pieces to apply to the vacuum manifold, a $\mathrm{CO}_{2}$ laser cutter/engraver was used. PTFE is an excellent absorber of the $10.6 \mu \mathrm{m}$ wavelength light emitted by the $\mathrm{CO}_{2}$ laser tube, and so cuts very cleanly and without smoke or flare-up risk. The $3 \mathrm{M}$ 300LSE pressure sensitive adhesive on the back of the PTFE sheets did not seem to be negatively affected by the laser process. The PTFE was carefully applied to the manifold as seen in Figure 44. 


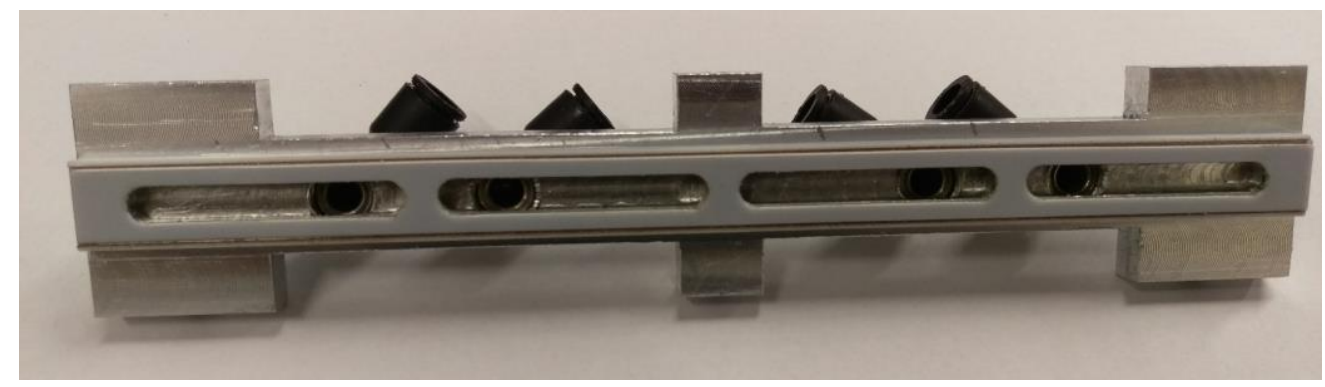

Figure 44: Bottom view of the vacuum manifold, assembled

Due to the narrow thickness of the vacuum manifold as well as the fact the that pilot holes for the 1/8-27 NPT fittings are not through holes, some creativity was required in order to allow full thread engagement on the pneumatic fittings. A standard tapered 1/8-27 NPT tap would bottom out in the hole before cutting threads deep enough for the fitting to be fully seated into the part. To solve this, a second 1/8-27 NPT tap was purchased and carefully cut using a fine abrasive wheel. Using this new, custom bottoming tap after an unmodified tap allowed the threads to be cut deep enough for full thread engagement on the pneumatic fittings.

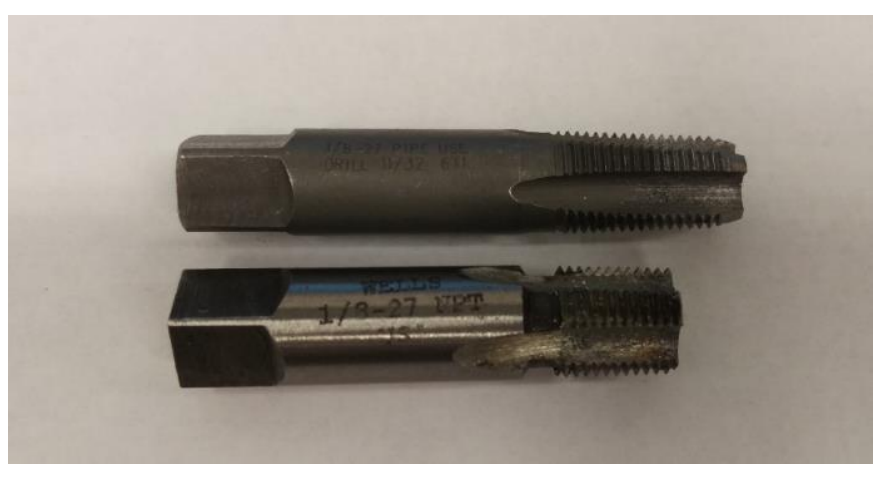

Figure 45: Standard and custom 1/8-27 NPT taps 


\subsection{Motor Subassembly Manufacturing}

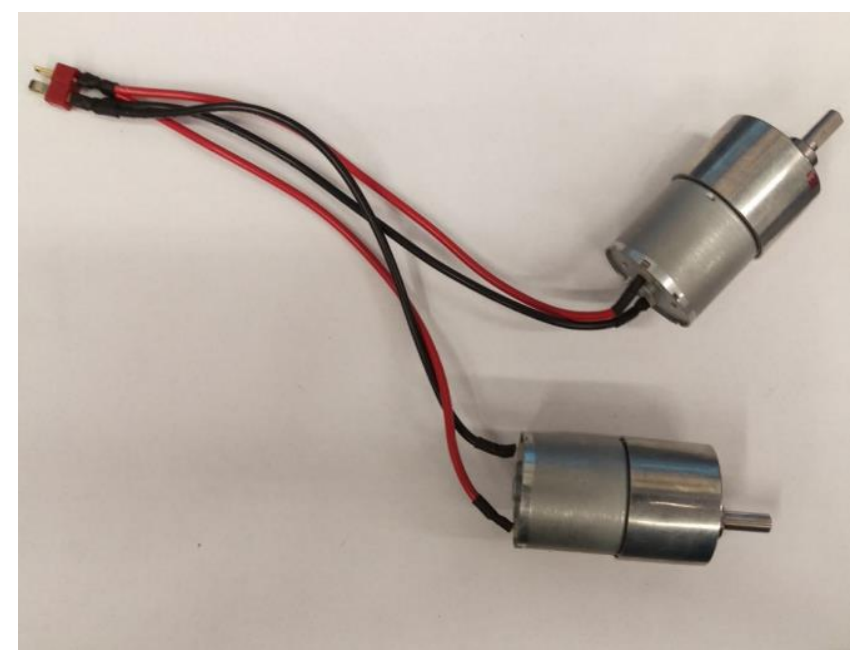

Figure 46: Assembled and soldered motor system, one side

Four 131:1 37D Metal Gearmotors were ordered from Pololu's website on 4/20/2017 and arrived less than a week later. The extent of the additional manufacturing required for the motor subsystem involved simple wiring and soldering. The motors were soldered in parallel to a single T-connector, with care to keep the exact same wire run length to each motor. This precaution is to keep the system resistance, and thus the voltage drops between the speed controller and each motor as similar as possible to maximize the chance that the motors will rotate in sync when a specified voltage is applied by the speed controller.

\subsection{Drive and Tensioner Pulley Manufacturing}

Quote requests for pulley stock for both the 16-tooth drive pulleys and the 10-tooth tensioner pulleys were sent to several manufacturers. The standard usable length of 8 in. for pulley stock is sufficient to make the required four drive pulleys, so one piece of each size of pulley stock was requested. 
Table 22: Timing belt stock quotations

\begin{tabular}{lllll}
\hline Supplier & Product & Price & Lead Time & Notes \\
\hline Illinois Pulley and Gear & 10T Plastic & $\$ 179.86$ & $1-2$ Weeks & Nylon available. \\
Illinois Pulley and Gear & 16T Aluminum & $\$ 165.89$ & $1-2$ Weeks & \\
Custom Machine Tool & 10T Plastic & $\$ 40.00$ & 1 Week & Delrin available. \\
Custom Machine Tool & 16T Aluminum & $\$ 43.19$ & 3 Days & \\
Onedrives US & 16T Aluminum & $\$ 90.00$ & 2 Weeks & \\
Onedrives US & 10T Aluminum & $\$ 46.00$ & 2 Weeks & Plastics not available. \\
\hline
\end{tabular}

Based on the quotes received one of each pulley stock size was ordered from Custom Machine Tool Corp on 4/18/2017 and the materials were received one week later.

Manufacturing of the drive pulleys required two manual lathe operations to turn the stub axle, bore the motor axle hole, and face the pulley to width. Two mill operations were performed using a vertical-axis rotary table to cut the radial weight reduction slots in both faces. Finally, a simple mill operation allowed the grub screw hole and tool access holes to be drilled and tapped. All four drive wheels were manufactured without incident.

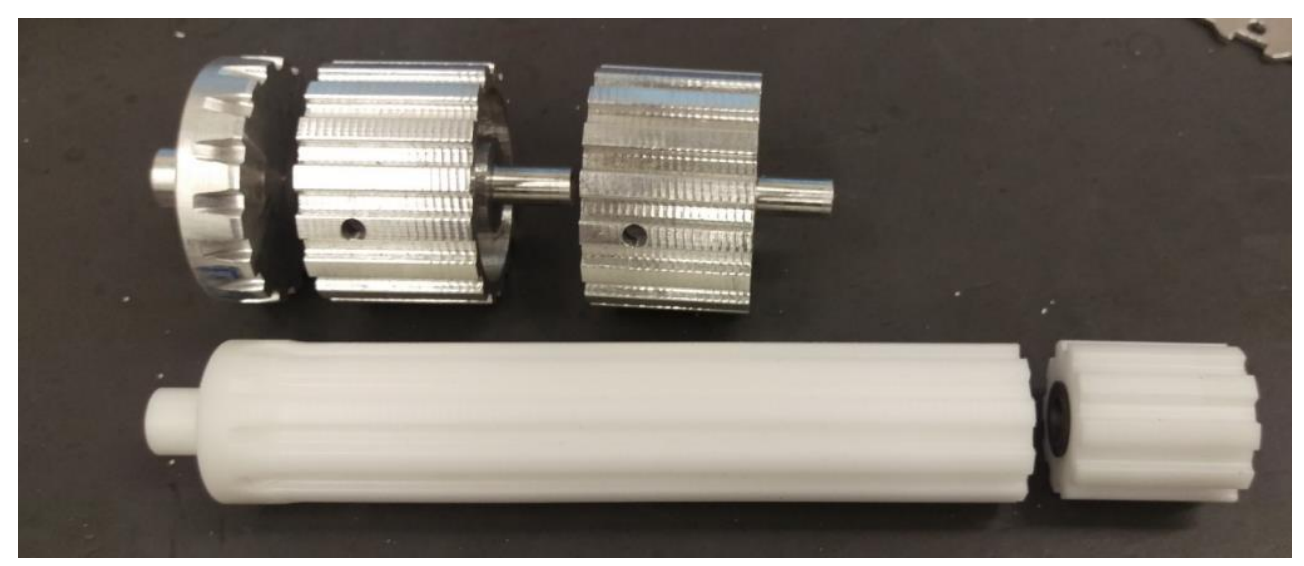

Figure 47: Remaining pulley stock with the machined parts for one treadpod

Tensioner pulley manufacturing consisted of two lathe operations to bore the tensioner axle hole, part off, and face to length. The polymer flange bearings were a light press fit into the pulley's center bores without requiring any adhesive. 


\subsection{Frame Manufacturing}

The majority of the manufacturing of the frame was performed using the Ward Kit water jet machine operated by the Industrial Technology and Packaging department at Cal Poly. To prepare the parts for cutting, configurations were made of both types of treadpod frame and center frame parts. These manufacturing configurations featured slightly undersized holes and removed countersink features. Water jet creates a tapered kerf, so in order to ensure cylindrical and properly dimensioned through holes and pilot holes, all holes were post-drilled to size on a drill press. All five parts (four treadpod frames and one central frame) were arranged to fit on a single $12 \times 24$ in. sheet of 0.100 in. thick 6061-T6 aluminum and were cut, as shown in Figure 48. The cutting time for a full set of parts came in at approximately nine minutes. Following water jet cutting, each part was thoroughly deburred, all holes were drilled to final dimensions, and any tapping and countersinking operations were performed.

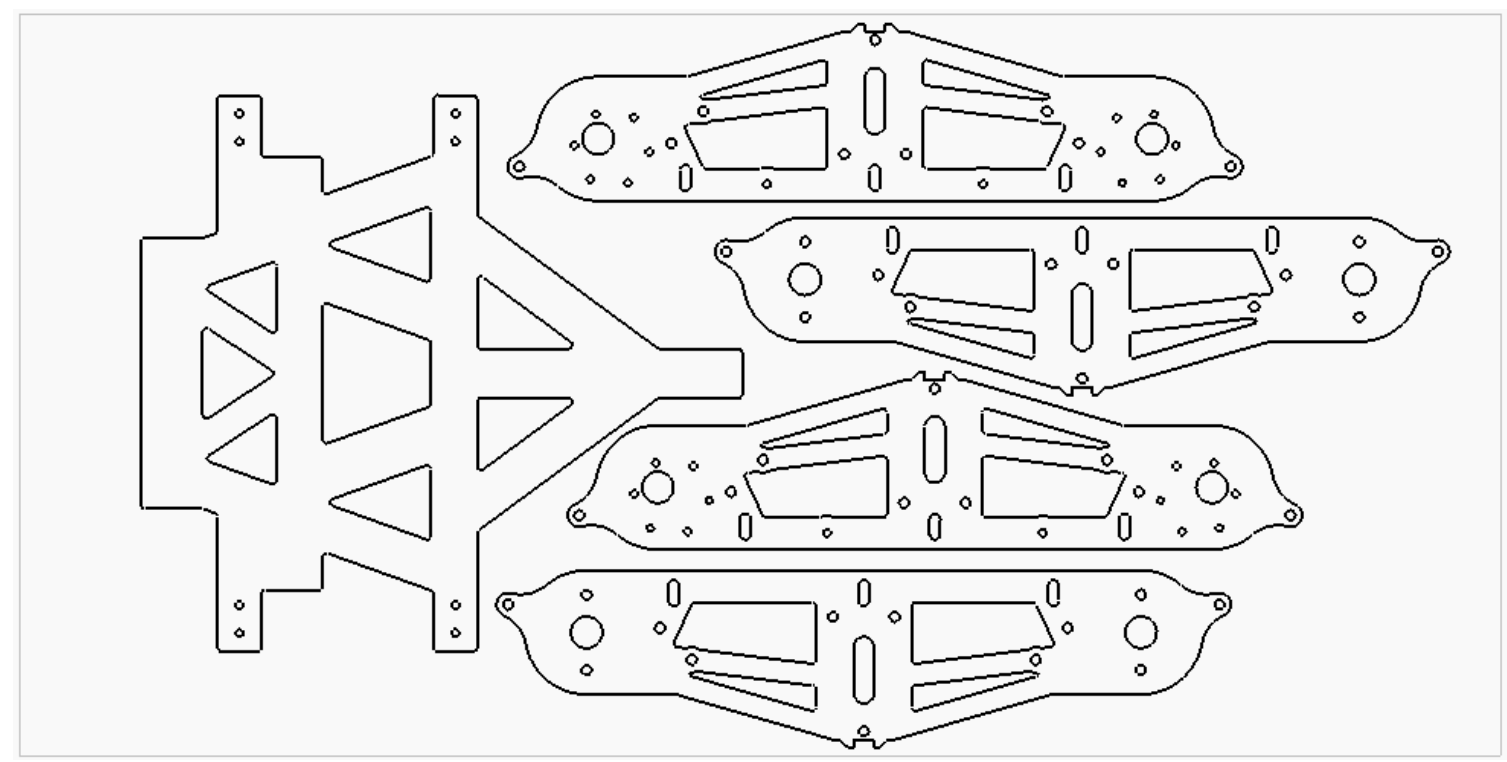

Figure 48: Nested parts ready for waterjet on a 12"x24" sheet

During the first water jet cutting operation, the stock shifted slightly during cutting, causing one of the motor-side frame pieces to be unusable. Additional $0.100 \mathrm{in}$. aluminum sheet 
was sourced locally and spare parts were cut during the next available opportunity. However, the local metal supplier carried the desired 0.100 in. thickness solely in 5052 alloy aluminum. Of comparable strength (and superior formability and corrosion resistance) to 6061-T6, the 5052 alloy is known to be less easily machined. However, as the only machining processes beyond water jet cutting are drilling and tapping, the 5052 alloy will not cause any problems when used as replacement part material.

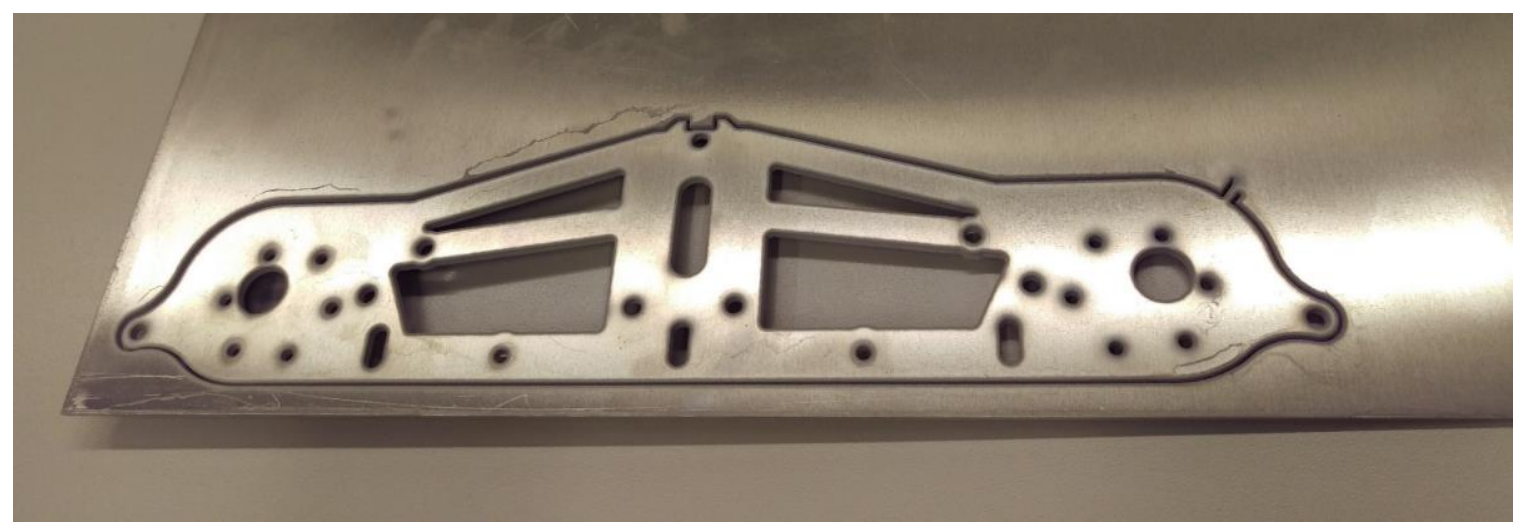

Figure 49: Water jet cut frame piece, motor-side

\subsection{Suspension Flexure Manufacturing}

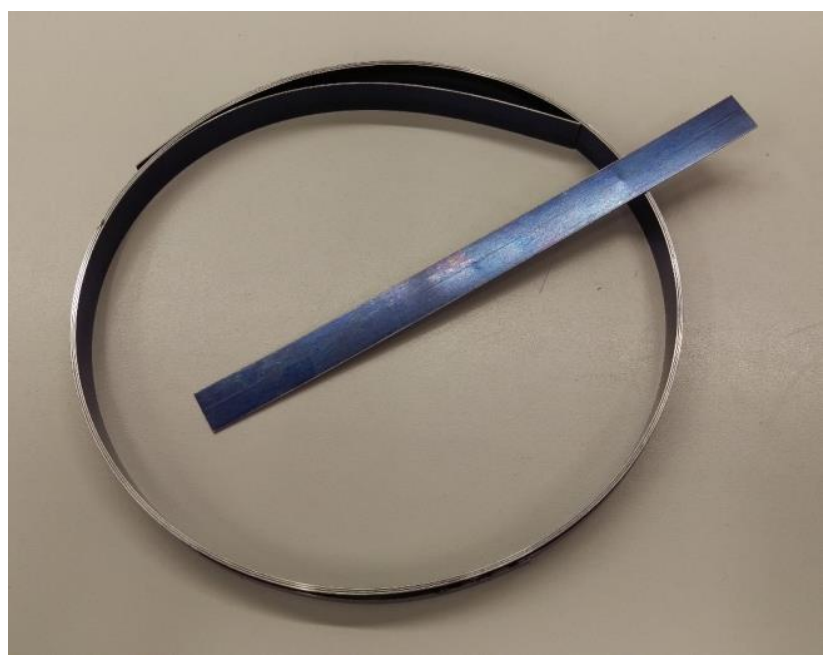

Figure 50: 1095 spring steel coil, as received 
A 10 foot long roll of 0.025 in. thick, 0.625 in. wide 1095 spring steel was purchased from McMaster-Carr to serve as the manufacturing blanks for the suspension flexures. The spring steel suspension flanges were machined using a manual mill to precisely space the attachment holes, and were then cut to length with sheet metal shears. When possible, the spring steel should be formed with punches and shears, as the hardness of the material makes drilling or conventional cutting a labor-intensive and messy process.

\subsection{Secondary Component Manufacturing}

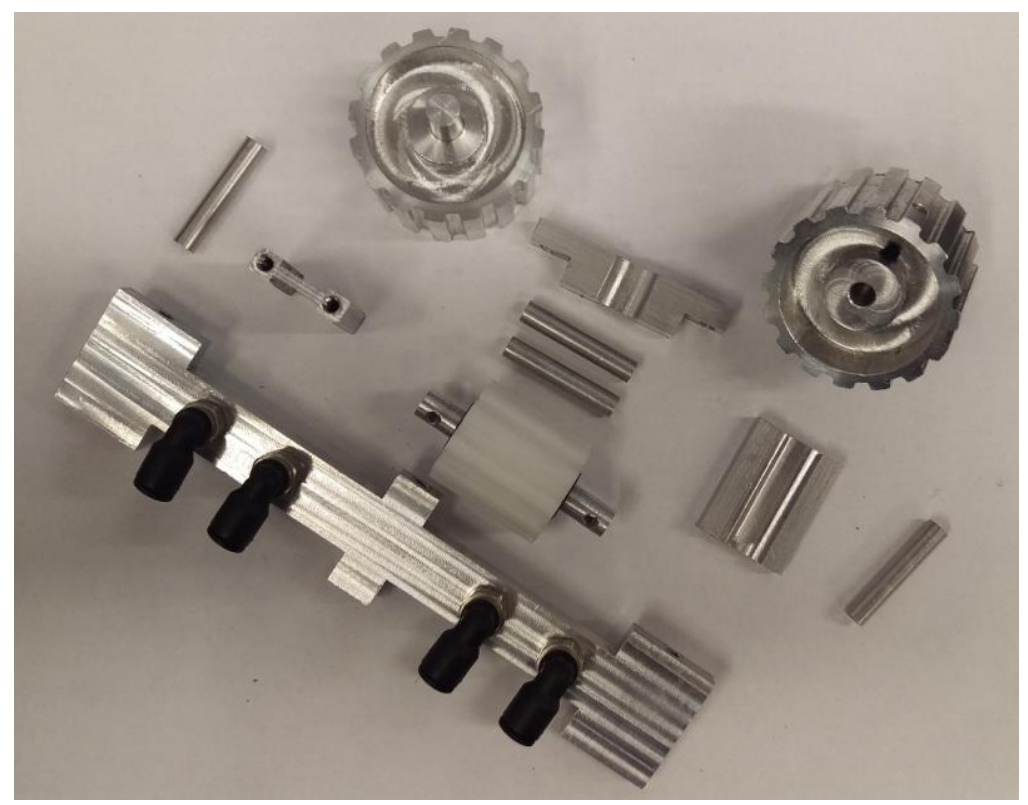

Figure 51: All machined parts for one treadpod

The remaining components in the assembly, including the tensioner bracket, round standoffs, idler axle, and shear support bracket, were manufactured without incident using manual mills and lathes. Figure 51 shows all internal components present in each tread pod. 


\subsection{Full Assembly}

Following manufacture, all parts were assembled. With the exception of the flat-head M3 screws required by the motors, all fasteners on the vehicle were designed to be \#8-32 for ease of service and assembly. Additionally, the only hex nuts in the assembly are used to affix the drive pulley support bushings. All other fasteners are threaded into tapped holes, reducing the number of tools needed for assembly or disassembly.

After cleaning the frame with mineral spirits, adhesive cable tie mounts were applied and all eight venturis, the pressure manifold, and the electronics were affixed using cable ties. Pneumatic tubing was routed from the first eight outlets of the pressure manifold to the eight venturi pumps. A hot water bath was used to slightly soften the tubing to allow for easier routing. Additional tubing was routed from each venturi pump's vacuum outlet to the eight push-toconnect fittings on the vacuum manifolds. Finally, all electrical connections were made between the speed controllers and receiver. Wherever possible, T-connectors were soldered into the wiring harness to facilitate maintenance. The result of the assembly is shown in Figure 52.

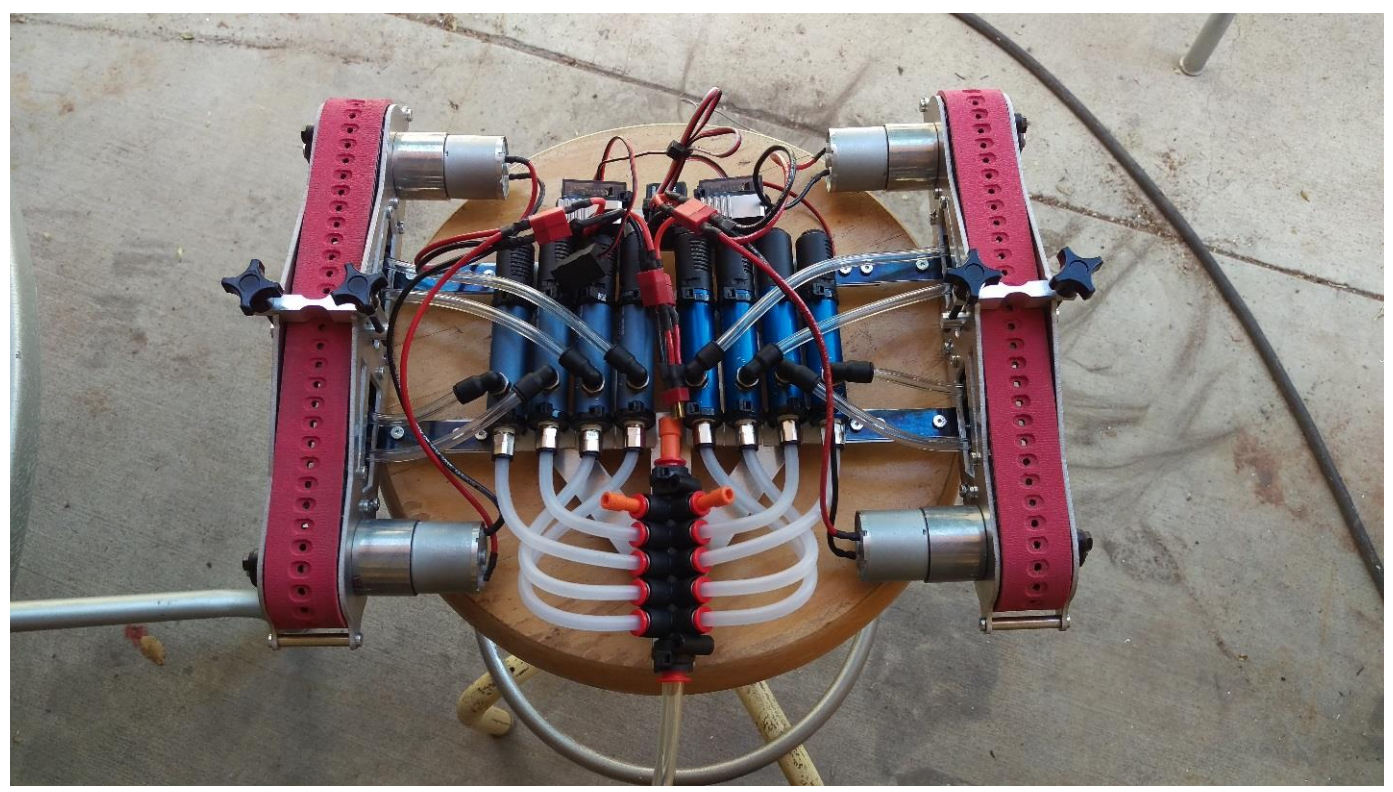

Figure 52: Photograph of the vehicle when first assembled 
Final assembly weight of 6.94 lbf fell below the design goal of $7.5 \mathrm{lbf}$. CG height (estimated in $\mathrm{CAD}$ ) was also well below the design criteria assumption. CAD estimate for the assembly weight was measured at $6.41 \mathrm{lbf}, 0.53 \mathrm{lbf}$ lower than the actual vehicle weight. This discrepancy is likely due to the fact that the venturi-to-treadpod tubing, cable tie mounts, cable ties, and electrical wiring were not modeled in the assembly. Vehicle footprint as well is also above the outlined max footprint in the design criteria. This overshoot, however, is not critical, as the vehicle is under both the weight and CG height goals.

Table 23: Final assembly metrics

\begin{tabular}{lll}
\hline Property & Design Criteria & Final Vehicle \\
\hline Weight [lbf] & 7.5 & 6.94 \\
CG Height [in.] & 3 & 1.55 (CAD Estimate) \\
Footprint [in. $x$ in.] & $12 \times 12$ & $15.6 \times 12.9$ \\
\hline
\end{tabular}




\section{TESTING AND TROUBLESHOOTING}

\subsection{Control System Test}

The first critical step towards a functioning prototype is to ensure that the control system functions properly and intuitively. For the simple system installed on the prototype, this involves binding the transmitter to the receiver on the vehicle, ensuring that the directions and channels of the left and right treadpod speed controllers are configured using the transmitter's user interface, and testing the basic locomotion of the vehicle.

The Turnigy 9X transmitter is best suited to flying vehicles, and as such some special configuration was required to provide a suitable and logical control scheme for the vehicle. The objective is to control the forward-back motion of the left treadpod on the left stick, and the right treadpod on the right stick. The majority of multirotor and helicopter control schemes in the Turnigy 9X's library map channels 1 and 4 to the left and right sticks. Using the lightweight, 3 channel receiver that is on the prototype, therefore, will require a workaround. Additionally, the left stick on a Mode 2 controller such as this $9 \mathrm{X}$ is almost always throttle, which maps the zero control input to the furthest down position of the stick. Required for this vehicle is a zero control output at center stick to allow for both forward and reverse motion.

Several "glider" control schemes use channels 3 and 2 for elevator and airbrake, respectively. Both of these control outputs are zero at center stick. Thus, the transmitter is configured for a glider, and the left-side speed controller is connected to channel 3 on the receiver, while the right-side speed controller is connected to channel 2. Once electrical connections were made, the receiver was bound to the transmitter, and the vehicle responded as desired. 


\subsection{Venturi Vacuum Test}

The first pneumatic test performed on the full vehicle was a simple venturi test. This test was designed to check for any major leaks, obstructions, or missed connections in the first stages of the pneumatic system. Input air at 80 psi was fed into the pressure manifold. An Ashcroft pressure/vacuum gauge was then connected to the output of each venturi pump. Pumps are numbered one to eight and from left to right as seen in Figure 53. As a reference, the maximum vacuum rated by Vaccon is $28 \mathrm{inHg}$, or 13.75 psi.

Table 24: Preliminary venturi pressure results

\begin{tabular}{lllllllll}
\hline Venturi & 1 & 2 & 3 & 4 & 5 & 6 & 7 & 8 \\
\hline Pressure [inHg] & 26.5 & 26.5 & 26.5 & 26.5 & 27.0 & 27.0 & 27.0 & 27.0 \\
Pressure [psi] & 13 & 13 & 13 & 13 & 13.3 & 13.3 & 13.3 & 13.3 \\
\hline
\end{tabular}

The results of this test were interpreted to mean that the vacuum pumps were functioning correctly and without any major leaks or other issues. The values read slightly below the rated maximum, but the discrepancy was small enough to possibly be attributed to gauge error, and was ignored.

It was discovered after the conclusion of testing, however, that the supply air pressure provided by the Cal Poly machine shops often did not exceed 60 psi, below the Vaccon pumps' rate input pressure of $80 \mathrm{psi}$. This lacking air pressure could have accounted for the small decrease in venturi pressure, and also could have caused a decrease in evacuation rate. However, as the error was discovered after the vehicle proved its capability using a lower input air pressure, it was not considered to be a critical break in test procedures. 


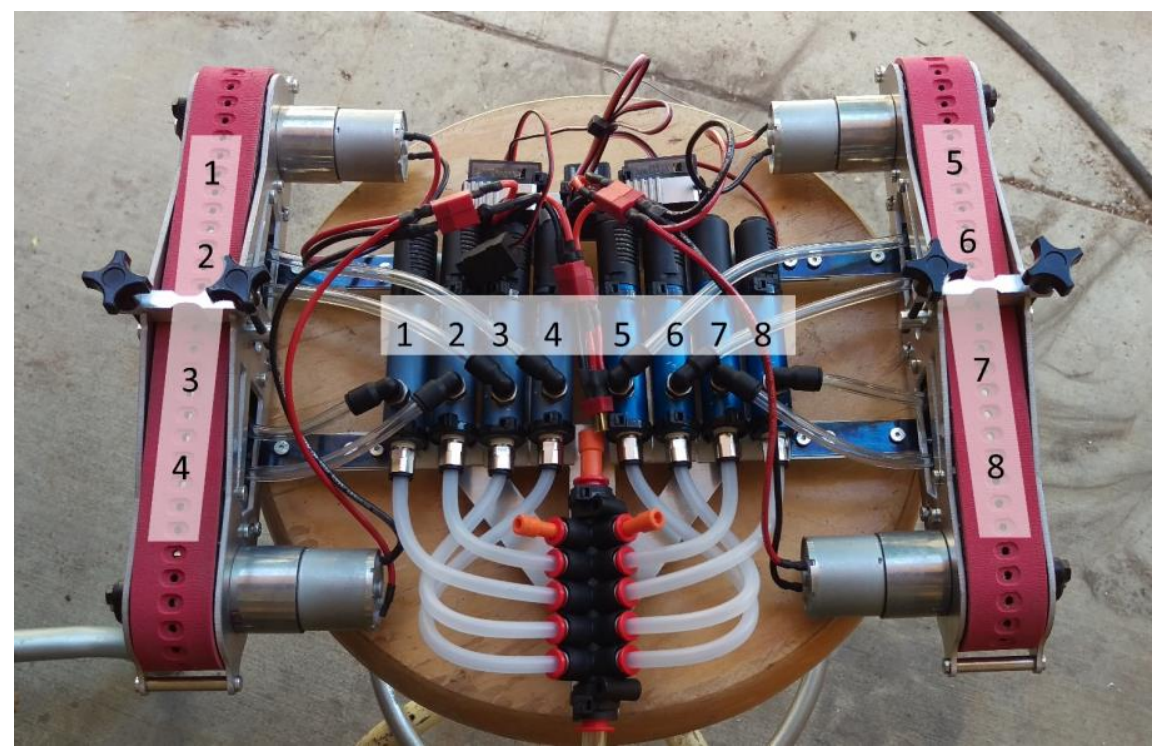

Figure 53: Full assembly, venturis and manifold sections numbered

\subsection{Manifold-to-Tread Seal Revisions}

The major functional concern with the prototype vehicle relates to the critical seal between the vacuum manifold and the center channel of the sliding treads. As received from F.N. Sheppard, the treads were not machined to a properly smooth running surface, despite assurances that it would. For this reason, a lengthy period of experimentation was used to iterate on the sealing surfaces in order to improve the performance of the vehicle.

Table 25: Seal iteration summary

\begin{tabular}{lllll}
\hline Iteration & Tread Surface & Manifold Surface & Adhesion & Notes \\
\hline 1 & As Received & Teflon & None & \\
2 & Sanded & Teflon & None & \\
3 & Teflon Tape & Teflon & Light, static & Failure with motion \\
4 & Teflon Tape & Cast Silicone Caulk & Good, static & Not durable \\
5 & Teflon Tape & Silicone Rubber & Good, mobile & High sliding friction. \\
6 & Teflon Tape & EPDM Foam & Good, mobile & Lower sliding friction. \\
\hline
\end{tabular}

As summarized in Table 25, six iterations of sealing surfaces were attempted before a satisfactory sealing solution was found. Due to the speed of iteration, the data collected during this process was qualitative and comparative. Each iteration is briefly explained below. 


\subsubsection{Iteration 1: As Received}

On receipt of the belts, it was clear that a reliable seal would be impossible without modification of the as-received sliding bed belt surface. The backing fabric present on the timing belt teeth was not removed in the central channel of the belt, resulting in strips of fabric alternating with strips of machined neoprene where the teeth were removed. No images exist of this state, but refer to Figure 54 for an approximate image of the belt state. Upon assembly and testing, insufficient adhesion was observed, and the vehicle would not adhere to a vertical glass surface.

\subsubsection{Iteration 2: Sanded Belt}

The next design iteration involved lightly sanding the central channel of the belts to remove the fabric burrs along the cut edges. Using increasing grits of sandpaper, 500 followed by 1000 , the surface of the belt was noticeably improved. The sanded surface is captured in Figure 54. As expected, this process did not alleviate sealing issues between the belt and the manifold, and no usable adhesion was created. Sanding, however, was still a critical step to allow for the subsequent iterations. 


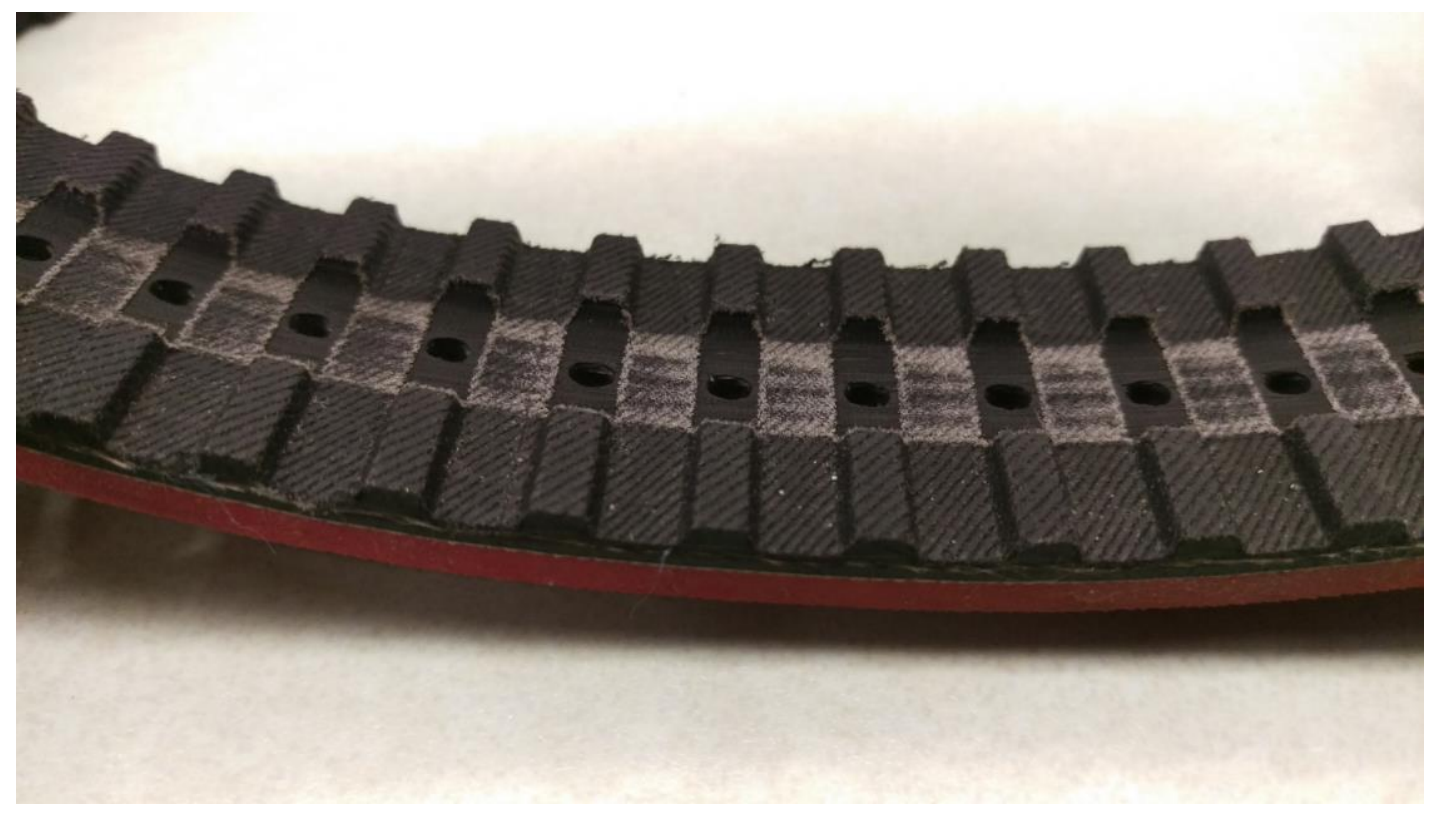

Figure 54: Belt central channel following sanding

\subsubsection{Iteration 3: Teflon Tape on Belt}

Thin Teflon tape was applied to the central channel of the belt. After application, the tape was punctured at each belt perforation, and the puncture was then widened with a punch that closely matched the through-hole diameter. While the tape did not smooth the surface of the belt to an extent which would allow for reliable sealing against the hard Teflon manifold seal, it would provide a slick running surface to allow for seal iterations on the manifold itself. After significant testing, the Teflon tape has proven to be remarkably durable, as it is adhered to a surface just several thousandths of an in. from the pitch line of the belt. Because of this, the length of the taped surface changes very little as it passes the drive and tensioner wheel radius. Thus, the tape does not bunch up or stretch out enough to delaminate from the belt itself. The few failures of the tape have been a result of the sliding friction against the manifold peeling up the free end of the tape. During testing, the addition of this taped surface allowed sufficient adhesion to hold the vehicle in some select orientations on a vertical glass surface. However, vehicle motion was still not possible. 


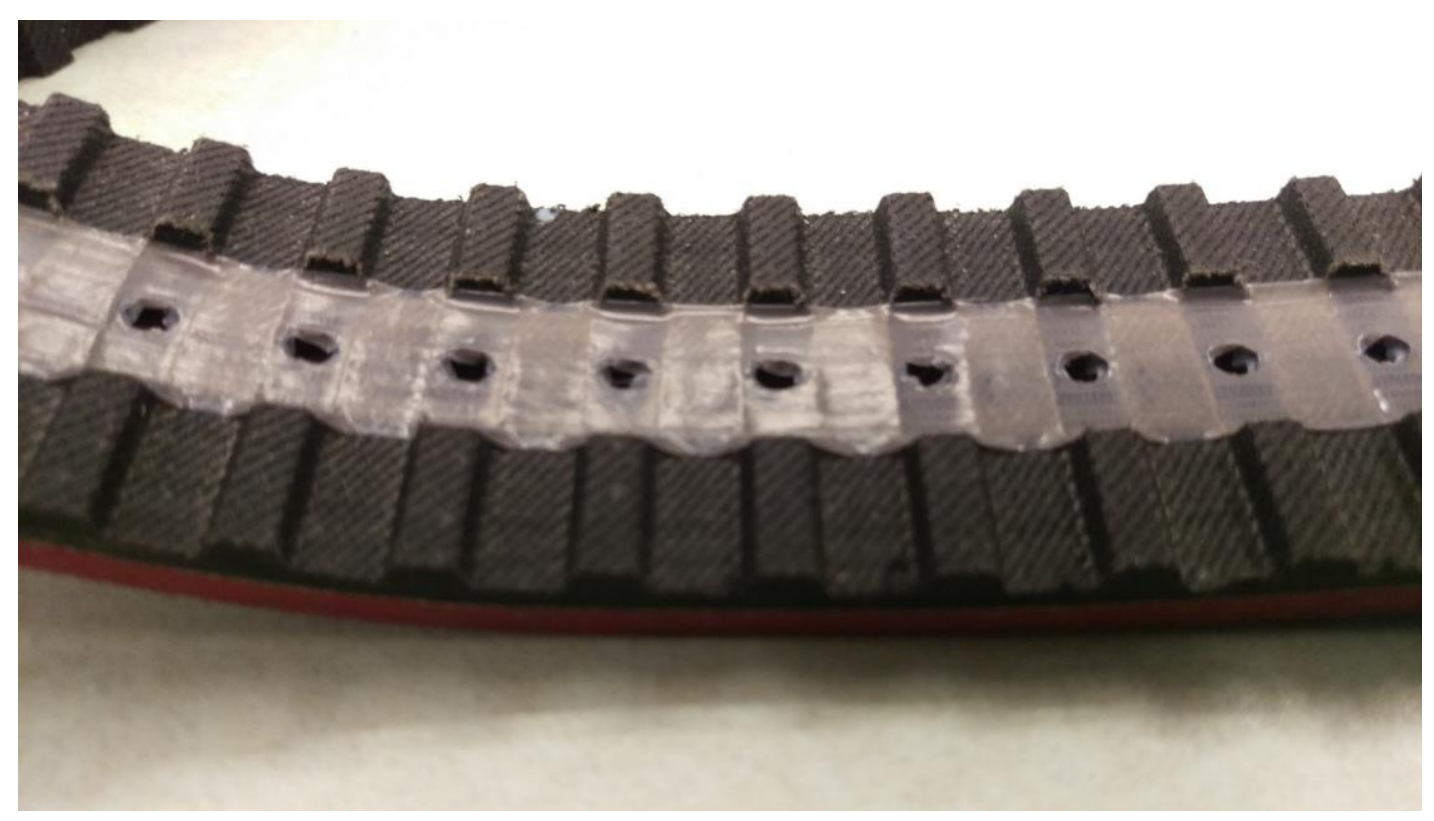

Figure 55: PTFE (Teflon) tape applied to inner surface of belt

\subsubsection{Iteration 4: Cast Silicone Caulking Manifold Seal}

As raw materials for the following two iterations were being shipped, a scrappy attempt was made to cast silicone calking to the aluminum surface of the vacuum manifold. The Teflon manifold seal was removed and the bonding surface was cleaned with mineral spirits. General Electric brand silicone weatherproofing caulk was applied generously to the surfaces of the manifolds. The manifolds were then inverted onto a flat sheet of Teflon in order to form the silicone caulk to a relatively thin, smooth layer. Following a 24-hour cure time, the manifolds were removed from the Teflon sheet and excess silicone was trimmed. The result of this process is shown in Figure 56. 


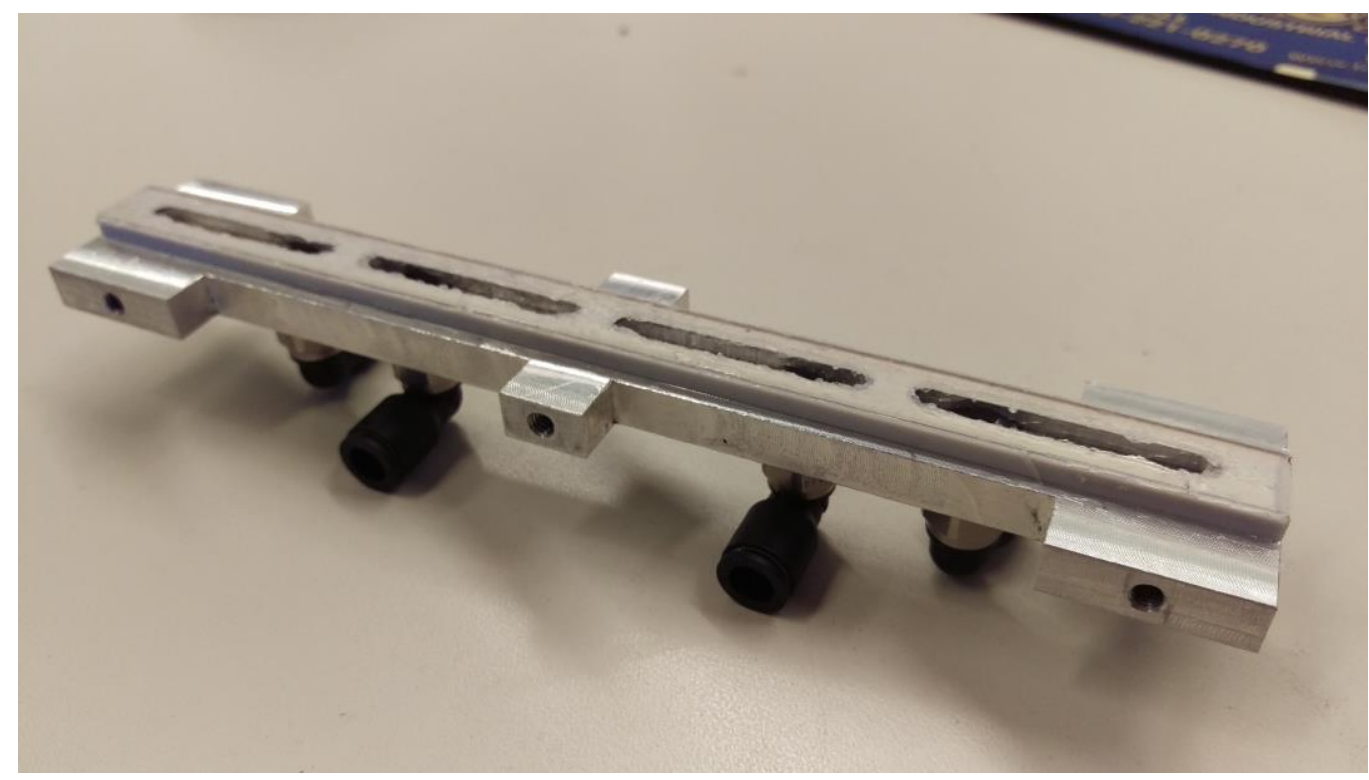

Figure 56: Cast silicone manifold seal

Both cast manifolds were assembled and the vehicle was connected to air supply.

Static adhesion proved to be very good. The vehicle held itself in any static position on a vertical glass surface. Some locomotion was possible, but the sliding friction between the belts and silicone manifold surfaces quickly damaged the soft silicone layer enough to break the seal, ending this test.

\subsubsection{Iteration 5: Silicone Rubber Manifold Seal}

Tested in parallel with the following iteration's EPDM foam, a 0.5-in.-wide, 1/16-in.thick roll of smooth-faced, adhesive-backed silicone rubber was purchased from McMaster Carr. Manifold sections were cut using the $\mathrm{CO}_{2}$ laser in the Cal Poly machine shops, and the silicone was adhered to the aluminum surface of the left vacuum manifold. The manifold, shown in Figure 57, was assembled into the vehicle during preparation for testing. 


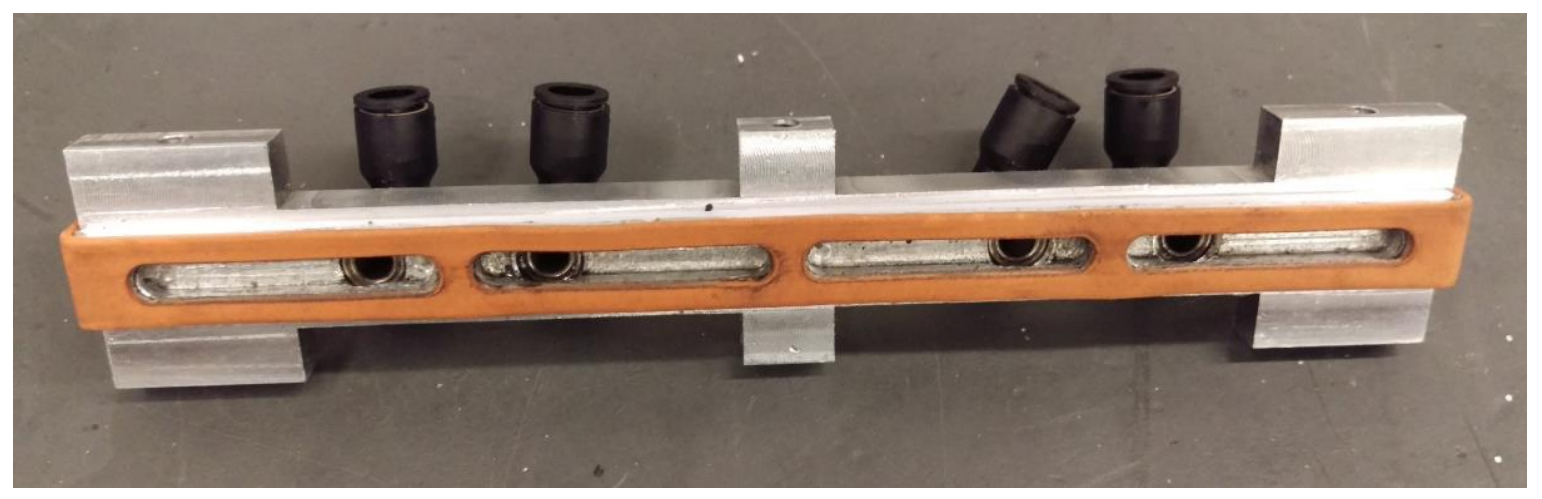

Figure 57: Silicone rubber manifold seal

\subsubsection{Iteration 6: EPDM Foam Manifold Seal}

Following the application of the silicone foam to the left manifold, a laser-cut section of 0.5-in.-wide, 1/16-in.-thick EPDM foam was similarly adhered to the right manifold. This EPDM foam is a closed cell, soft foam and is blended with neoprene and styrene-butadiene rubber (SBR) for enhanced abrasion resistance. The EPDM manifold is shown in Figure 58.

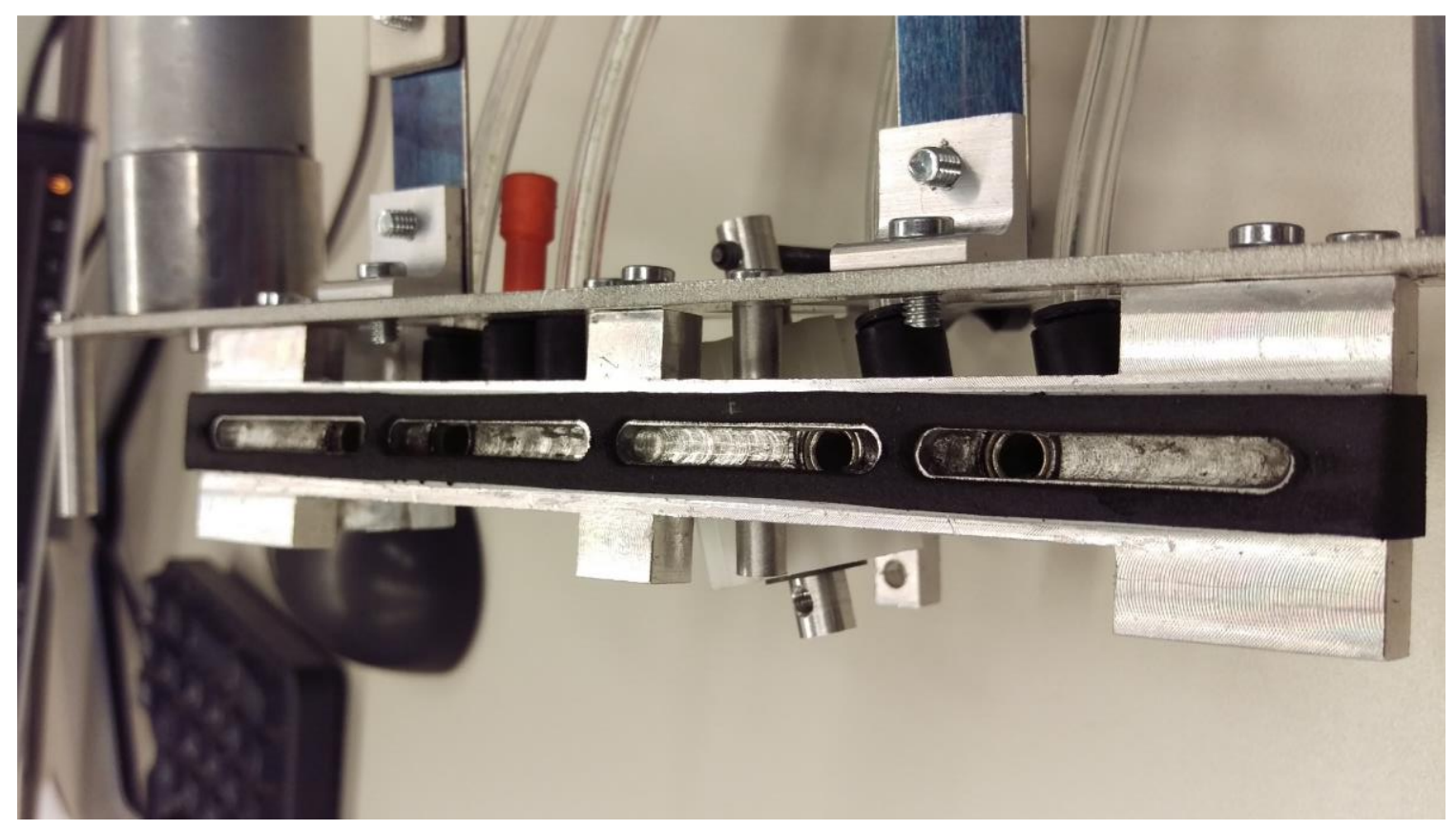

Figure 58: EPDM manifold seal 
Both manifolds were assembled into the vehicle and tested against a vertical glass surface. Vehicle adhesion was good and vehicle locomotion was possible. The climber was able to climb vertically both forward and reverse. Additionally, some turning and maneuvering was performed successfully. However, due to the limited space on the adjustable glass testing surface shown in Figure 59, full rotations of the vehicle were not possible.

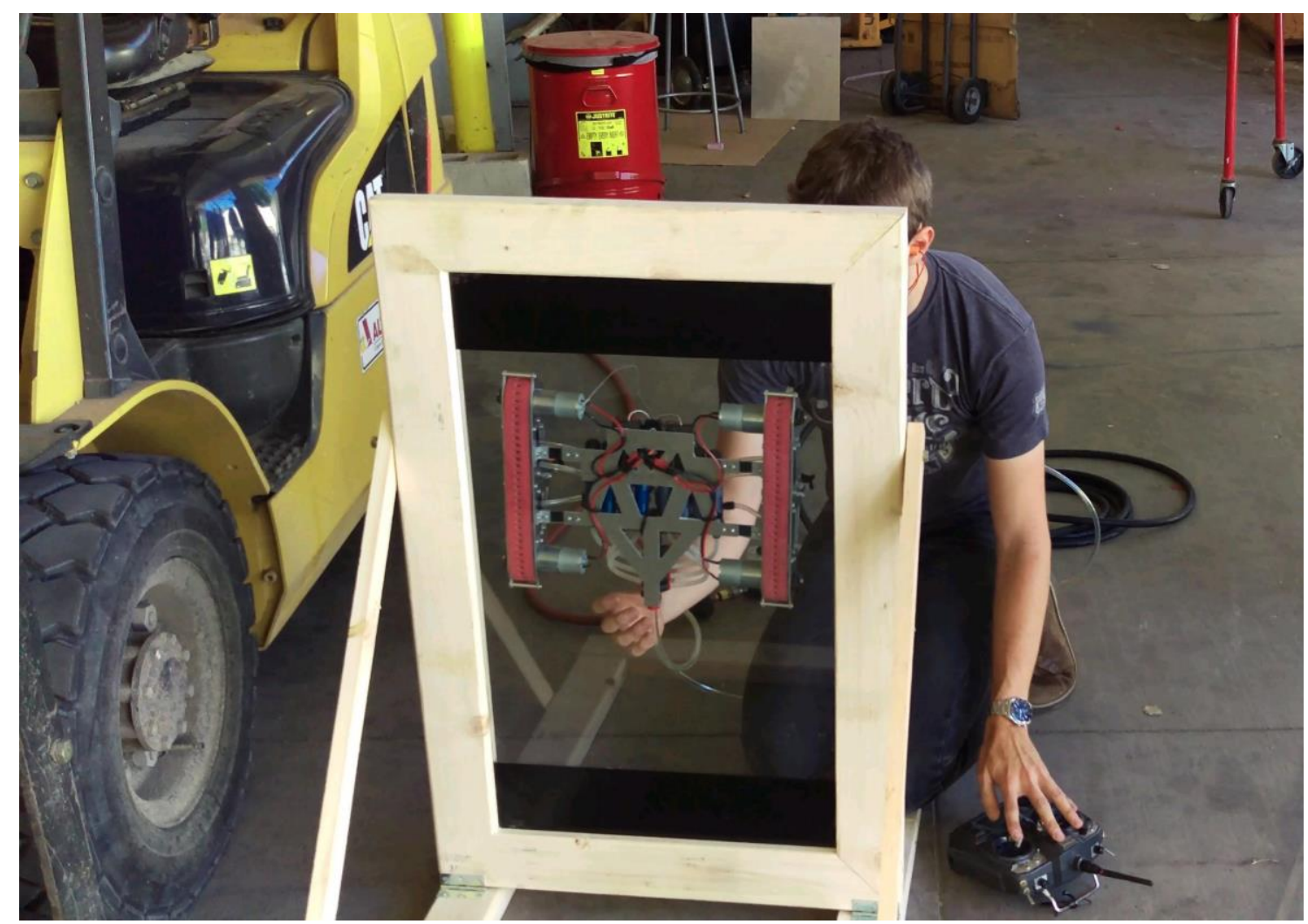

Figure 59: Still from video showing the first successful climbing test on adjustable glass surface

An informal qualitative comparison between the left tread (silicone rubber seal) and right tread (EPDM foam seal) was performed. The silicone foam seal appeared to more easily loose adhesion to the wall when the treadpod was rocked about its long axis. The likely explanation for this behavior is that the relative stiffness of the silicone rubber does not comply to the tread motion enough to allow for much misalignment angle. This, in addition to the significantly higher 
friction caused by the silicone rubber, led to a replacement of both manifold seals with EPDM foam seals. These seals would remain for the following testing cycles.

\subsection{Manifold Pressure and Pull Off Testing, Horizontal}

\subsubsection{Initial Testing}

In order to provide more detailed and qualitative testing, the single-inlet push-to-connect fittings were temporarily replaced with dual-inlet fittings shown in Figure 60. These fittings will allow for measurement of vacuum pressure inside the manifolds themselves, providing information on the actual seal and pressure status of each independent section. While not designed to use these fittings permanently, the frame pieces were designed to allow clearance for these larger, heavier fittings.

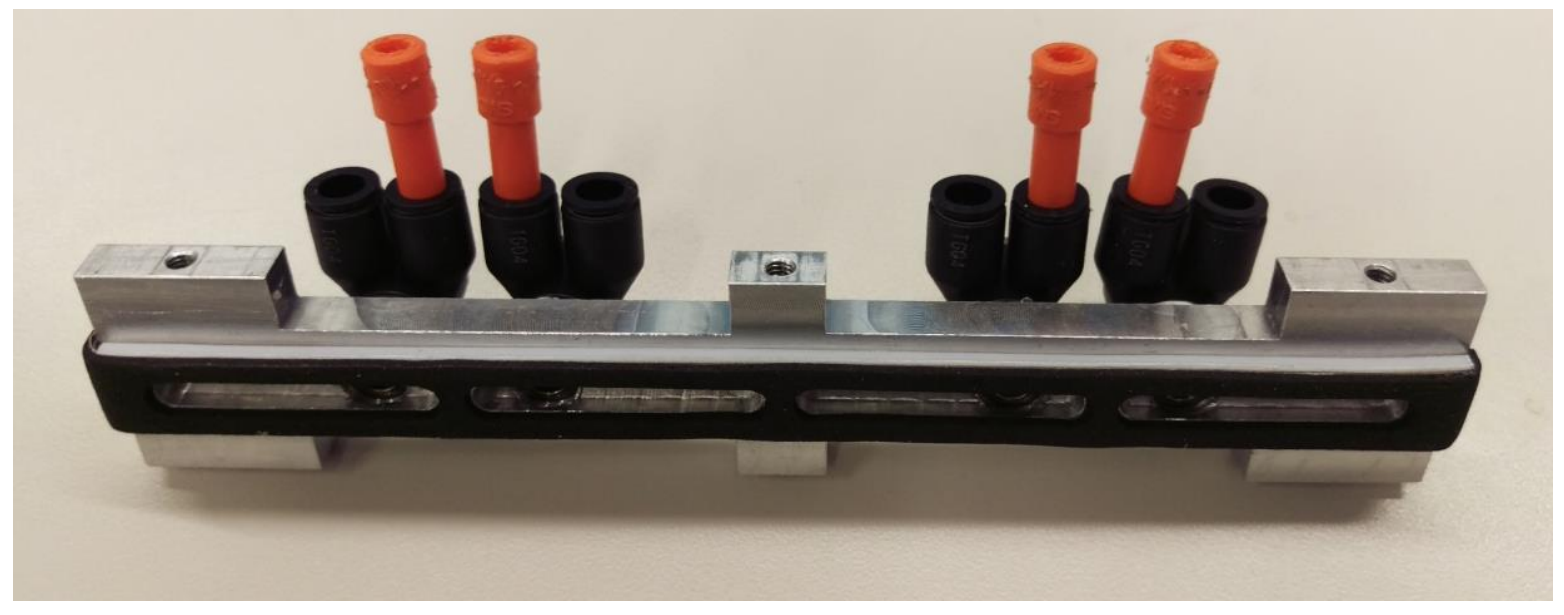

Figure 60: Manifold with dual-inlet pneumatic fittings installed

Using the adjustable glass testing surface in its horizontal orientation, vacuum pressure of each manifold section was independently measured using an Ashcroft pressure gauge. The results of these pressure readings are tabulated below, using the numbering convention laid out in Figure 53. 
Table 26: Initial manifold section pressure testing results

\begin{tabular}{lllllllll}
\hline $\begin{array}{l}\text { Manifold } \\
\text { Section }\end{array}$ & 1 & 2 & 3 & 4 & 5 & 6 & 7 & 8 \\
\hline Pressure [inHg] & 25 & 24.5 & 25.5 & 25 & 5 & 25 & 25.5 & 0 \\
Pressure [psi] & 12.3 & 12.0 & 12.5 & 12.3 & 2.4 & 12.3 & 12.5 & 0 \\
\hline
\end{tabular}

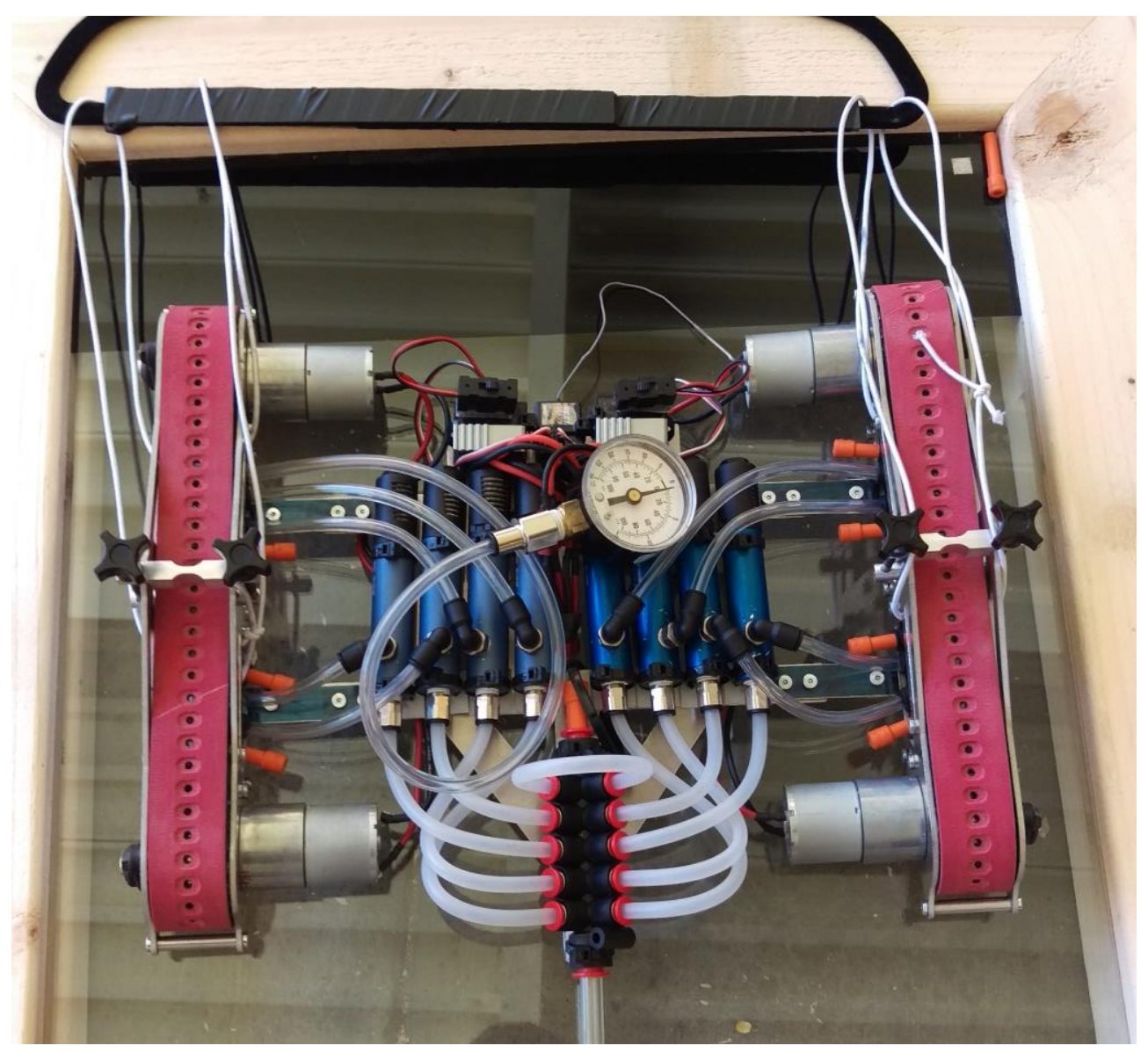

Figure 61: Manifold pressure testing setup on horizontal glass surface

Manifold sections 1 through 4, located on the left side treadpod, exhibited very good sealing. The pressure results were consistently and reliably achieved when the vehicle was picked up and replaced on the glass, showing a robust sealing solution may have been reached.

However, the right treadpod exhibited interesting behavior, failing to seal the first and last manifold sections. By rocking the treadpod slightly and applying a downward force, the seal was able to be achieved in the deficient manifold sections. This observation led to the hypothesis 
that a slightly warped suspension flexure may be leading to misalignment of the treadpod, resulting in the seal loss as the tread twists to meet the off-level manifold. This twisting could possibly affect both the first and last manifold sections. Stiffening the suspension could provide a solution to this problem. Additionally, if the manifolds themselves were adjusted too far downward as illustrated in Figure 63 and Figure 64, gaps may form at the front and back of each treadpod. These problems will be addressed before the next round of testing.

A pull-off test was performed despite the problems with the manifold seal. Using a simple support to ensure that both treadpods are pulled directly vertically, a digital spring scale was used to first weigh the vehicle and pull-off hardware and then, with the input air at $80 \mathrm{psi}$, pull the vehicle off of the horizontal glass surface. As expected, the resultant pull-off force was less than desired. The result is compared in Table 27 to both a calculated value taken from the manifold pressures in Table 26 as well as the desired pull-off force specified in the design criteria. Note that the calculation assumes that all seals remain unbroken and give way at the same time. Any leaks that develop during pulling will lower the max force seen before total vehicle pull-off.

Table 27: Initial pull-off force test results

\begin{tabular}{ll}
\hline Property & Value \\
\hline Weight [lbf] & 7.0 \\
Peak Pull-Off Force [lbf] & 18.5 \\
Total Vacuum Force [lbf] & 11.5 \\
Vacuum Force, Calculated from Pressures in Table 26 [lbf] & 26.2 \\
Difference from Calculation & $-56 \%$ \\
Design Criteria Vacuum Force from Table 2 [lbf] & 32.5 \\
Difference from Design Criteria & $-64 \%$ \\
\hline
\end{tabular}

\subsubsection{Revised Testing}

In order to solve several of the issues that were discovered in the previous testing, two major adjustments were implemented, as well as several minor fixes. First, 0.100 in. thick aluminum brackets replaced the spring steel suspension flanges. Thanks to the design of the 
suspension mounting to allow for this exact type of adjustment, this process was a drop-in replacement. The aluminum brackets were similar in width to the spring flanges and approximately $0.5 \mathrm{in}$. shorter. The result of this substitution is that the relative stiffness between the two treadpods is increased dramatically, keeping the treads flat on whatever surface they are resting. The limited compliance due to the 0.100 in. thick central frame span now dominates the system stiffness, providing sufficient suspension to overcome minor surface imperfections or manufacturing defects.

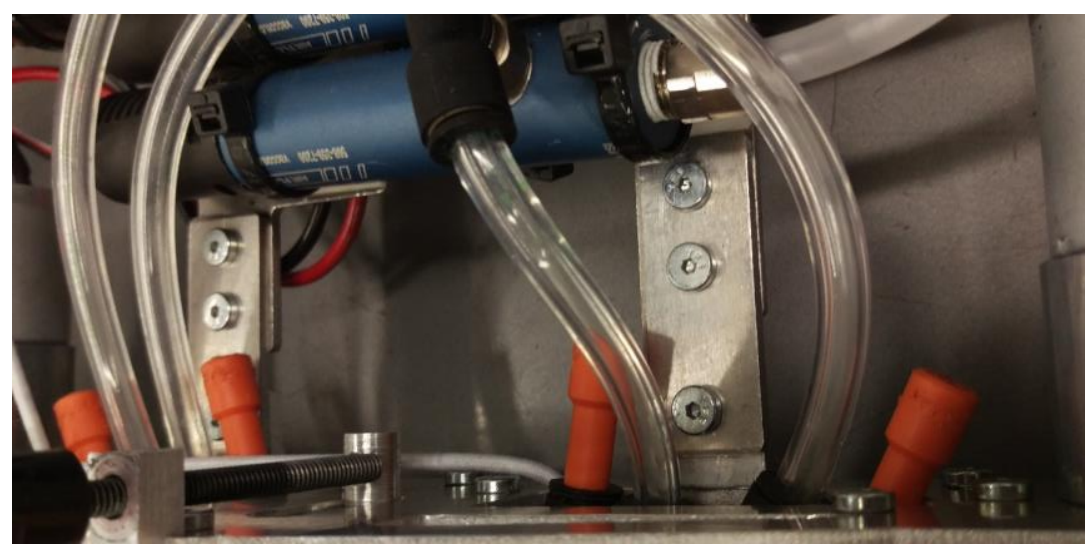

Figure 62: Replacement aluminum suspension brackets

The second adjustment ensured that the vacuum manifolds were correctly positioned in their adjustment slots such that the tread covering the full length of the manifold is sitting flat on the surface. At full height adjustment, the curve of the treads between the drive wheels and the manifold can cause gaps between the tread backing material and the driving surface. These states can be clearly seen in Figure 63 and Figure 64. By loosening six screws, the manifold can be slid to a more suitable position.

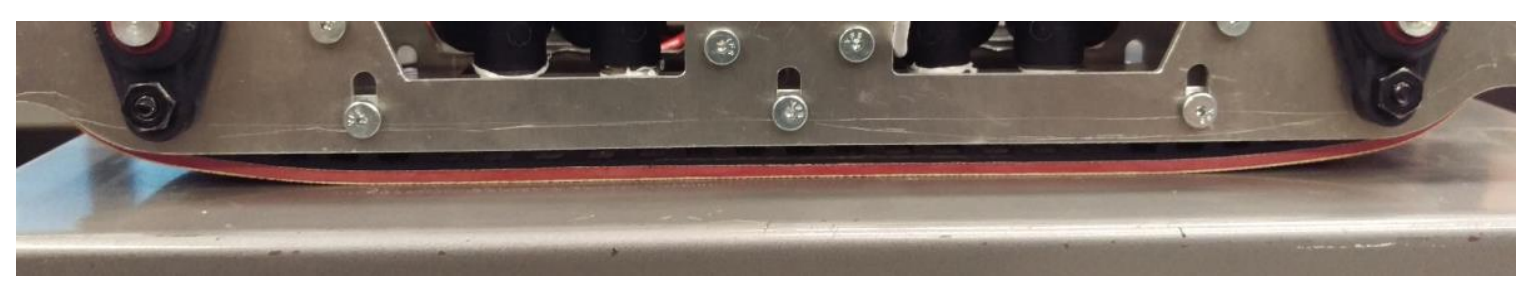

Figure 63: Manifold height excessive, causing gaps at either end 


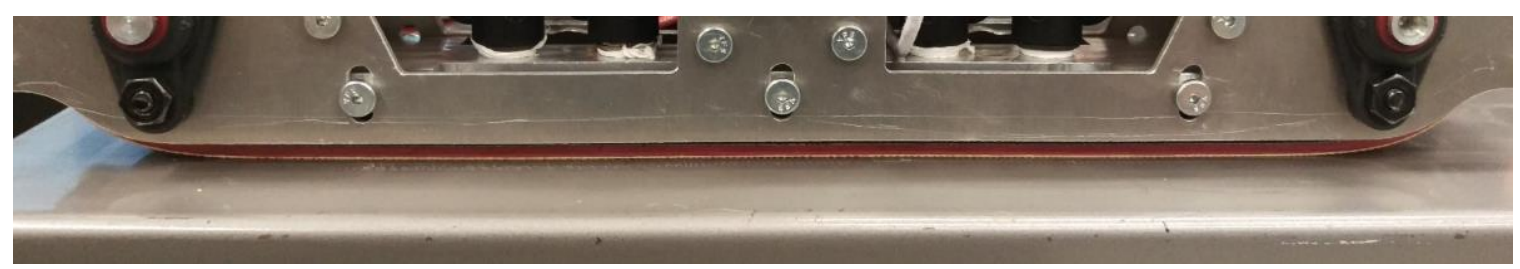

Figure 64: Manifod height properly adjusted, creating flush sealing surface

During disassembly, it was seen that the set screws on the drive wheels on the left treadpod had come loose, and the wheels were no longer well affixed to the motor shafts. This had caused the drive wheels to shift axially and rub on the PTFE tape applied around the ends of the frame to prevent unwanted wear caused by belt rub on the frame. The aluminum drive pulleys damaged the thin PTFE coating, as seen in Figure 65. During final reassembly of the vehicle, Loctite ${ }^{\circledR}$ thread locker was applied to all set screws, and the PTFE tape was removed and replaced. Further testing indicated no loosening of the set screws after this change.

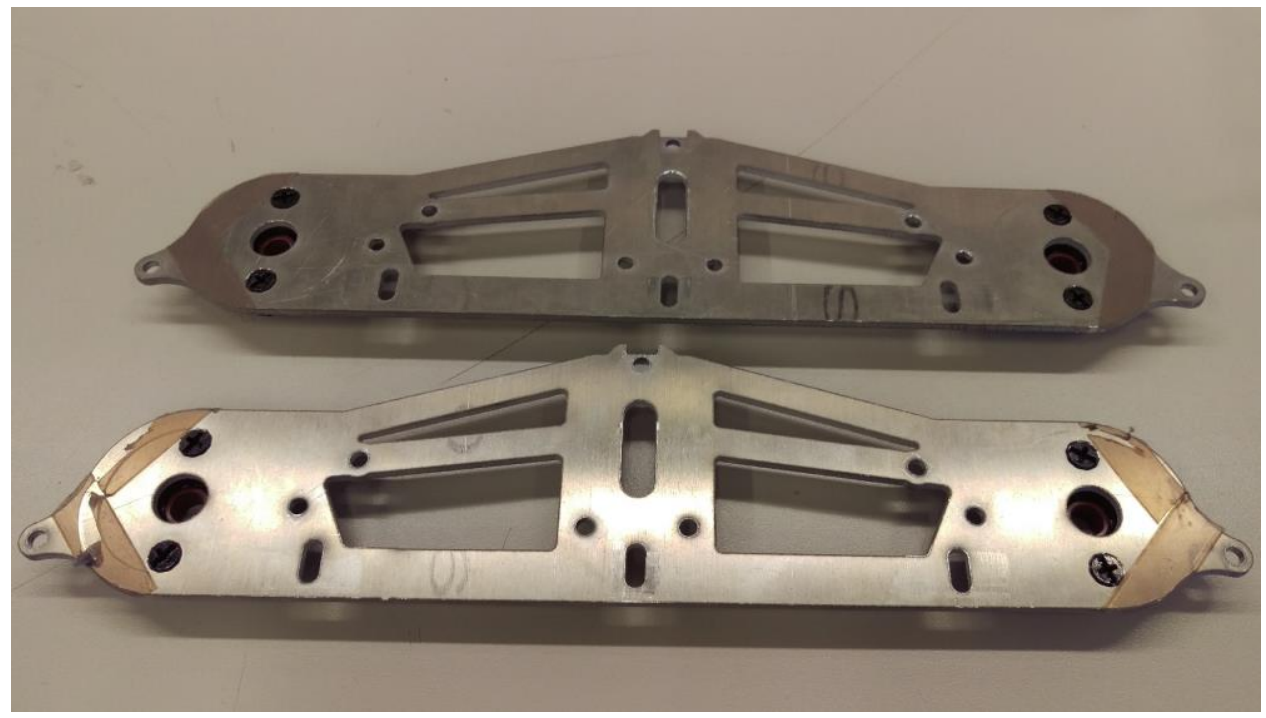

Figure 65: Damaged PTFE tape due to loose drive pulley set screws

Following the modifications explained above, the manifold pressure tests and pull-off tests were repeated, yielding the results recorded in the two tables below. Again, pressures were repeatable and consistent during multiple pull-off and replace cycles. 
Table 28: Final manifold section pressure testing results

\begin{tabular}{lllllllll}
\hline $\begin{array}{l}\text { Manifold } \\
\text { Section }\end{array}$ & 1 & 2 & 3 & 4 & 5 & 6 & 7 & 8 \\
\hline $\begin{array}{l}\text { Pressure [inHg] } \\
\text { Pressure [psi] }\end{array}$ & 25 & 24.5 & 25 & 24.5 & 25 & 25.5 & 25 & 25 \\
\hline
\end{tabular}

Table 29: Final pull-off force testing results

\begin{tabular}{ll}
\hline Property & Value \\
\hline Weight [lbf] & 7.3 \\
Peak Pull-Off Force [lbf] & 31.5 \\
Total Vacuum Force [lbf] & 24.2 \\
Vacuum Force, Calculated from Pressures in Table 28 [lbf] & 32.9 \\
Difference vs Calculation & $-26 \%$ \\
Design Criteria Vacuum Force from Table 2 [lbf] & 32.5 \\
Difference vs Design Criteria & $-25 \%$ \\
\hline
\end{tabular}

The conclusion drawn from the new manifold pressure testing results is that the manifold is sealing very well against the tread following the modifications. All manifold sections are pulling essentially equivalent vacuum. Some small leaks are present somewhere in the pneumatic system, losing approximately $1 \mathrm{psi}$ from the vacuum pressure supplied by the venturis, but such a small drop is acceptable.

The pull-off test results have also improved dramatically from the preliminary test. An increase in pull-off force of approximately $13 \mathrm{lbf}$ was realized by adjusting the manifolds and stiffening the suspension. Again, the discrepancy from the calculated values can be explained by small leaks accumulating as force is being applied to lift the vehicle. As pressures in the manifolds drop slightly due to the relatively minor leaks, the expected pull-off force for the vehicle drops as well. The ultimate force is recorded at some instant when leaks are created that are large enough to cause a critical seal failure. The important conclusion is that the geometrically calculated pressure in the manifolds matches the desired vacuum force dictated by the design criteria. 


\subsection{Vertical Maneuvering Testing on Multiple Surfaces}

The practical objective of the prototype vehicle is to demonstrate maneuverability on vertical surfaces, providing proof-of-concept for the unique locomotion/adhesion system. During the course of testing, the prototype showed capability for a range of surfaces, including: glass, painted concrete, painted plywood, painted sheet metal, and composite building siding. In the majority of tests and images below, a sophisticated, composite-reinforced elastic safety rig was employed to gently arrest any falls without damage to the vehicle. Brief descriptions of each surface set is included below.

\subsubsection{Glass Surface Testing}

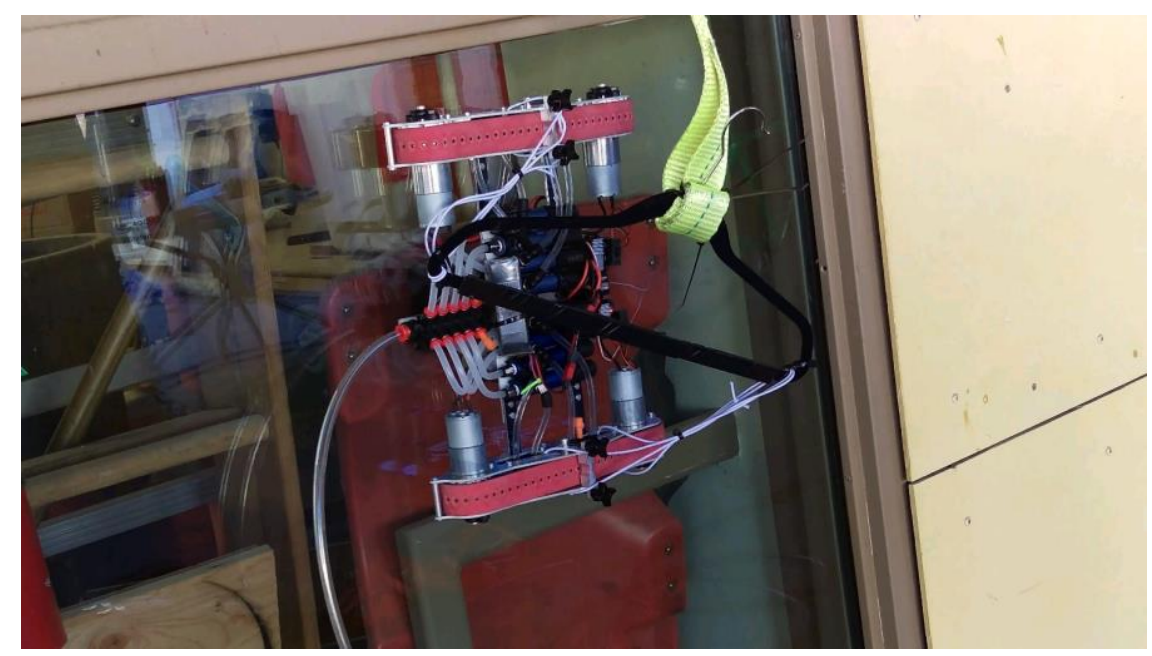

Figure 66: Video frame, horizontal traverse on glass

Glass surfaces were the first testing surface attempted, due to the high friction coefficient when paired with the Linatex tread backing. Additionally, the very smooth surface provided the best chance to seal the tread-wall interface, allowing quantitative measurements of the seal quality elsewhere in the system, as described in the previous sections. 


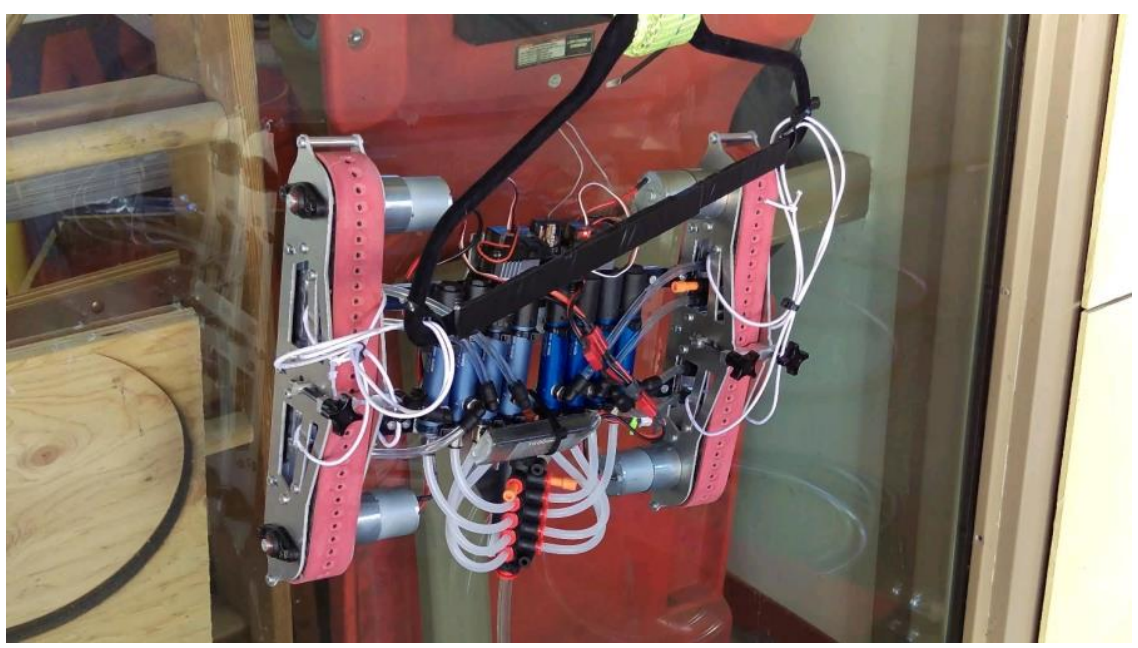

Figure 67: Video frame, vertical climb on glass

Vehicle mobility on vertical glass surfaces was achieved easily following installation of the EPDM manifold seals and Teflon taped belt channel. Static adhesion was very strong, and during maneuvering in a straight line in any vehicle orientation, the vehicle reliably traversed the surface. The adhesion was so strong that if one treadpod was detached from the wall, the remaining treadpod could carry the cantilevered weight of the entire vehicle and vertically climb or descend the wall. Skid steering and turning of the vehicle caused problems, however, often and seemingly randomly causing the vehicle to lose seal on one or both treadpods. Replacement of the spring steel suspension flanges with aluminum did not appear to improve this problem.

After consideration, the most believable theory regarding the difficulty maneuvering on glass is that the static friction and grip between the treads and the wall is high enough that the kinetic sliding motion required for skid steering causes the treadpods to catch, skip, and jump on the high friction surface, breaking the pneumatic seal on one or both sliding treadpods. A reduction in tread friction coefficient or a reduction in vacuum force may be able to decrease this friction to the point that sliding skid steering on glass is possible. 


\subsubsection{Painted Concrete and Painted Plywood}

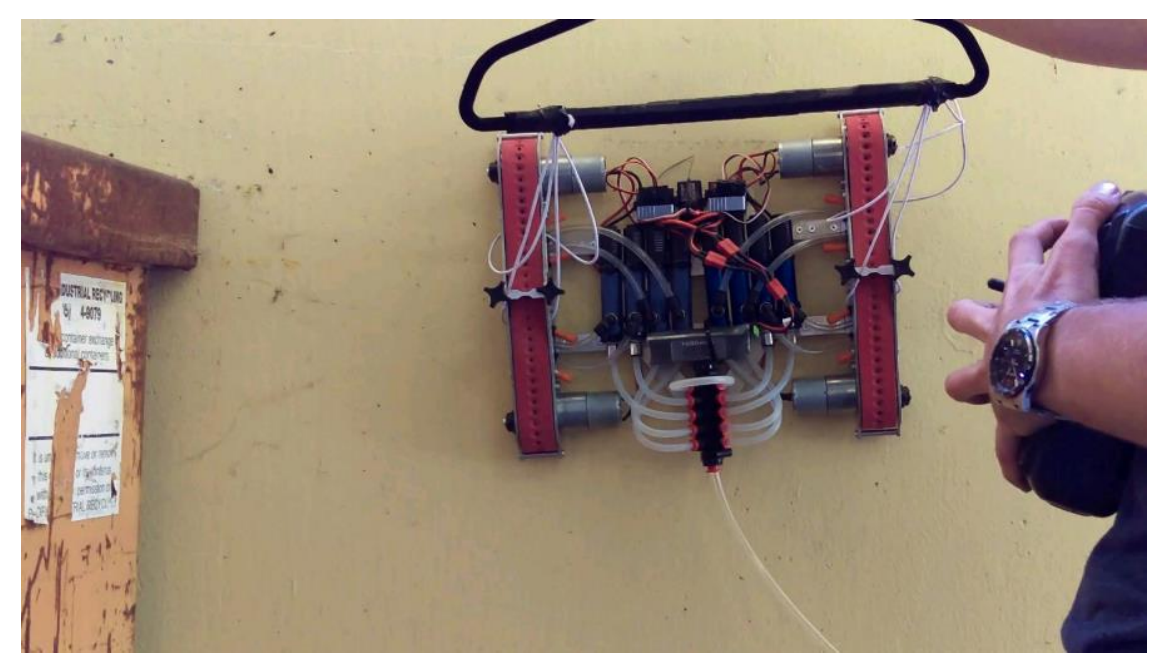

Figure 68: Video frame, vertical climb on concrete

Two dirty, relatively rough surfaces were tested: an external, painted concrete retaining wall and the plywood facing of a roll-up door. Both surfaces presented potential difficulties for the vehicle, including chipping paint, butted plywood seams, concrete surface roughness, and particulates on the surface. Neither surface was cleaned before the vehicle was applied.

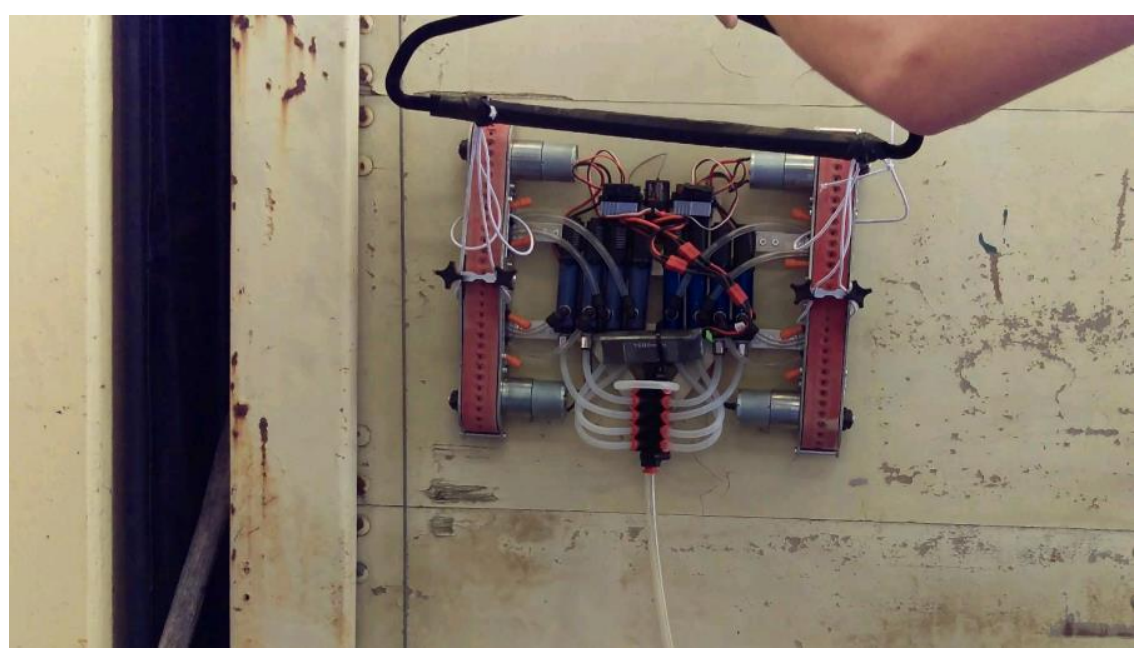

Figure 69: Video frame, vertical climb on plywood 
In practice, however, both surfaces provided sufficient adhesion. Tests on each surface consisted of vertical travel up and down the wall as well as some limited maneuverability trials. Skid steering on these surfaces proved far more reliable than on a glass surface. These trials provide an excellent proof-of-concept demonstrating the multi-surface capability of this combined locomotion and adhesion system.

\subsubsection{Composite Siding}

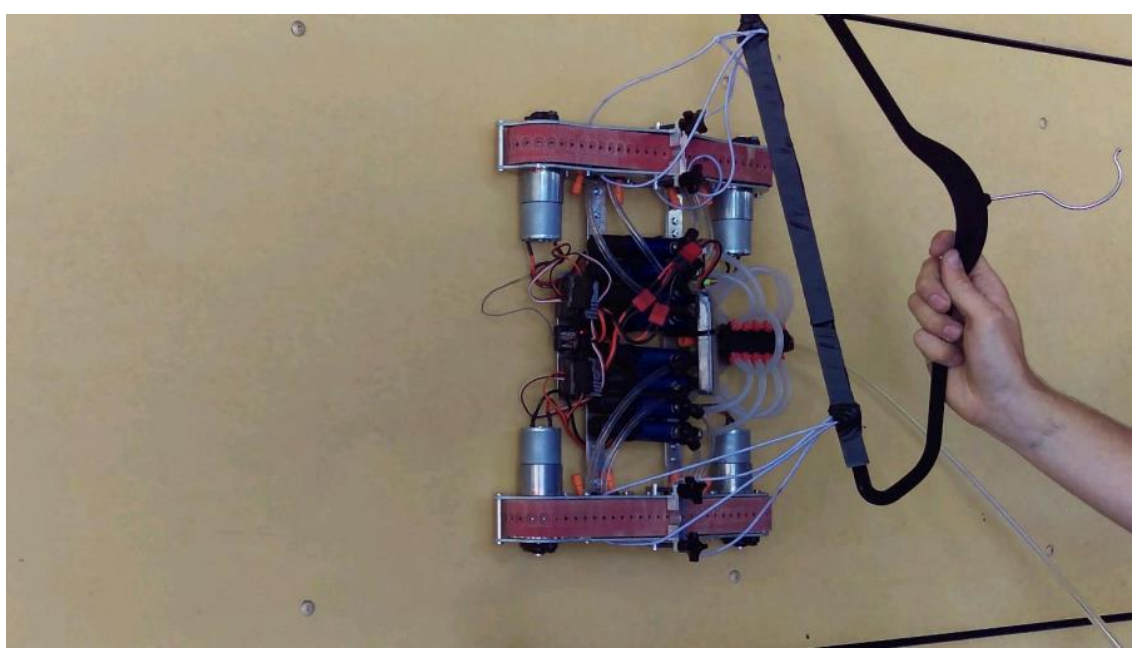

Figure 70: Video frame, horizontal traverse on composite siding

A relatively smooth surface, the composite building siding of the Cal Poly machine shops provided an intermediate surface quality between the painted surfaces above and a plate glass window. As expected, vehicle adhesion and mobility in straight paths was excellent. Skid steering was again more reliable than the glass surface, but still provided relatively frequent seal failures during sharp turning maneuvers. 


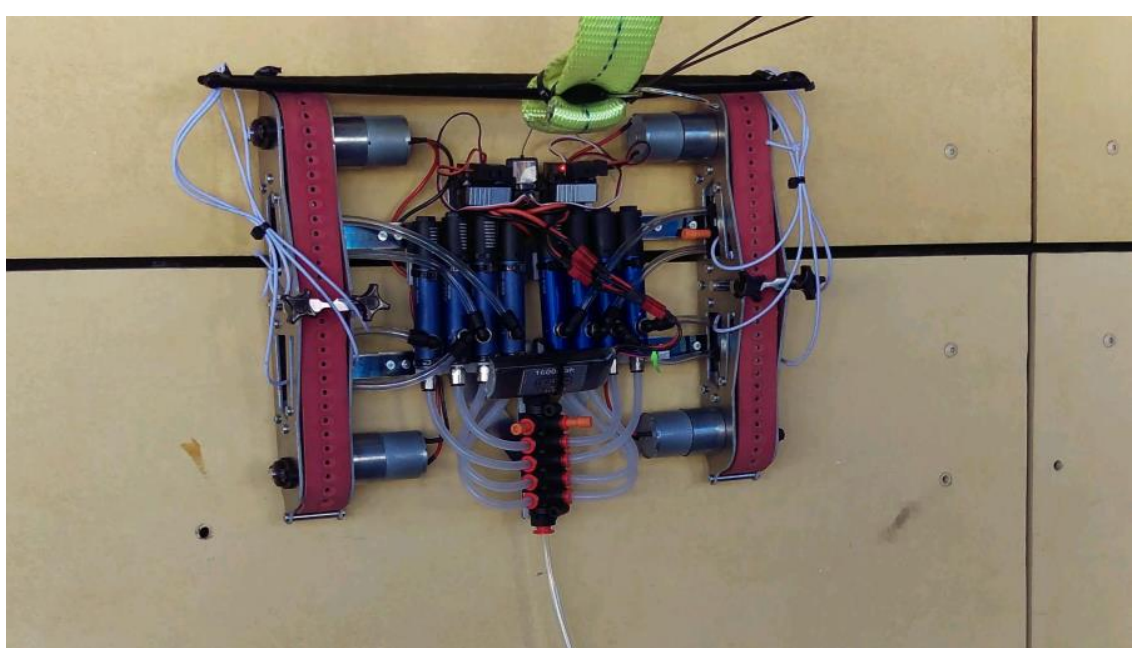

Figure 71: Video frame, gap traverse on composite siding

In addition to standard maneuverability testing, the gaps between composite panels provided an informal ability to test the rough surface and vacuum section redundancy capabilities of the vehicle. As shown in the screenshot Figure 71, the prototype was able to climb vertically from frame bottom and straddle the gap until approximately midway along the treadpod before falling. The upper composite panel is slightly recessed from the lower panel, likely reducing the vacuum force contribution of the upper manifolds to near zero. Additionally, any manifolds open the central gap will be reduced to atmospheric pressures as well. The vehicle's performance despite these difficulties proves that a reliable, redundant climbing vehicle solution may be possible with the design elements in this prototype.

\subsubsection{Painted Metal}

The most successful surface attempted during the preliminary maneuverability trials proved to be a dusty, painted, metal-faced door of a storage container. Using this surface, the vehicle was able to reliably maneuver in every direction, including skid steering. Video was recorded demonstrating the prototype, smoothly and without incident, completing a square shaped pattern on the surface. The vehicle demonstrated the ability to turn in sweeping turns 
(both treads running in the same direction), single tread turns (one tread stopped), and full pivoting turns (treads running in opposite directions).

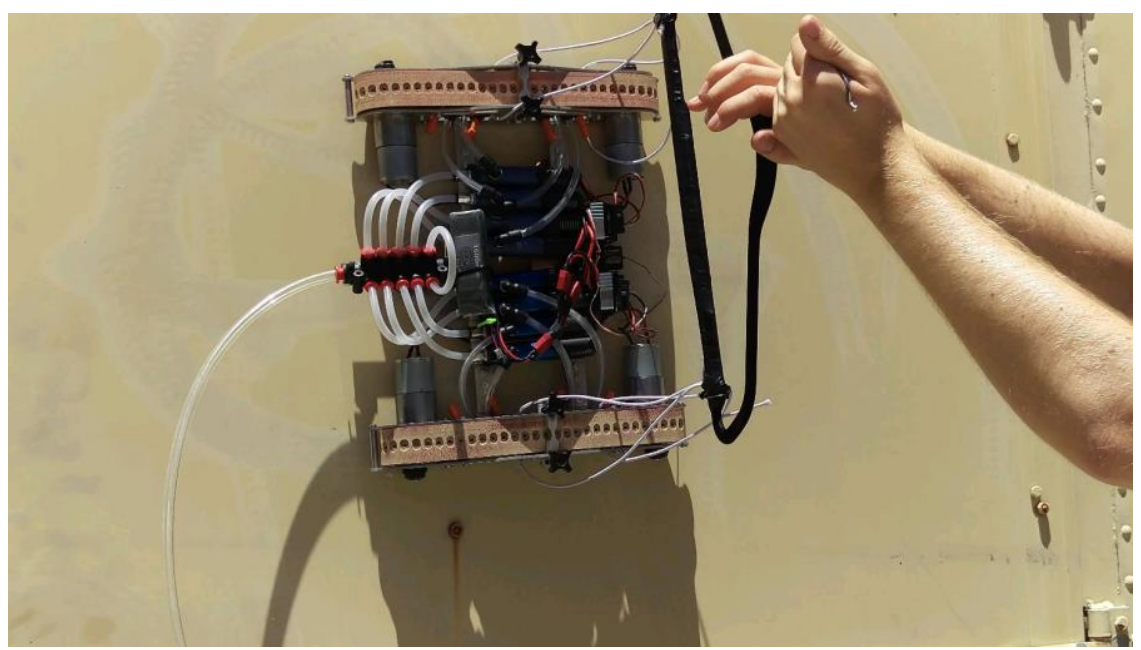

Figure 72: Video frame, final horizontal traverse in square route

The skipping and jumping observed during maneuvering on glass was entirely absent on the surface shown in Figure 72, allowing for smooth and flawless maneuvering. A combination of the dusty layer over the surface as well as a lower friction substrate below the dust likely contributed to the ideal balance of grip and slip required for such a vehicle to maneuver smoothly. 


\section{CONCLUSIONS AND RECOMMENDATIONS}

Following the success of the maneuvering trials explained above, the proof-of-concept burden on the prototype vehicle has been lifted, and clear demonstrations of a unique, promising design for a climbing vehicle has been provided. Continued testing on the current prototype as well as educated design refinement is necessary and desirable to advance this process to a more applicable, usable stage. The following paragraphs will examine each major system and summarize the performance achieved and the recommendations for future improvements.

\subsection{Subsystem Conclusions and Improvements}

\subsubsection{Tread Perforation Design}

The method of using counter-bored slots and small diameter through-holes to transmit vacuum from the vehicle manifolds to the wall surface proved a mass- and space-efficient design, which performed well. I believe that they are the clearly superior choice over through-drilled perforations and would not change the design decision made in this project.

\subsubsection{Tread Base Material}

The base material of the timing belts used, neoprene $240 \mathrm{~L}$ with fiberglass reinforcement, caused no structural or durability issued during any phase of the testing. The sole issue with the chosen base material proved to be due to the fabric layer applied to all standard neoprene belt teeth to provide smooth and quiet running. This fabric layer remained in the sections of the belt between the teeth even as the teeth themselves were removed, resulting in the rough vacuum interface surface that necessitated the troubleshooting described in the previous sections. 


\subsubsection{Tread Backing Material}

As a high-friction, wear resistant material, the Linatex performed exactly as desired. Provided a good seal path between the manifold and the wall was achieved, holding friction on even dusty or contaminated surfaces was rarely a problem during vehicle testing. However, the Linatex may have provided too much friction, resulting in the difficulty with skid steering outlined in the material testing briefs above.

\subsubsection{Vacuum Manifold and Manifold Seal}

The aluminum vacuum manifolds and the related fittings, fasteners, and attachments caused no problems during testing. The slotted adjustment fasteners allowed the manifolds to be moved and set to the correct position, exactly as designed. The mass and height saved by the twostage NPT tapping process proved to be worth the slight inconvenience of creating a new tap. The spacing of the pneumatic fittings allowed both double-inlet connections to be installed for troubleshooting without any modifications.

The vacuum manifold seal, however, proved to be the focus of the majority of the prototype's troubleshooting effort. In retrospect, the initial design, a PTFE interface with the treads, had very little chance of achieving a reliable, robust seal, even if the treads had been received from F.N. Sheppard with the as-promised smooth running surface. The rapid, highdensity series of manifold iterations provided a simple, drop-in replacement for the PTFE that allowed for the robust seal required. The EPDM foam is likely not the ideal solution, but during testing it has shown no observable signs of serious deterioration. 


\subsubsection{Venturi Pumps and Pressure Manifold}

Both off-the-shelf components, the Vaccon venturi pumps and the SMC Pneumatics pressure manifold performed without issue during testing. It was discovered after testing that the supply air in the Cal Poly machine shops is often below the recommended shop air pressure, often as low as 55-60 psi. Despite this lack of pressure, the venturi pumps provided sufficient vacuum pressure to prove function of the prototype vehicle. With full pressure, vacuum pressure and evacuation rate created by the venturis would likely increase to closer to the published pressure, improving various performance characteristics of the vehicle. The pressure manifold operated nominally, without any noticeable leaks from any of its 12 push-to-connect fittings.

\subsubsection{Motors}

The four Pololu gearmotors used on the prototype worked flawlessly. It is possible that smaller or lighter motors could be found that would perform as well or better than the Pololu motors, but their capability served the prototype vehicle well. The extremely high available torque allowed for experimentation with manifold seal materials without concern for stalling the vehicle. The high reduction allowed for reliably controllable soft starts, giving the best chance to ease the vehicle into motion without any abrupt movements. The ease of control provided by brushed, DC motors (as compared to servos or stepper motors) allowed the use of a simple hobbyist electronics. The low current draw and flexible voltage requirements allowed the vehicle to be powered by an onboard $7.4 \mathrm{~V}$ lithium polymer battery instead of an off-vehicle power supply. Finally, the motor geometry and shaft dimensions facilitated easy integration with the frame and drive system. 


\subsubsection{Pulleys and Tensioner}

Both the drive and tensioner pulleys, machined from pulley stock, were cost-, mass-, and time-efficient methods to obtain the required functions. Beyond the addition of thread locker on the motor shaft set screws, no issues with either type of pulley surfaced during testing.

The tensioner system itself worked as designed. The concern of skewing the tensioner axle with mismatched lengths of the two thumbscrews did not materialize, as it was simple to ensure the axle stayed level with the barest care when tensioning. However, the tensioner could have been designed with slightly more travel, as during testing the travel was usually at the max allowable value. No issues with lack of tension were observed, but any optimization of belt tension with vehicle performance would benefit from an additional range of achievable tension.

\subsubsection{Suspension}

The attempted suspension system on the prototype vehicle was not ultimately necessary for the vehicle's success in maneuvering on several surfaces, and the additional, unwanted flexibility introduced by the spring steel flexures may have caused seal problems in the pneumatic system. The suspension was designed to be adjusted during testing, and the adjustment performed allowed the vehicle to function properly using just the inherent stiffness of the central frame. More thought, however, should be put into a more intricate suspension system for future iterations.

\subsubsection{Frame and Structural Components}

In the effort of streamlining manufacturing, the major frame components were designed as $2 \mathrm{D}$ profiles for cutting on a water jet. Both the treadpod frames and the central frame performed as designed without major flaws. Several structural issues caused by insufficient 
review in the fast-paced design and manufacturing schedule are present, though they did not occur during testing. Several fastener holes, most notably the tensioner bracket screw, suffer from lacking edge distance from the side of the part, going against general good engineering practices. Additionally, the attachment points of the suspension brackets on the treadpod frame are positioned in the middle of a thin, unsupported frame span. They could, in a fall or impact, cause serious bending of the frame, requiring replacement.

The secondary structural components performed well, though it is unclear how essential each bracket, support, or standoff was to the performance of the vehicle. It is possible that some weight could be saved by cutting back the number of supports, though it was not deemed wise to do before a functioning prototype was created. The cable ties and cable tie mounting strategy, however, was sturdy, lightweight, and easy. I have no reservations to recommend this attachment method for future vehicles.

\subsubsection{Control Systems}

The use of hobby-grade control electronics provided simple, hassle-free control of the motor systems. Once initial setup was completed, no additional work was necessary to keep the system functioning as desired, and no problems were discovered. More sophisticated control systems, possibly incorporating small pressure transducers for monitoring adhesion, encoders to ensure synchronized motors, and signal mixing to allow for control from a more conventional control stick configuration would certainly take this system to a higher level.

\subsection{Recommendations for Future Work}

The prototype explained in the preceding pages has proven the capability of a vehicle using a combined adhesion/locomotion system to climb multiple surfaces and maneuver vertically. Direct modifications to the designed vehicle may improve its capabilities, and I believe 
that the general structural framework of the vehicle is a good platform for experimentation. These modifications should focus on designing a superior, more robust seal between the drive treads and the manifolds. As shown in this design, a sliding seal interface functions best with at least one compliant interface to account for vehicle movement, surface roughness, and other uncontrollable features. This seal could be made more reliable by the creation of a U-shaped sealing channel in which the manifold can ride, allowing for sealing to occur on vertical walls on either side of the manifold as well as the flat base. Fully custom drive treads may be required to achieve a welldesigned, effective solution.

As designed, however, the vehicle has several limitations that prevent it from being applied to many possible functions in industry. The next iterations of this vehicle should, in my opinion, must focus on several aspects: capability to travel on geometrically interesting surfaces, capability to transition from horizontal from vertical (and vise-versa), and smarter multi-surface capabilities.

The domain of the vehicle in this report is solely flat, two dimensional planar surfaces. Due to the flat, rigid vacuum manifold design as well as the current state of the system formerly known as suspension, any curvature of the climbing surface will result in broken vacuum seals between the treads or manifolds and the surface. As one of the more promising potential applications of this technology involves inspection of large, cylindrical or spherical pressure vessels, this capability cannot be overstated. Clearly, a more advanced suspension system will be a requirement to maintain vacuum contact and seal against changing surfaces. It is likely that this advancement will require moving away from the timing belt drive, and that is not unwarranted. Modified off-the-shelf timing belts have many limitations, some of which were observed in this project. A new, custom perforated drive system will be essential.

As a semi-autonomous vehicle, this robot will need to be able to transition from ground to wall and back again without direct human interference. A possible method for this involves articulating or pivoting trains of manifold sections which can raise themselves up onto the desired 
wall, and then pull the remainder of the vehicle onto the wall behind them. There are certainly many more options for this capability.

One of the surprising shortfalls of this prototype vehicle was the problems caused by excessive grip on several surfaces. In an attempt to ensure sufficient frictional capability, the vehicle hamstrung itself by preventing the smooth sliding motions required for maneuvering. Optimization of tread friction for various surfaces is one approach that could help to solve this problem. Possibly more helpful, however, would be the implementation of a control system that can reduce or increase the manifold vacuum pressure in order to maintain sufficient but not excessive grip on the climbing surfaces.

Regardless of the direction that this project follows, the promising technology shown in this report will hopefully, in the future, be advanced to the point where it can carve out a differentiated and useful niche in industrial, commercial, or private use. 


\section{BIBLIOGRAPHY}

[1] D. Longo and G. Muscato, "The Alicia Climbing Robot," IEEE Robotics and Automation Magazine, pp. 42-50, 2006.

[2] E. Ackerman, "Robot Uses Supersonic Jets of Air to Stick to Almost Anything," IEEE Spectrum, 24 MAy 2011. [Online]. Available: http://spectrum.ieee.org/automaton/robotics/industrial-robots. [Accessed June 2017].

[3] P. Beardsley, P. D. R. Siegwart, M. Arigoni and M. Bischoff, "VertiGo - A Wall-Climbing Robot including Ground-Wall Transition," Disney Research, 29 December 2015. [Online]. Available: https://www.disneyresearch.com/publication/vertigo/. [Accessed June 2017].

[4] T. Lombardo, "Climbing Robotic Wind Turbine Inspector," 28 April 2013. [Online]. Available: http://www.engineering.com/ElectronicsDesign/ElectronicsDesignArticles/ArticleI D/5652/Climbing-Robotic-Wind-Turbine-Inspector.aspx. [Accessed June 2017].

[5] L. Kelley, S. Ostovari, A. B. Burmeister and K. A. Talke, "Design and experimental validation of a simple controller for a multi-segment magnetic crawler robot," Space and Naval Warfare Systems Center Pacific, San Diego, 2015.

[6] G. S. Virk, F. Rochat, P. Schoeneich and F. Mondada, "TRIPILLAR: Miniature magnetic caterpillar climbing robot with plane transition ability," ResearchGate, 2009.

[7] Y. Yoshida and S. Ma, "Design of a Wall-Climbing Robot with Passive Suction Cups," IEEE International Conference on Robotics and Biomimetics, 2010. 
[8] H. Kim, D. Kim and H. Yang, "A wall climbing robot with vacuum caterpillar wheel system operated by mechanical valve," Proceedings of the 9th International Conference on Climbing and Walking Robots, pp. 28-33, 2006.

[9] International Climbing Machines, "The Climber," [Online]. Available: http://www.icm.cc/climber.php.

[10] D. Graham-Rowe, "Building a Better Wall Climber," MIT Technology Review, 29 May 2008. [Online]. Available: https://www.technologyreview.com/s/410195/buildinga-better-wall-climber/. [Accessed June 2017].

[11] M. Spenko, G. HAynes, J. Saunders and M. Cutkosky, "Biologically Insipred Climbing with a Hexapedal Robot," Kod*lab.

[12] A. Saunders, D. Goldman, R. Full and M. Buehler, "The RiSE Climbing Robot: Body and Leg Design," Department of Integrative Biology, University of California, Berkeley.

[13] T. L. Lam and Y. Xu, "Tree Climbing Robot - Design, Kinematics, and Motion Planning," Springer Tracts in Advanced Robotics, vol. 78, 2012.

[14] J. Liu, Z. Tong and J. Fu, "A gecko inspired fluid driven climbing robot," in IEEE International Conference on Robotics, 2011.

[15] J. Stefani, "The Design and Testing of a Novel Combined Adhesion and Locomotion Method for Wall Climbing Vehicles," California Polytechnic State University, San Luis Obispo, 2016.

[16] Fengyu, Xu, S. Jingjin and J. GuoPing, "Kinematic and Dynamic Analysis of a Cableclimbing Robot," International Journal of Advanced Robotic Systems, 2015.

[17] Gates Mextrol, Inc., "Belt Backings - Specifications," Gates Mectrol, Inc., Salem, 2008. 
[18] Brecoflex Co., L.L.C, "Backing Materials," 10 April 2017. [Online]. Available:

http://www.brecoflex.com/products/backings/backing-materials/.

[19] Weir, "Linatex," 15 April 2017. [Online]. Available:

https://www.global.weir/brands/linatex/.

[20] S. Biswas, "Friction and wear of PTFE - a review," Wear, pp. 193-211, 1992.

[21] F.N Sheppard \& Co., "Belt Design Catalog," F.N. Sheppard \& Co., Erlanger, 2000.

[22] Gates Mectrol, Inc., "Belt Sizing Guide," Gates Mectrol, Inc, Salem, 2006.

[23] R. G. Budynas and K. J. Nisbett, Shigley's Mechanical Engineering Design, 9 ed., Boston: The McGraw-Hill Companies, Inc., 2011.

[24] T. T. Baber, "Torsion in Structural Design," 5 May 2017. [Online]. Available: http://people.virginia.edu/ ttb/torsion.pdf. 


\section{APPENDICES}

\section{A. DESIGN CALCULATIONS AND ANALYSIS}

\section{A.1 Vacuum Area Required, Global}

The most basic and most necessary calculation performed during prototype design is the global static vehicle FBD. The following short section overviews the problem. The free body diagram is displayed below:

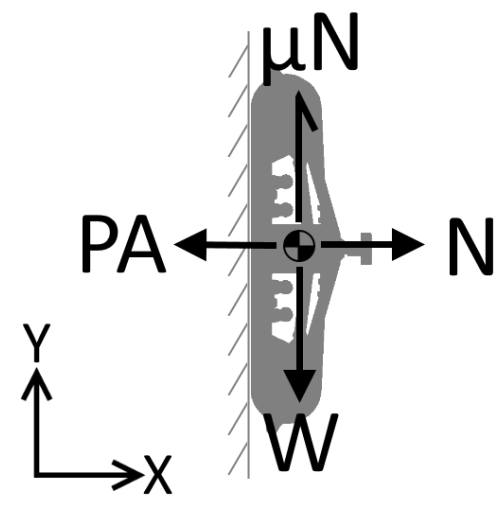

Figure 73: Global free body diagram

Table 30: Global FBD variables

\begin{tabular}{lll}
\hline Variable & Name & Value \\
\hline $\mathrm{W}$ & Design Weight [lbf] & 15 \\
$\mathrm{P}$ & Vacuum Pressure [psi] $^{2}$ & 13 \\
$\mathrm{~A}$ & Wall Area Exposed [in. $^{2}$ ] & Calculated \\
$\mu$ & Static Coefficient, Tread to Wall & 0.75 \\
$\mathrm{~N}$ & Normal Force [lbf] & Calculated \\
\hline
\end{tabular}

The objective of this calculation is to determine the required vacuum area (A) exposed to the wall in order to ensure the vehicle has sufficient friction $(\mu \mathrm{N})$ to counteract the weight of the vehicle. The first step is to take the sum of forces along the $\mathrm{X}$ axis, which simply yields:

$$
\begin{gathered}
N-P A=0 \\
N=P A
\end{gathered}
$$

Next, sum forces in the $\mathrm{Y}$ direction, and substitute the $\mathrm{X}$ direction result for $\mathrm{N}$. 


$$
\begin{gathered}
\mu N-W=0 \\
\mu(P A)-W=0 \\
A=\frac{W}{\mu P}
\end{gathered}
$$

Insert the values from the variable table and solve for A.

$$
\begin{gathered}
A=\frac{15 \mathrm{lbf}}{0.75(13 \mathrm{psi})} \\
A=1.54 \text { in. }^{2}
\end{gathered}
$$

Thus, the vacuum area required to counteract the vehicle weight is $1.54 \mathrm{in}^{2}$. This will result in a total vacuum force of $20 \mathrm{lbf}$ in order to hold up the $15 \mathrm{lbf}$ weight vehicle.

\section{A.2 Additional Area for Peel-Off}

The above global calculation does not take into account the moment caused by the CG's height off of the wall. This moment causes a "peel-off" moment that tries to pull the top of the vehicle backwards off of the wall. In order to obtain a simplified accounting of the effective "additional vacuum area" required. Several assumptions are made in this calculation, and they are laid out below.

The primary assumption is that, in order to simplify this problem to the level of a basic hand calculation, the vehicle is assumed to be supported by a pivot at one quarter tread height, and a restoring vacuum force is applied at three quarters tread height. This approximates peel-off by concentrating the distributed force applied by the upper half of the vacuum manifold to a single force at its geometric center. The adhesive vacuum force of the lower half of the treads are ignored, and the pivot at one-quarter tread height approximates the point that the vehicle would rotate about as the vehicle peels off of the climbing surface. 
The objective of this calculation is to determine the amount of additional vacuum area required at each side (upper and lower) of the vehicle to offset the peel-off moment. The FBD and calculation steps are included below.

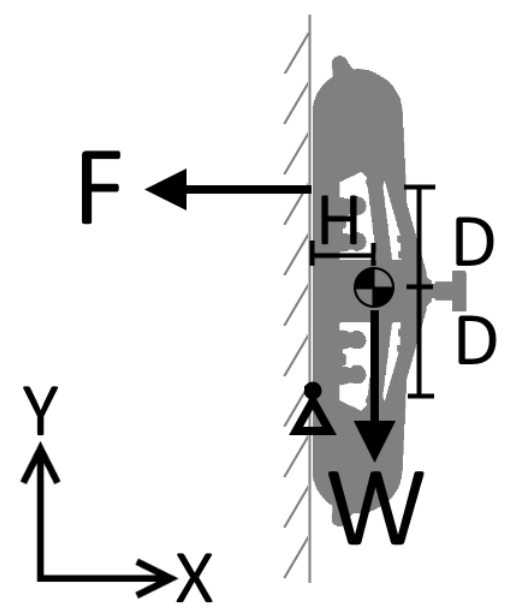

Figure 74: Peel-off force free body diagram

Table 31: Peel-off force calculation variables

\begin{tabular}{lll}
\hline Variable & Name & Value \\
\hline F & Additional Vacuum Force & Calculated \\
D & CG to Upper/Lower Manifold Center [in.] & 3 \\
$\mathrm{H}$ & CG Height [in.] & 3 \\
$\mathrm{~W}$ & Design Weight [lbf] & 15 \\
$\mathrm{P}$ & Vacuum Pressure [psi] & 13 \\
$\mathrm{~A}$ & Additional Vacuum Area [in. $\left.{ }^{2}\right]$ & Calculated \\
\hline
\end{tabular}

Take a moment sum about the pivot point and set the result equal to zero (static conditions). Solve for F.

$$
\begin{gathered}
F(2 D)-W(H)=0 \\
F=\frac{W H}{2 D}
\end{gathered}
$$

The additional vacuum force can be further simplified to the product of pressure and additional vacuum area.

$$
P A=\frac{W H}{2 D}
$$




$$
A=\frac{W H}{2 D P}
$$

Insert values into the equation for the additional vacuum area.

$$
\begin{gathered}
A=\frac{(15 \mathrm{lbf})(3 \mathrm{in} .)}{2(3 \mathrm{in} .)(13 \mathrm{psi})} \\
A=0.576 \text { in. }^{2}
\end{gathered}
$$

Thus, the top half of the treadpods must have approximately .576 in. ${ }^{2}$ additional area to counteract the peel-off force. Applying this logic further, that means that the bottom half of the treads must also have the same additional area added to them. This results in a total additional area of 1.15 in. $^{2}$.

This additional area must be added to the basic, global vacuum area calculated previously. Thus, the total vacuum area required for the vehicle is:

$$
\begin{gathered}
A_{\mathrm{TOTAL}}=A_{\mathrm{GLOBAL}}+2 A_{\mathrm{PEEL}} \\
A_{\mathrm{TOTAL}}=1.54 \mathrm{in}^{2}+2\left(.576 \mathrm{in}^{2}\right) \\
A_{\mathrm{TOTAL}}=2.50 \mathrm{in}^{2}
\end{gathered}
$$

\section{A.3 Motor Torque}

In order to provide power to the treads, the motors must output enough torque to lift the entire weight of the vehicle as well as overcome the friction between the treads and the vacuum manifold. The following calculation was performed on a single treadpod, as each treadpod is independent of the other. Thus, weight is halved. Additionally, the vacuum area of manifold exposed to the tread is assumed a $6 \mathrm{in}$. long, $0.25 \mathrm{in}$. wide slot, or $1.5 \mathrm{in} .^{2}$ per tread. 


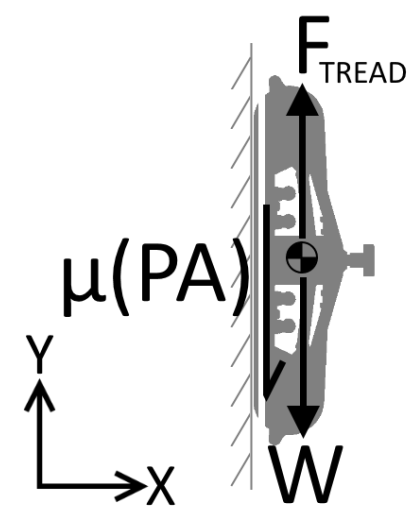

Figure 75: Motor torque free body diagram

Table 32: Motor torque calculation variables

\begin{tabular}{lll}
\hline Variable & Name & Value \\
\hline F $_{\text {TREAD }}$ & Tread Pulling Force [lbf] & Calculated \\
$\mu$ & Static Coefficient, Tread to Manifold & 0.4 \\
$\mathrm{P}$ & Vacuum Pressure [psi] & 13 \\
$\mathrm{~A}$ & Vacuum Manifold Area on Treads [in. ${ }^{2}$ ] & 1.5 \\
$\mathrm{~W}$ & Vehicle Weight, Half [lbf] & 7.5 \\
$\mathrm{R}$ & Drive Pulley Radius [in.] & 1 \\
$\mathrm{~T}$ & Motor Torque, Total [in. $\left.{ }^{*} \mathrm{lbf}\right]$ & Calculated \\
\hline
\end{tabular}

Some simplification has already been performed in this analysis. Namely, the friction interaction between the manifold and the tread in the $\mathrm{X}$ direction has been solved to prove that the normal force of the treads on the manifold is equal to the vacuum pressure in the manifold multiplied by the area exposed to that vacuum, or:

$$
N=P A
$$

Thus, the normal force term in the vertical friction force has already been replaced.

Continue by summing forces along the $\mathrm{Y}$ axis.

$$
\begin{gathered}
F_{\mathrm{TREAD}}-W-\mu(P A)=0 \\
F_{\mathrm{TREAD}}=W+\mu(P A)
\end{gathered}
$$

Now we can calculate the force required to pull the tread to allow the vehicle to climb. Torque can be extracted from this term to obtain a more useful criterion for the motor selection.

$$
T=F_{\mathrm{TREAD}} * R
$$


Thus:

$$
T=[W+\mu(P A)] * R
$$

Insert the known values into the expression to determine torque required.

$$
\begin{gathered}
T=\left[7.5 \mathrm{lbf}+0.4(13 \mathrm{psi})\left(1.5 \mathrm{in.}^{2}\right)\right] * 1 \mathrm{in} \\
T=15.3 \mathrm{in} .{ }^{*} \mathrm{lbf}
\end{gathered}
$$

For a dual-motor setup such as this prototype, the approximate torque required for each motor is half of the total.

$$
T_{\mathrm{EACH}}=7.7 \mathrm{in.} \cdot \mathrm{lbf}
$$

\section{A.4 Vacuum Manifold Slot Length}

This section walks through sample calculations to determine the required length of belt, given a certain perforation pattern. Three styles are examined, including a single row of holes, staggered row of holes, and rounded slots.

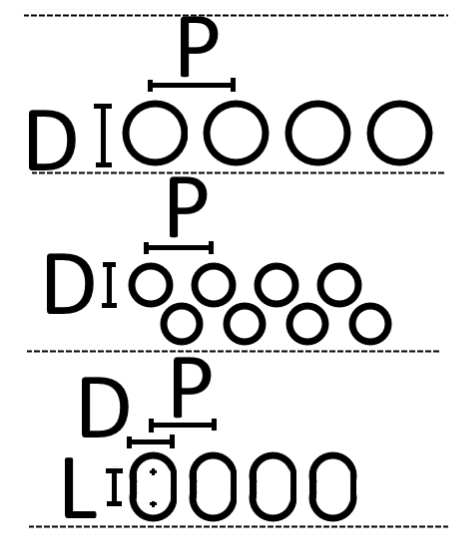

Figure 76: Tread perforation area diagram

Table 33: Tread perforation variables

\begin{tabular}{lllll}
\hline Variable & Name & Single Row & Staggered & Slots \\
\hline $\mathrm{D}$ & Diameter [in.] & 0.375 & 0.188 & 0.25 \\
$\mathrm{P}$ & Pitch [in.] & 0.469 & 0.234 & 0.375 \\
$\mathrm{~L}$ & Slot Length [in.] & -- & -- & .15 \\
$\mathrm{~A}$ & Area Req'd [in. ${ }^{2}$ ] & 1.35 & 1.35 & 1.35 \\
$\mathrm{~L}_{\mathrm{M}}$ & Manifold Length [in.] & Calc & Calc & Calc \\
\hline
\end{tabular}


The calculation for the single row of holes is straightforward. The total required vacuum area has been calculated above to be $2.69 \mathrm{in}^{2}$ for the entire vehicle. For each tread, therefore, you need half of that area, or $1.35 \mathrm{in}^{2}$. Thus, the expression for required tread length is as follows:

$$
\begin{gathered}
L_{M}=\frac{A}{\frac{\pi}{4} D^{2}} P \\
L_{M}=\frac{1.35 \text { in. }^{2}}{\frac{\pi}{4}(.375 \text { in. })^{2}}(.469 \text { in. }) \\
L_{M}=5.73 \text { in. }
\end{gathered}
$$

Therefore, 5.73 in. of tread length must be exposed to the vacuum manifolds to achieve the required vacuum area. Staggered holes are calculated in roughly the same way, except that there are two rows, so the length equation has a denominator of 2.

$$
\begin{gathered}
L_{M}=\frac{\left(\frac{A}{\pi} \frac{\pi}{4} D^{2} P\right.}{2} \\
L_{M}=\frac{\left(\frac{1.35 \text { in. }^{2}}{\frac{\pi}{4}(.188 \text { in. })^{2}}(.234 \text { in. })\right)}{2} \\
L_{M}=5.69 \text { in. }
\end{gathered}
$$

Repeating the process with the rounded slots returns the final manifold length value.

$$
\begin{gathered}
L_{M}=\frac{A}{\frac{\pi}{4} D^{2}+L D} P \\
L_{M}=\frac{1.35 \text { in. }^{2}}{\frac{\pi}{4}(.25 \text { in. })^{2}+(.15 \text { in. })(.25 \text { in. })}(.375 \text { in. }) \\
L_{M}=5.84 \text { in. }
\end{gathered}
$$

Each of these three perforation patterns, as dimensioned, therefore, have approximately the same specific vacuum area, or vacuum area per unit length of tread. Ultimately, these required 
lengths were rounded to $6.0 \mathrm{in}$. in order to simplify manufacturing and design as well as for a small amount of added safety factor.

$$
L_{M-D E S I G N}=6.00 \mathrm{in}
$$

\section{A.5 Tensioner Axle Analysis}

In order to prove that a steel tensioner axle is not necessary, some calculations were performed to show capability of the proposed .3125 in. diameter aluminum axle. A series of hand calculations were performed. First, the axle was modeled as simply supported. This assumes that the threaded rods in the ends of the axle do not resist any moment, which is a reasonable assumption. Additionally, the loading is assumed to be worst case 3-point-bending. In reality, the tensioner pulley bushings will apply the load closer to the supports reducing the bending moments significantly. Further, because F.N. Sheppard was unable to provide material properties for the fiberglass-reinforced nylon belts, the analysis becomes a worst-on-worst limit analysis.

The resulting numerical results will be the maximum force able to be applied to the axle, the deflection at that force, and the belt tension available with that max loading, assuming the tensioner is at max travel above the normal belt path.

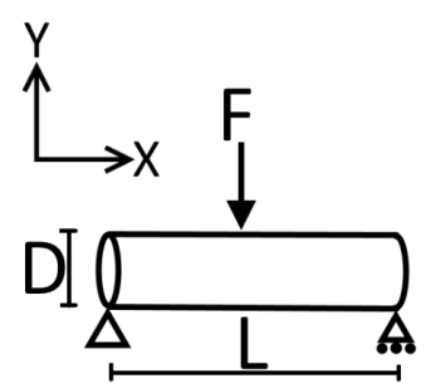

Figure 77: Tensioner axle diagram 
Table 34: Tensioner axle variables

\begin{tabular}{lll}
\hline Variable & Name & Value \\
\hline $\mathrm{D}$ & Axle Diameter [in.] & 0.625 \\
$\mathrm{~L}$ & Axle Length [in.] & 2.25 \\
$\mathrm{E}$ & Modulus of Elasticity, Aluminum [psi] & $10 \mathrm{E} 6$ \\
$\sigma$ & Yield Strength, 6061-T6 [psi] & $40 \mathrm{E} 3$ \\
$\mathrm{I}$ & Area Moment of Inertia [in. $\left.{ }^{4}\right]$ & Calculated \\
$\mathrm{M}$ & Moment at Loading [in. $\left.{ }^{*} \mathrm{bf}\right]$ & Calculated \\
$\mathrm{F}$ & Applied Central Force [lbf] & Calculated \\
$\delta$ & Max Axle Displacement [in.] & Calculated \\
$\mathrm{h}$ & Max Tensioner Travel [in.] & 0.53 \\
$\mathrm{~W}$ & Belt Span Width [in.] & 9 \\
$\mathrm{~T}$ & Max Tension [lbf] & Calculated \\
\hline
\end{tabular}

To determine the maximum possible force available, a standard materials mechanics equation can be used.

$$
\sigma=\frac{M \frac{D}{2}}{I}
$$

The moment at the center of the beam is simply defined as:

$$
M=F \frac{L}{2}
$$

The area moment of inertia of a circle is defined as:

$$
I=\frac{\pi D^{4}}{64}
$$

Therefore, substituting and solving for the allowable force, we obtain the following expressions.

$$
\begin{gathered}
\sigma=\frac{F \frac{L D}{4}}{\frac{\pi D^{4}}{64}} \\
F=\frac{4 \sigma \frac{\pi D^{4}}{64}}{L D} \\
F=\frac{4(40 E 3 \mathrm{psi}) \frac{\pi(0.313 \mathrm{in} .)^{4}}{64}}{(2.25 \mathrm{in} .)(.313 \mathrm{in} .)} \\
F=106.5 \mathrm{lbf}
\end{gathered}
$$


Thus, the max force that a .3125 in. tensioner axle can withstand before yielding is 106.5 lbf. To determine the deflection of the beam at that force, apply a beam deflection equation.

$$
\begin{gathered}
\delta=\frac{F L^{3}}{3 E I} \\
\delta=\frac{(106.5 \mathrm{lbf})(2.25 \mathrm{in} .)^{3}}{3(10 E 6 \mathrm{psi})\left(\frac{\pi(0.313 \mathrm{in} .)^{4}}{64}\right)} \\
\delta=.086 \mathrm{in} .
\end{gathered}
$$

To determine the max tension that the axle can withstand before failing, assume the max load of $106.5 \mathrm{lbf}$ is being applied to the tensioner axle by the tensioned belt. The tensioner is raised 0.53 in. above horizontal and the length of the span of the belt is 9 in. Solve the FBD shown for $\mathrm{T}$.

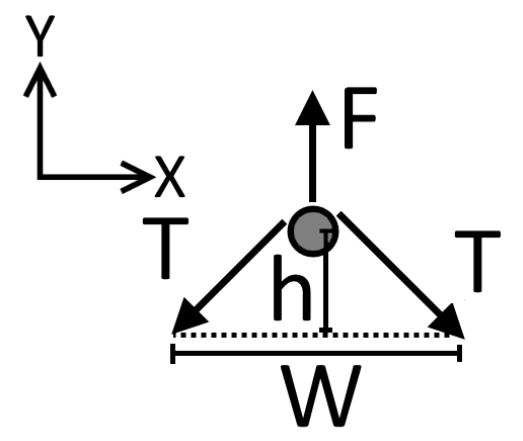

Figure 78: Belt tension diagram

Take the sum of forces in the $\mathrm{Y}$ direction. Use trigonometry to separate the components of $\mathrm{T}$ into $\mathrm{X}$ and $\mathrm{Y}$ directions.

$$
\begin{gathered}
F-2 T \sin \left(\tan ^{-1}\left(\frac{h}{W / 2}\right)\right)=0 \\
T=\frac{F}{2 \sin \left(\tan ^{-1}\left(\frac{h}{W / 2}\right)\right)} \\
T=\frac{106.5 \mathrm{lbf}}{2 \sin \left(\tan ^{-1}\left(\frac{0.53 \text { in. }}{(9 \text { in. }) / 2}\right)\right)} \\
T=482 \mathrm{lbf}
\end{gathered}
$$


This calculation shows that a belt tension of nearly $500 \mathrm{lbf}$ would be required to fail the tensioner axle. As an order of magnitude assessment, this result removes any concern of the axle failing, as the limit of tension for the project's belt is far below $482 \mathrm{lbf}$.

\section{A.6 Suspension Analysis}

Analysis of the spring steel flexure design was performed to obtain approximations of the forces required to actuate the suspension. Specifically, the motions of interest included vertical cantilever deflection and torsion. Two flexure suspension designs were analyzed: single flexure and dual, spaced flexures.

A.6.1 Single Flexure Analysis
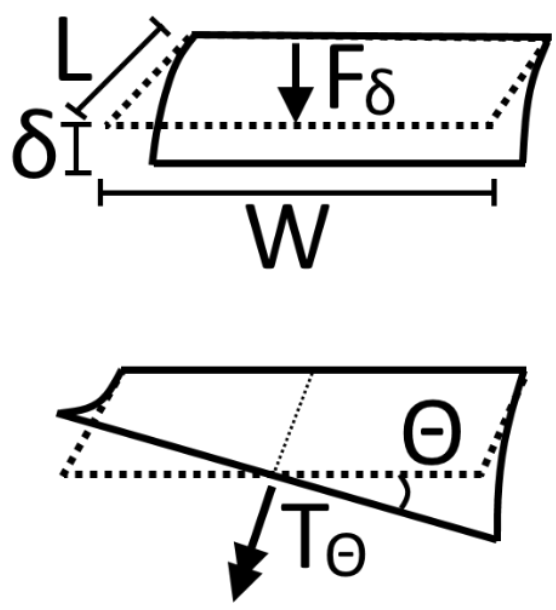

Figure 79: Single flexure diagram 
Table 35: Single flexure variables

\begin{tabular}{lll}
\hline Variable & Name & Value \\
\hline $\mathrm{T}$ & Flexure Thickness [in.] & 0.025 \\
$\mathrm{~W}$ & Flexure Width [in.] & 2 \\
$\mathrm{~L}$ & Overhung Length [in.] & 1.18 \\
$\mathrm{E}$ & Modulus of Elasticity, Steel [psi] & $30.0 \mathrm{E} 6$ \\
$\mathrm{G}$ & Shear Modulus, Steel [psi] & $11.5 \mathrm{E} 6$ \\
$\mathrm{~J}$ & Torsional Constant [in. ${ }^{4}$ ] & Calculated \\
$\mathrm{I}$ & Area Moment of Inertia [in. ${ }^{4}$ ] & Calculated \\
$\delta$ & Vertical Deflection [in.] & 0.125 \\
$\Theta$ & Twist Angle [degrees] & 3 \\
$\mathrm{~F}_{\delta}$ & Force Required [lbf] & Calculated \\
$\mathrm{T}_{\Theta}$ & Torque Required [in. $\left.{ }^{*} \mathrm{lbf}\right]$ & Calculated \\
\hline
\end{tabular}

The calculation of the vertical force required to deflect the flexure a distance $\delta$ is a simple material mechanics exercise. The equation provided in reference [23] states:

$$
\delta=\frac{F L^{3}}{3 E I}
$$

or:

$$
F=\frac{3 \delta E I}{L^{3}}
$$

Thus, the area moment of inertia, I, must be determined. For rectangular cross sections about the neutral axis, I is defined as:

$$
I=\frac{1}{12} W T^{3}
$$

Therefore, substituting back into the previous equations and plugging in know values yields the force required.

$$
\begin{gathered}
F_{\delta}=\frac{3 \delta E\left(\frac{1}{12} W T^{3}\right)}{L^{3}} \\
F_{\delta}=\frac{(.125 \mathrm{in} .)(30 E 6 \mathrm{psi})(2 \mathrm{in} .)(0.025 \mathrm{in} .)^{3}}{4(1.18 \mathrm{in} .)^{3}} \\
F_{\delta}=17.8 \mathrm{lbf}
\end{gathered}
$$


The determination of the torque required to twist the flexure requires somewhat more approximation. Rectangular beam torsion has been extensively studied [24], and for a beam of uniform cross section, the expression for angle of twist is:

$$
\Theta=\frac{T_{\Theta} L}{J G}
$$

Where $\mathrm{J}$ is the torsional constant. For thin cross section materials with the ratio of width to thickness greater than $10, \mathrm{~J}$ can be approximated as:

$$
J \approx \frac{1}{3} W T^{3}
$$

Substituting this approximation into the first equation and solving for the torque, we obtain

$$
\begin{gathered}
\Theta=\frac{T_{\Theta} L}{\left(\frac{1}{3} W T^{3}\right) G} \\
T_{\Theta}=\frac{\Theta\left(\frac{1}{3} W T^{3}\right) G}{L}
\end{gathered}
$$

Inserting numerical values returns the torque required, in in*lbf.

$$
\begin{gathered}
T_{\Theta}=\frac{(3 \text { degrees })\left(\frac{2 \pi}{360}\right)\left(\frac{1}{3}(2 \mathrm{in.})(0.025 \mathrm{in} .)^{3}\right)(11.5 E 6 \mathrm{psi})}{1.18 \mathrm{in.}} \\
T_{\Theta}=5.25 \mathrm{in.} * \mathrm{lbf}
\end{gathered}
$$


A.6.2 Double Flexure Analysis

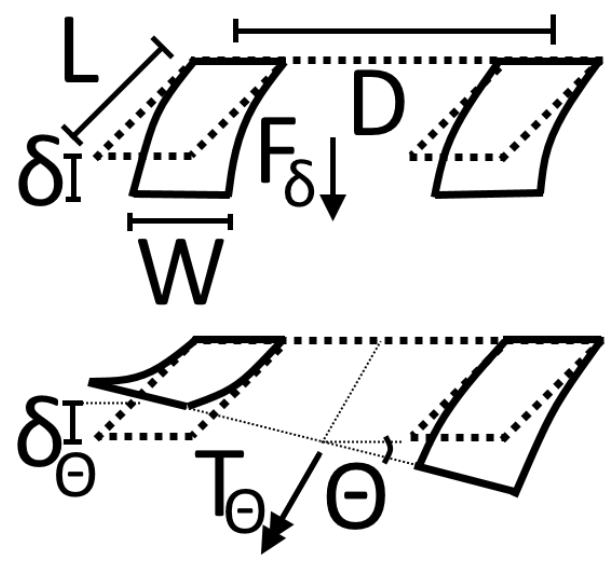

Figure 80: Double flexure diagram

Table 36: Double flexure variables

\begin{tabular}{lll}
\hline Variable & Name & Value \\
\hline $\mathrm{T}$ & Flexure Thickness [in.] & 0.025 \\
$\mathrm{~W}$ & Flexure Width [in.] & 0.625 \\
$\mathrm{~L}$ & Overhung Length [in.] & 1.18 \\
$\mathrm{D}$ & Flexure Spacing [in.] & 3.5 \\
$\mathrm{E}$ & Modulus of Elasticity, Steel [psi] & $30.0 \mathrm{E} 6$ \\
$\mathrm{G}$ & Shear Modulus, Steel [psi] & $11.5 \mathrm{E} 6$ \\
$\mathrm{~J}$ & Torsional Constant, Each [in. ${ }^{4}$ ] & Calculated \\
$\mathrm{I}$ & Area Moment of Inertia, Each [in. $\left.{ }^{4}\right]$ & Calculated \\
$\delta$ & Vertical Deflection [in.] & 0.125 \\
$\Theta$ & Twist Angle [degrees] & 3 \\
$\delta_{\Theta}$ & Vertical Deflection, Twist [in.] & Calculated \\
$\mathrm{F}_{\delta}$ & Force Required [lbf] & Calculated \\
$\mathrm{T}_{\Theta}$ & Torque Required [in. ${ }^{*}$ lbf] & Calculated \\
\hline
\end{tabular}

Analyzing a double flexure system is similar to a single flexure in vertical bending, but some creativity must be employed to approximate torsional forces.

The force required to deflect the double flexures vertically is calculated by simply multiplying the force required for each flexure by a factor of 2 . Using the equations derived in the previous calculation: 


$$
\begin{gathered}
F_{\delta}=2 *\left(\frac{3 \delta E\left(\frac{1}{12} W T^{3}\right)}{L^{3}}\right) \\
F_{\delta}=2 *\left(\frac{(.125 \text { in. })(30 E 6 \mathrm{psi})(0.625 \text { in. })(0.025 \text { in. })^{3}}{4(1.18 \mathrm{in} .)^{3}}\right) \\
F_{\delta}=11.1 \mathrm{lbf}
\end{gathered}
$$

To determine the torque required to twist a double flexure, assume that the twisting motion involves two discrete steps:

1. Both flexures twist the angle $\Theta$.

2. The free ends of the flexures are displaced vertically an amount $+/-\delta_{\Theta}$.

This is not exact, but it provides a reasonable approximation of the force. For the prototype vehicle, the flexures are designed to be adjusted during testing to provide the forces required. These calculations are simply a starting point.

First, twist a single flexure by the angle $\Theta$. Solve for the torque required using the previously derived equation.

$$
\begin{gathered}
T_{\Theta 1}=\frac{\Theta\left(\frac{1}{3} W T^{3}\right) G}{L} \\
T_{\Theta 1}=\frac{(3 \text { degrees })\left(\frac{2 \pi}{360}\right)\left(\frac{1}{3}(0.625 \text { in. })(0.025 \text { in. })^{3}\right)(11.5 E 6 \mathrm{psi})}{1.18 \mathrm{in} .} \\
T_{\Theta 1}=1.62 \mathrm{in.} * \mathrm{lbf}
\end{gathered}
$$

Next, determine the vertical distance that the centers of the free ends would be displaced above or below equilibrium using basic trigonometry.

$$
\begin{gathered}
\delta_{\Theta}=\frac{D}{2} * \sin \Theta \\
\delta_{\Theta}=\frac{3.5 \text { in. }}{2} * \sin (3 \text { degrees }) \\
\delta_{\Theta}=0.091 \mathrm{in} .
\end{gathered}
$$

Find the force required to deflect each flexure by $\delta_{\Theta}$. 


$$
\begin{gathered}
F_{\delta}=\frac{3 \delta_{\Theta} E\left(\frac{1}{12} W T^{3}\right)}{L^{3}} \\
F_{\delta}=\frac{(0.091 \mathrm{in} .)(30 E 6 \mathrm{psi})(0.625 \mathrm{in} .)(0.025 \mathrm{in} .)^{3}}{4(1.18 \mathrm{in} .)^{3}} \\
F_{\delta}=4.05 \mathrm{lbf}
\end{gathered}
$$

To represent this force as a moment, multiply it by the distance to the global rotational

axis.

$$
\begin{gathered}
T_{\Theta 2}=F_{\delta} * \frac{D}{2} \\
T_{\Theta 2}=4.05 \mathrm{lbf} * \frac{3.5 \mathrm{in} .}{2} \\
T_{\Theta 2}=7.09 \text { in. } * \mathrm{lbf}
\end{gathered}
$$

To determine the total torque or moment required to twist both flexures by an angle $\Theta$, add the two torques together and multiply the sum by a factor of two to account for both flexures. This is the final torque required.

$$
\begin{gathered}
T_{\Theta}=2\left(T_{\Theta 1}+T_{\Theta 2}\right) \\
T_{\Theta}=2(1.62 \text { in.*lbf }+7.09 \text { in.*lbf }) \\
T_{\Theta}=17.5 \text { in.*lbf }
\end{gathered}
$$




\section{B. FRICTION TEST REPORT}

Date: $4 / 13 / 17$

Objective

The objective of this experiment is to determine the coefficient of an array of potential tread backing materials in order to determine the best possible candidates for use on the wall climbing vehicle. Additionally, the coefficient of friction between PTFE sheet and neoprene belt material is tested in order to validate the assumed value used for vehicle model calculations.

Equipment

- Electronic force gauge (Next-Shine Portable Electronic Scale)

- $\quad 100 \mathrm{~g}, 200 \mathrm{~g}$, and 500g calibration weights

- Weight pull sled

- Backing material samples, cut into 1"x1" squares

- $\quad$ Adhesive-backed PTFE sheet

- Surface samples (bare sheetrock, neoprene belt)

Procedure

1. Lay the surface sample materials on a level, flat table. Use a dry cloth to remove any dust or fine particulates from the surface.

2. Weigh and record the sled weight. This will be added to the calibration weights to determine the total normal force applied.

3. Place the first sample to be tested onto the surface. 
4. Place the weight sled on top of the sample, and add the $100 \mathrm{~g}$ calibration weight above the sample.

5. Using the electronic force gauge, slowly pull straight and level from the attachment point on the sled, taking care not to pull up or down on the sled. Watch for the maximum readout value on the scale.

6. Record the highest value observed.

7. Repeat steps 2 through 5 until five trials have been completed with the same weight and same sample.

8. Repeat steps 2 through 6 for weight increments of $200 \mathrm{~g}$ and $500 \mathrm{~g}$, recording all necessary data

9. Repeat steps 2 through 7 for all materials to be tested.

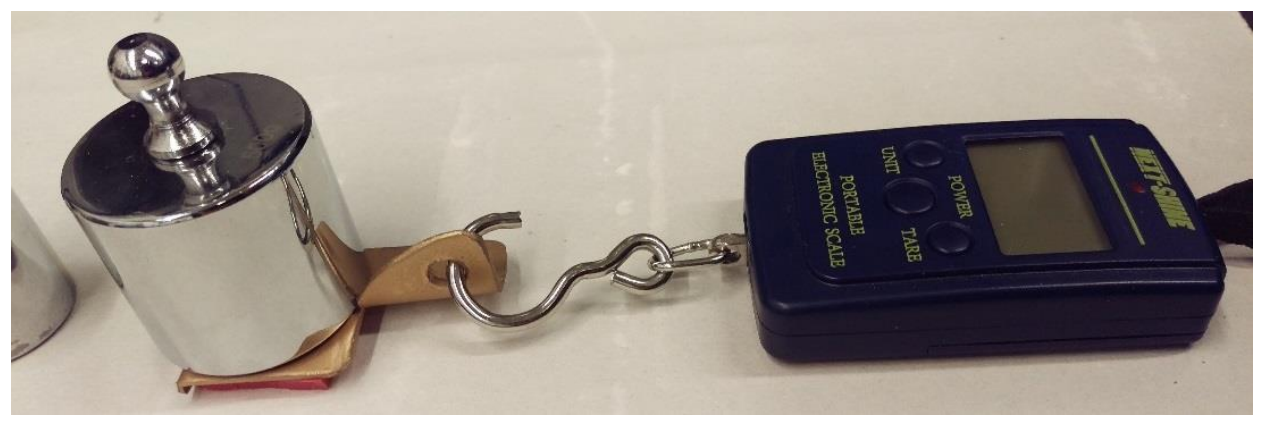

Figure 81: Friction test apparatus

Results

Ten sample backing materials were tested using the above procedures. Four of the sample materials had different surface finishes on the front and back sides, and as such were treated as different samples in the results. 

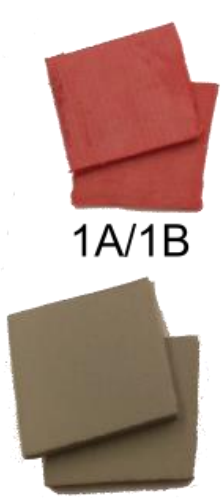

6

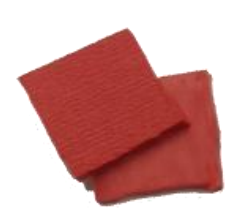

$2 \mathrm{~A} / 2 \mathrm{~B}$

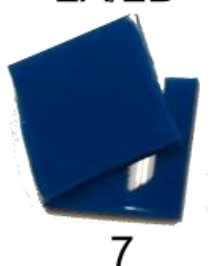

7

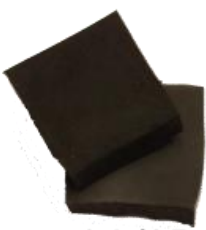

$3 \mathrm{~A} / 3 \mathrm{~B}$

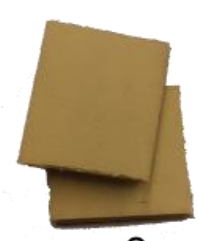

8
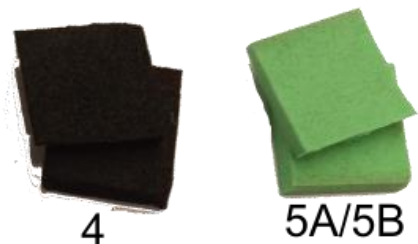

$5 \mathrm{~A} / 5 \mathrm{~B}$
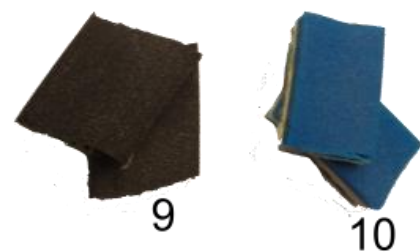

Figure 82: Test samples

The full data of the tests are printed at the end of the report. The critical results are summarized below.

Table 37: Friction test result summary

\begin{tabular}{|c|c|c|c|c|c|}
\hline No & Material & $\begin{array}{l}\text { Avg } \\
\mu\end{array}$ & $\begin{array}{l}\text { Qualitative } \\
\text { Hardness }\end{array}$ & $\begin{array}{l}\text { Vacuum } \\
\text { Suitable }\end{array}$ & Notes \\
\hline $1 \mathrm{~A}$ & $\begin{array}{l}\text { Thin Linatex, } \\
\text { Smooth }\end{array}$ & 0.68 & Firm & Yes & $\begin{array}{l}\text { Thin (0.067 in.) sheet, smooth side. Machinable. } \\
38 \text { D hardness confirmed by vendor. }\end{array}$ \\
\hline 1B & $\begin{array}{l}\text { Thin Linatex, } \\
\text { Rough }\end{array}$ & 0.89 & Firm & Yes & $\begin{array}{l}\text { Thin ( } 0.067 \text { in.) sheet, textured side. Machinable. } \\
38 \text { D hardness confirmed by vendor. }\end{array}$ \\
\hline $2 \mathrm{~A}$ & $\begin{array}{l}\text { Thick Linatex, } \\
\text { Smooth }\end{array}$ & 0.75 & Firm & Yes & $\begin{array}{l}\text { Thick ( } 0.175 \text { in.) sheet, smooth side. Machinable. } \\
38 \text { D hardness confirmed by vendor. }\end{array}$ \\
\hline 2B & $\begin{array}{l}\text { Thick Linatex, } \\
\text { Rough }\end{array}$ & 0.88 & Firm & Yes & $\begin{array}{l}\text { Thick ( } 0.175 \text { in. ) sheet, textured side. Machinable. } \\
38 \text { D hardness confirmed by vendor. }\end{array}$ \\
\hline $3 \mathrm{~A}$ & $\begin{array}{l}\text { Black Rubber, } \\
\text { Smooth }\end{array}$ & 0.58 & Soft & Yes & Very soft rubber, smooth side. Not machinable. \\
\hline $3 \mathrm{~B}$ & $\begin{array}{l}\text { Black Rubber, } \\
\text { Rough }\end{array}$ & 1.05 & Soft & Yes & Very soft rubber, textured side. Not machinable. \\
\hline 4 & Sponge Neoprene & 0.79 & Very Soft & Yes & $\begin{array}{l}\text { Low density, closed cell sponge material. Not } \\
\text { machinable. }\end{array}$ \\
\hline $5 \mathrm{~A}$ & $\begin{array}{l}\text { Sponge Urethane, } \\
\text { Smooth }\end{array}$ & 0.73 & Very Soft & No & $\begin{array}{l}\text { Medium density, mixed cell sponge material, } \\
\text { smooth/skinned side. }\end{array}$ \\
\hline $5 B$ & $\begin{array}{l}\text { Sponge Urethane, } \\
\text { Rough }\end{array}$ & 0.94 & Very Soft & No & $\begin{array}{l}\text { Medium density, mixed cell sponge material, } \\
\text { textured/open cell side. }\end{array}$ \\
\hline 6 & Neoprene & 0.89 & Soft & Yes & Medium density sponge material. 20-40 Duro. \\
\hline 7 & Urethane & 0.69 & Very Hard & Yes & Slick, smooth urethane. \\
\hline 8 & Natural Rubber & 0.93 & Firm & Yes & Tan natural rubber. 40 Duro. Machinable. \\
\hline 9 & Black Rubber 2 & 0.86 & Hard & Yes & Unknown black rubber \\
\hline 10 & Blue Rubber & 0.76 & Hard & Yes & Unknown blue rubber \\
\hline
\end{tabular}


In addition to the average friction coefficient over all weight levels, several qualitative characteristics regarding the foam were recorded as well. These included the materials' suitability to be used in vacuum systems. Open cell or mixed cell foam is not suitable for sealing purposes for obvious reasons. Additionally, relative hardness was recorded in order to judge the potential for sealing and conforming to surfaces that are not perfectly flat. Some materials were judged by the belt vendor to be not machinable to the level required by my design. A combination of factors will ultimately determine the material choice.

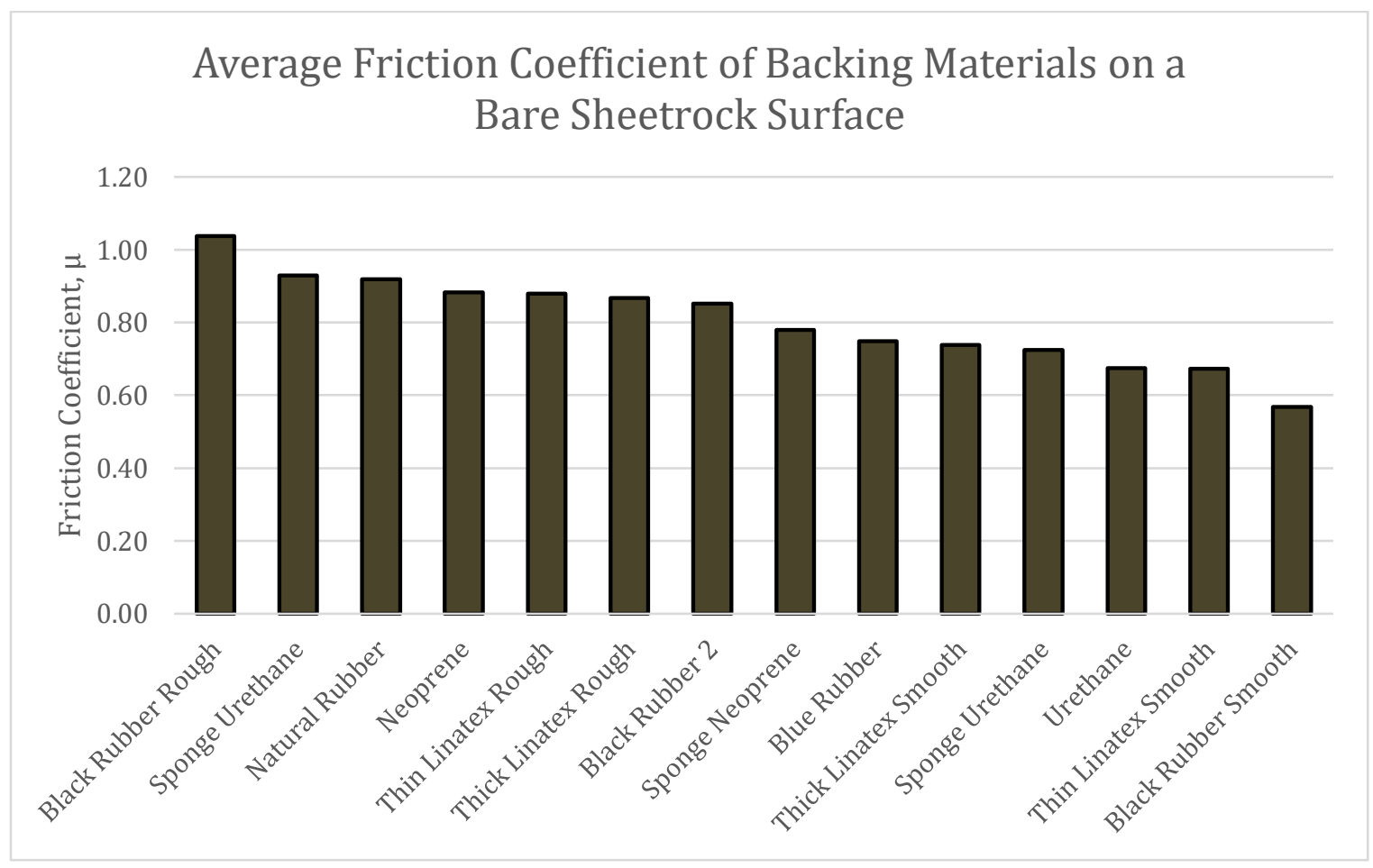

Figure 83: Average friction coefficients

As a last test, the adhesive-backed Teflon (PTFE) sheet was tested against a neoprene belt surface in order to simulate the sliding contact between the vacuum manifold and the sliding bed of the drive belts. The same procedure was used, including all three applied weights.

Table 38: PTFE friction results

\begin{tabular}{ll}
\hline Material & $\operatorname{Avg} \mu$ \\
\hline .030 in. PTFE on Neoprene Surface & 0.29 \\
\hline
\end{tabular}




\section{Conclusions}

The materials which demonstrated the best friction properties at all levels of normal force were the soft, black rubber (\#3B), sponge urethane (\#5B), natural rubber (\#8), neoprene (\#6) and the red Linatex (\#1B and \#2B). Machinability and vacuum concerns eliminate the black rubber, sponge urethane, and the neoprene foam, leaving the natural rubber and the Linatex as the top contenders. The softer surface of the Linatex compared to the natural rubber (38D Shore A vs 40D Shore A) as well as manufacturer literature indicating a preference to Linatex in high friction environments suggests that Linatex is the correct choice for this application.

Finally, the PTFE test provides a validated friction coefficient to utilize during design calculations. An enveloping friction coefficient of 0.4 will be applied for calculations. 
Raw Data

Tabulated below is the raw data from the friction test. Four trials of each weight level were tested, and the average of each set is calculated, as well as the overall average across all force levels.

Table 39: Friction test raw data

\begin{tabular}{|c|c|c|c|c|c|c|c|c|c|c|c|c|c|c|c|c|c|}
\hline \multicolumn{3}{|c|}{ Friction Test } & \multicolumn{3}{|c|}{ Normal Force [g] } & \multirow{2}{*}{$\begin{array}{r}120 \\
\text { Avg } \mu\end{array}$} & \multicolumn{4}{|c|}{ Normal Force [g] } & \multirow{2}{*}{$\begin{array}{c}220 \\
\operatorname{Avg} \mu\end{array}$} & \multicolumn{4}{|c|}{ Normal Force [g] } & \multirow{2}{*}{$\begin{array}{r}520 \\
\text { Avg } \mu\end{array}$} & \multirow{2}{*}{$\begin{array}{l}\text { Overall } \\
\text { Avg } \mu\end{array}$} \\
\hline Num & Material & T1 & $\mathrm{T} 2$ & T3 & T4 & & $\mathrm{T} 1$ & $\mathrm{~T} 2$ & T3 & T4 & & $\mathrm{T} 1$ & $\mathrm{~T} 2$ & T3 & T4 & & \\
\hline $3 B$ & Black Rubber Rough & 130 & 135 & 120 & 135 & 1.08 & 225 & 225 & 230 & 225 & 1.03 & 510 & 535 & 515 & 520 & 1.00 & 1.04 \\
\hline $5 B$ & Sponge Urethane & 115 & 115 & 110 & 110 & 0.94 & 210 & 195 & 215 & 205 & 0.94 & 460 & 475 & 480 & 480 & 0.91 & 0.93 \\
\hline 8 & Natural Rubber & 115 & 115 & 110 & 105 & 0.93 & 210 & 200 & 200 & 205 & 0.93 & 480 & 480 & 450 & 465 & 0.90 & 0.92 \\
\hline 6 & Neoprene & 100 & 115 & 105 & 100 & 0.88 & 190 & 205 & 200 & 200 & 0.90 & 460 & 460 & 445 & 445 & 0.87 & 0.88 \\
\hline $1 \mathrm{~B}$ & Thin Linatex Rough & 95 & 90 & 95 & 110 & 0.81 & 200 & 200 & 195 & 195 & 0.90 & 500 & 490 & 495 & 450 & 0.93 & 0.88 \\
\hline $2 B$ & Thick Linatex Rough & 105 & 95 & 115 & 100 & 0.86 & 175 & 190 & 190 & 195 & 0.85 & 475 & 460 & 455 & 450 & 0.88 & 0.87 \\
\hline 9 & Black Rubber 2 & 95 & 100 & 95 & 110 & 0.83 & 195 & 180 & 190 & 195 & 0.86 & 450 & 450 & 450 & 435 & 0.86 & 0.85 \\
\hline 4 & Sponge Neoprene & 75 & 95 & 90 & 95 & 0.74 & 175 & 190 & 185 & 180 & 0.83 & 405 & 415 & 405 & 375 & 0.77 & 0.78 \\
\hline 10 & Blue Rubber & 80 & 90 & 90 & 85 & 0.72 & 160 & 155 & 175 & 170 & 0.75 & 405 & 415 & 390 & 405 & 0.78 & 0.75 \\
\hline $2 \mathrm{~A}$ & Thick Linatex Smooth & 105 & 105 & 85 & 85 & 0.79 & 165 & 165 & 165 & 155 & 0.74 & 365 & 365 & 345 & 350 & 0.69 & 0.74 \\
\hline $5 \mathrm{~A}$ & Sponge Urethane & 75 & 90 & 90 & 80 & 0.70 & 195 & 160 & 165 & 155 & 0.77 & 390 & 370 & 360 & 360 & 0.71 & 0.73 \\
\hline 7 & Urethane & 55 & 60 & 70 & 80 & 0.55 & 135 & 170 & 170 & 150 & 0.71 & 415 & 345 & 410 & 420 & 0.76 & 0.68 \\
\hline $1 \mathrm{~A}$ & Thin Linatex Smooth & 75 & 80 & 80 & 70 & 0.64 & 150 & 155 & 155 & 145 & 0.69 & 375 & 345 & 390 & 340 & 0.70 & 0.67 \\
\hline $3 \mathrm{~A}$ & Black Rubber Smooth & 65 & 55 & 65 & 60 & 0.51 & 120 & 130 & 130 & 130 & 0.58 & 340 & 315 & 310 & 310 & 0.61 & 0.57 \\
\hline--- & Teflon on Neoprene & 30 & 40 & 35 & 30 & 0.28 & 60 & 65 & 45 & 60 & 0.26 & 180 & 180 & 170 & 175 & 0.34 & 0.29 \\
\hline
\end{tabular}

